\title{
Additive/multiplicative free subordination property and limiting eigenvectors of spiked additive deformations of Wigner matrices and spiked sample covariance matrices *
}

\author{
M. Capitaine ${ }^{\dagger}$
}

\begin{abstract}
When some eigenvalues of a spiked additive deformation of a Wigner matrix or a spiked multiplicative deformation of a Wishart matrix separate from the bulk, we study how the corresponding eigenvectors project onto those of the perturbation. We point out that the subordination function relative to the free (additive or multiplicative) convolution plays an important part in the asymptotic behavior.
\end{abstract}

\section{Introduction}

This paper lies in the lineage of recent works studying the influence of some perturbations on the asymptotic spectrum of classical random matrix models. Such questions come from Statistics (cf. [38) and appeared in the framework of empirical covariance matrices. In the pioneering work [11, J. Baik, G. Ben Arous and S. Péché dealt with random sample covariance matrices $\left(S_{N}\right)_{N}$ defined by

$$
S_{N}=\frac{1}{p} Y_{N} Y_{N}^{*} \text { with } Y=\Sigma_{N}^{\frac{1}{2}} B_{N}
$$

where $B_{N}$ is a $N \times p$ complex matrix such that the entries $\left(B_{N}\right)_{i j}$ are i.i.d centered standard Gaussian and $\Sigma_{N}$ is a deterministic positive $N \times N$ matrix having all but finitely many eigenvalues equal to one. This model can be seen as a multiplicative perturbation of the so-called white Wishart matrix for which $\Sigma_{N}=I_{N}$. Besides, the size of the samples $N$ and the size of the population $p=p_{N}$ are assumed of the same order (as $N \rightarrow \infty, N / p \rightarrow c>0$ ). The global limiting behavior of the spectrum of $S_{N}$ is not affected by such a matrix $\Sigma_{N}$.

\footnotetext{
* This work was partially supported by the Agence Nationale de la Recherche grant ANR08-BLAN-0311-03.

${ }^{\dagger}$ CNRS, Institut de Mathématiques de Toulouse, Equipe de Statistique et Probabilités, F-31062 Toulouse Cedex 09. E-mail: mireille.capitaine@math.univ-toulouse.fr
} 
Thus, the limiting spectral measure is the well-known Marchenko-Pastur law (42]) defined by

$$
\mu_{\mathrm{MP}, c}(d x)=\max \left\{1-\frac{1}{c}, 0\right\} \delta_{0}+f(x) \mathbf{1}_{\left[(1-\sqrt{c})^{2} ;(1+\sqrt{c})^{2}\right]}(x) d x
$$

with

$$
f(x)=\frac{\sqrt{\left(x-(1-\sqrt{c})^{2}\right)\left((1+\sqrt{c})^{2}-x\right)}}{2 \pi c x} .
$$

When $\Sigma_{N}=I_{N}$, the largest eigenvalue of $S_{N}$ converges towards the right hand point of the support of the Marchenko-Pastur law (see [31, 8, 58,). When $\Sigma_{N} \neq I_{N}$, in [11 the authors pointed out a striking phase transition phenomenon for the asymptotic behaviour of the largest eigenvalue of $S_{N}$ (at the convergence and fluctuations levels) according to the value of the largest eigenvalue(s) of $\Sigma_{N}$. They showed in particular that when the largest eigenvalue of $\Sigma_{N}$ is far from one, the largest eigenvalue of $S_{N}$ converges outside the limiting Marchenko-Pastur support. In [12], J. Baik and J. Silverstein extended the result of [1] on the convergence of the extremal eigenvalues of complex or real non necessarily Gaussian matrices $S_{N}$ under finite four moments assumptions on the distribution of the entries of $B_{N}$.

When $S_{N}$ is still defined by (1.1), but now the limiting spectral distribution of $\Sigma_{N}$ is some compactly supported measure $\nu$ on $[0 ;+\infty[$, under finite second moments assumptions on the distribution of the entries of $B_{N}$, the spectral distribution of $S_{N}$ converges almost surely towards a probability measure $\mu_{L S D}$ which only depends on $c$ and $\nu$; denoting by $g_{\mu_{L S D}}(z)=\int \frac{1}{z-x} d \mu_{L S D}(x)$ the Stieltjes transform of $\mu_{L S D}$, for $z \in \mathbb{C}^{+}, g_{\mu_{L S D}}(z)$ is the unique solution $Z$ in $\left\{Z \in \mathbb{C},-\frac{(1-c)}{z}-c Z \in \mathbb{C}^{+}\right\}$of the equation

$$
Z=\int \frac{1}{z-t(1-c+c z Z)} d \nu(t)
$$

(see 42, 4, 32, 40, 48, 54, 57]). Very recently R. Rao and J. Silverstein 47] and Z. D. Bai and J. Yao [9] dealt with such a model assuming moreover that $\Sigma_{N}$ has a finite number of eigenvalues fixed outside the support of $\nu$ called spikes (or converging outside the support of $\nu$ in [47]), whereas the distance between the other eigenvalues of $\Sigma_{N}$ and the support of $\nu$ uniformly goes to zero. (Note that the assumptions in [47] are a bit more general). Under finite four moments assumptions on the distribution of the entries of $B_{N}$, the authors characterized the spikes of $\Sigma_{N}$ that generate jumps of eigenvalues of $S_{N}$ and described the corresponding limiting points outside the support of the limiting spectral distribution $\mu_{L S D}$ of $S_{N}$.

Several authors considered an additive analogue of the above setting that is, the influence on the asymptotic spectrum of the addition of some Hermitian deterministic perturbation $A_{N}$ to the rescaled so-called Hermitian Wigner $N \times N$ matrix $W_{N}$. 
Recall that, according to Wigner's work [55, 56] and further results of different authors (see [3] for a review), provided the common distribution $\mu$ of the entries is centered with variance $\sigma^{2}$, the large $N$-limiting spectral distribution of the rescaled complex Wigner matrix $X_{N}=\frac{1}{\sqrt{N}} W_{N}$ is the semicircle distribution $\mu_{\sigma}$ whose density is given by

$$
\frac{d \mu_{\sigma}}{d x}(x)=\frac{1}{2 \pi \sigma^{2}} \sqrt{4 \sigma^{2}-x^{2}} \mathbf{1}_{[-2 \sigma, 2 \sigma]}(x) .
$$

Moreover, if the fourth moment of the measure $\mu$ is finite, the largest (resp. smallest) eigenvalue of $X_{N}$ converges almost surely towards the right (resp. left) endpoint $2 \sigma$ (resp. $-2 \sigma$ ) of the semicircular support (cf. [10] or Theorem 2.12 in 3 ).

Let $A_{N}$ be a deterministic Hermitian matrix such that the spectral measure of $A_{N}$ weakly converges to some probability measure $\nu$ and $\left\|A_{N}\right\|$ is uniformly bounded in $N$. When $N$ becomes large, free probability provides us a good understanding of the global behaviour of the spectrum of $M_{N}=X_{N}+A_{N}$ where $X_{N}$ is a rescaled complex Wigner matrix. Indeed, the spectral distribution of $M_{N}$ weakly converges to the free convolution $\mu_{\sigma} \boxplus \nu$ almost surely and in expectation ( $\mathrm{cf}$ 1, 43] and [51, 26] for pioneering works). We refer the reader to [53] for an introduction to free probability theory.

Dealing with small rank perturbation of a G.U.E matrix $W_{N}^{G}$, S. Péché pointed out an analogue phase transition phenomenon as in the sample covariance setting for the convergence and the fluctuations of the largest eigenvalue of $M_{N}^{G}=W_{N}^{G} / \sqrt{N}+A_{N}$ with respect to the largest eigenvalue $\theta$ (independent of $N$ ) of $A_{N}$ [45. These investigations imply that, if $\theta$ is far enough from zero $(\theta>\sigma)$, then the largest eigenvalue of $M_{N}^{G}$ jumps above the support $[-2 \sigma, 2 \sigma]$ of the limiting spectral measure and converges (in probability) towards $\rho_{\theta}=\theta+\frac{\sigma^{2}}{\theta}$. Note that Z. Füredi and J. Komlós already exhibited such a phenomenon in [30] dealing with non-centered symmetric matrices.

In [29], D. Féral and S. Péché proved that the results of [45] still hold for a nonnecessarily Gaussian Wigner Hermitian matrix $W_{N}$ with sub-Gaussian moments and in the particular case of a rank one perturbation matrix $A_{N}$ whose entries are all $\frac{\theta}{N}$ for some real number $\theta$. In [23], the authors considered a deterministic Hermitian matrix $A_{N}$ of arbitrary fixed finite rank $r$ and built from a family of $J$ fixed non-null real numbers $\theta_{1}>\cdots>\theta_{J}$ independent of $N$ and such that each $\theta_{j}$ is an eigenvalue of $A_{N}$ of fixed multiplicity $k_{j}$ (with $\sum_{j=1}^{J} k_{j}=r$ ). They dealt with general Wigner matrices associated to some symmetric measure satisfying a Poincaré inequality. They proved that eigenvalues of $A_{N}$ with absolute value strictly greater than $\sigma$ generate some eigenvalues of $M_{N}$ which converge to some limiting points outside the support of $\mu_{\sigma}$. In [24, the authors investigated the asymptotic behavior of the eigenvalues of generalized spiked perturbations of Wigner matrices associated to some symmetric measure satisfying a Poincaré inequality. In this paper, the perturbation matrix $A_{N}$ is a deterministic Hermitian matrix whose spectral measure converges to some probability measure $\nu$ with compact support and such that $A_{N}$ has a fixed number of fixed eigenvalues (spikes) outside the support of $\nu$, whereas the distance between the other 
eigenvalues and the support of $\nu$ uniformly goes to zero as $N$ goes to infinity. It is established that only a particular subset of the spikes will generate some eigenvalues of $M_{N}$ which will converge to some limiting points outside the support of the limiting spectral measure. The phenomenon is completely analogous to the one described in [47] and 9] in the sample covariance setting.

Now, one can wonder in the spiked deformed Wigner matrix setting as well as in the spiked sample covariance matrix setting, when some eigenvalues separate from the bulk, how the corresponding eigenvectors of the deformed model project onto those of the perturbation. There are some results concerning finite rank perturbations: 44 in the real Gaussian sample covariance matrix setting, and [15] dealing with finite rank additive or multiplicative perturbations of unitarily invariant matrices. For a general perturbation, up to our knowledge nothing has been done concerning eigenvectors of deformed Wigner matrices. Dealing with sample covariance matrices, S. Péché and O. Ledoit [46] introduced a tool to study the average behaviour of the eigenvectors but it seems that this did not allow them to focus on the eigenvectors associated with the eigenvalues that separate from the bulk.

As already said, the limiting spectral distribution of the deformed Wigner model is described by the free convolution of the respective limiting spectral distributions. Moreover, the authors explained in 24 that the phenomenon of the eigenvalues separating from the bulk can be fully described in terms of free probability involving the subordination function related to the free additive convolution of a semicircular distribution with the limiting spectral distribution of the perturbation. Actually, as we will show below, the analogue results in the sample covariance matrix setting can also be described in terms of free probability involving the subordination function related to the free multiplicative convolution of a Marchenko-Pastur distribution with the limiting spectral distribution of the perturbation. Moreover, as already noticed by P. Biane in 20, free probability again has something to tell us about eigenvectors of deformed matricial models. Indeed, in this paper, we are going to describe in the deformed Wigner matrix setting as well as in the sample covariance matrix one, how the eigenvectors of the deformed model associated to the eigenvalues that separate from the bulk project onto those associated to the spikes of the perturbation, pointing out that the subordination functions relative to the free additive or multiplicative convolution play an important part in this asymptotic behavior. Note that the proof is exactly the same in the additive and the multiplicative cases.

In the sample covariance matrix model as well as in the deformed Wigner model, the convergence of the eigenvalues that separate from the bulk is deduced from a striking exact separation phenomenon, roughly stating that to each gap in the spectrum of the deformed model there corresponds a gap in the spectrum of the perturbation, these gaps splitting in exactly the same way the corresponding spectrum. For general deformed models, that is, dealing with other matrices than Wigner matrices in the additive case or other matrices 
than white Wishart matrices in the multiplicative case, such an exact separation phenomenon is not expected in full generality. Nevertheless, we express all the results in this paper in terms of the free additive respectively multiplicative subordination functions since we conjecture that, for other deformed models than deformed Wigner matrices and sample covariance matrices, the limiting values of the eigenvalues that separate from the bulk as well as the limiting values of the orthogonal projection of the corresponding eigenvectors onto those associated to the spikes of the perturbation will be given by the same quantities provided one deals with the corresponding subordination functions relative to the limiting spectral distribution of the non-deformed model. By the way, note that one can check that the results of F. Benaych-Georges and R. N. Rao in [15, concerning finite rank multiplicative or additive perturbation of a unitarily invariant matrix, about the convergence of the extremal eigenvalues and of the projection of the corresponding eigenvectors onto those of the perturbation can be rewritten in terms of subordination functions as conjectured.

The paper is organized as follows. In Section 2, we introduce the additive and multiplicative deformed models we consider in this paper; we also introduce some basic notations that will be used throughout the paper. Section 3 is devoted to definitions and results concerning free convolutions and subordination functions, some of them being necessary to state our main result Theorem 4.2 in Section 4. Note that we present a common formulation for the additive and multiplicative deformed models and a common proof in Section 5 and Section 6 , postponing in Section 7 the technical results that need a specific study for each model. Finally, an Appendix gathers several tools that will be used in the paper.

\section{Models and Notations}

Let $\mu$ be a probability measure with variance $\sigma^{2}$ which satisfies a Poincaré inequality with constant $C_{P I}$ (the definition of such an inequality is recalled in the Appendix). Note that this condition implies that $\mu$ has moments of any order (see Corollary 3.2 and Proposition 1.10 in [41]). In this paper, we will deform the following classical matricial models.

- Normalized Wigner matrices $X_{N}^{W}=\frac{1}{\sqrt{N}} W_{N}$

such that $W_{N}$ is a $N \times N$ Wigner Hermitian matrix associated to the distribution $\mu$ :

$\left(W_{N}\right)_{i i}, \sqrt{2} \Re\left(\left(W_{N}\right)_{i j}\right)_{i<j}, \sqrt{2} \Im\left(\left(W_{N}\right)_{i j}\right)_{i<j}$ are i.i.d., with distribution $\mu$.

- Sample covariance matrices $X_{N}^{S}=\frac{1}{p} B_{N} B_{N}^{*}$

such that $B_{N}$ is a $N \times p$ matrix such that $\sqrt{2} \Re\left(\left(B_{N}\right)_{i j}\right)_{1 \leq i \leq N, 1 \leq j \leq p}$, $\sqrt{2} \Im\left(\left(B_{N}\right)_{i j}\right)_{1 \leq i \leq N, 1 \leq j \leq p}$ are i.i.d., with distribution $\mu$. We assume that $\frac{N}{p} \rightarrow c>0$ when $N$ goes to infinity. 
For Wigner matrices, we will assume moreover that $\mu$ is symmetric since we will use results of 24] where this assumption is needed.

We will deform these models by respectively addition and multiplication by a deterministic Hermitian perturbation matrix $A_{N}$; in the multiplicative perturbation case, $A_{N}$ will be assumed to be nonnegative definite. In both cases, we assume that:

\section{Assumption A:}

The eigenvalues $\gamma_{i}=\gamma_{i}(N)$ of $A_{N}$ are such that the spectral measure $\mu_{A_{N}}:=$ $\frac{1}{N} \sum_{i=1}^{N} \delta_{\gamma_{i}}$ weakly converges to some probability measure $\nu$ with compact support. We assume that there exists a fixed integer $r \geq 0$ (independent of $N$ ) such that $A_{N}$ has $N-r$ eigenvalues $\beta_{j}(N)$ satisfying

$$
\max _{1 \leq j \leq N-r} \operatorname{dist}\left(\beta_{j}(N), \operatorname{supp}(\nu)\right) \underset{N \rightarrow \infty}{\longrightarrow} 0,
$$

where $\operatorname{supp}(\nu)$ denotes the support of $\nu$.

We also assume that there are $J$ fixed real numbers $\theta_{1}>\ldots>\theta_{J}$ independent of $N$ which are outside the support of $\nu$ and such that each $\theta_{j}$ is an eigenvalue of $A_{N}$ with a fixed multiplicity $k_{j}$ (with $\sum_{j=1}^{J} k_{j}=r$ ). The $\theta_{j}$ 's will be called the spikes or the spiked eigenvalues of $A_{N}$. The set of the spikes of $A_{N}$ will be denoted by $\Theta$ :

$$
\Theta:=\left\{\theta_{1} ; \ldots, \theta_{J}\right\}
$$

In the sample covariance matrix setting we assume $\theta_{J}>0$.

We will consider simultaneously the two deformed models:

$$
\begin{aligned}
& M_{N}^{W}=X_{N}^{W}+A_{N}=\frac{1}{\sqrt{N}} W_{N}+A_{N}, \\
& M_{N}^{S}=A_{N}^{\frac{1}{2}} X_{N}^{S} A_{N}^{\frac{1}{2}}=\frac{1}{p} A_{N}^{\frac{1}{2}} B_{N} B_{N}^{*} A_{N}^{\frac{1}{2}} .
\end{aligned}
$$

When the approaches are the same for the two models we adopt the notation $M_{N}$ standing for both $M_{N}^{W}$ and $M_{N}^{S}$. When the studies are specific to one of the two models, we will use the superscripts.

Actually, we assume without loss of generality in the sample covariance setting that the variance of $\mu$ is 1 since it corresponds to considering the rescaled matrix $\frac{M_{N}^{S}}{\sigma^{2}}$.

Throughout this paper, we will use the following notations.

- $\mathbb{C}^{+}$will denote the complex upper half plane $\{z \in \mathbb{C}, \Im z>0\}$. Similarly, $\mathbb{C}^{-}$will stand for $\{z \in \mathbb{C}, \Im z<0\}$.

- For a function $f$ differentiable in some neighborhood of a point $x$ in $\mathbb{R}$, we will denote by $f^{\prime}(x)$ the derivative of $f$. 
- For a vector subspace $\mathcal{V}$ of $\mathbb{C}^{N}$, we will denote by $\mathcal{V}^{\perp}$ its orthogonal supplementary subspace and by $P_{\mathcal{V}}$ the orthogonal projection onto $\mathcal{V}$.

$-\langle$,$\rangle will denote the Hermitian inner product on \mathbb{C}^{N}$ defined by $\langle a, b\rangle=b^{*} a$ for any $a, b$ in $\mathbb{C}^{N}$.

- \|\|$_{2}$ will denote the Euclidean norm on $\mathbb{C}^{N}$.

- We will denote by $M_{m \times q}(\mathbb{C})$ the set of $m \times q$ matrices with complex entries. \| $\|$ will denote the operator norm and for any matrix $M,\|M\|_{2}=$ $\left\{\operatorname{Tr}\left(M M^{*}\right)\right\}^{\frac{1}{2}}$.

- For any matrix $M$ in $M_{N \times N}(\mathbb{C})$, we will denote its kernel by $\operatorname{Ker}(M)$.

- $E_{i j}$ in $M_{m \times q}(\mathbb{C})$ stands for the matrix such that $\left(E_{i j}\right)_{k l}=\delta_{i k} \delta_{j l}$.

- For any $N \times N$ Hermitian matrix $M$, we will denote by

$$
\lambda_{1}(M) \geq \ldots \geq \lambda_{N}(M)
$$

its ordered eigenvalues.

- For a probability measure $\tau$ on $\mathbb{R}$, we denote by $\operatorname{supp}(\tau)$ its support and by ${ }^{c} \operatorname{supp}(\tau)$ its complement in $\mathbb{R}$.

- For a probability measure $\tau$ on $\mathbb{R}$, we denote by $g_{\tau}$ its Stieltjes transform defined for $z \in \mathbb{C} \backslash \mathbb{R}$ by

$$
g_{\tau}(z)=\int_{\mathbb{R}} \frac{d \tau(x)}{z-x}
$$

- $G_{N}$ denotes the resolvent of $M_{N}$ and $g_{N}$ the mean of the Stieltjes transform of the spectral measure of $M_{N}$, that is,

$$
g_{N}(z)=\mathbb{E}\left(\operatorname{tr}_{N} G_{N}(z)\right), z \in \mathbb{C} \backslash \mathbb{R}
$$

where $\operatorname{tr}_{N}$ is the normalized trace: $\operatorname{tr}_{N}=\frac{1}{N} \operatorname{Tr}$.

When it is necessary to distinguish the deformed Wigner matrix setting and the sample covariance matrix one, we will specify the resolvent or the Stieltjes transform by using the corresponding superscript as follows: $G_{N}^{W}, g_{N}^{W}$ and $G_{N}^{S}, g_{N}^{S}$.

- $C, K$ denote nonnegative constants which may vary from line to line.

As already mentioned in the introduction, the assumptions on $W_{N}$ and $A_{N}$ ensure that they are asymptotically free, and then the spectral distribution of $M_{N}^{W}$ weakly converges to the free convolution $\mu_{\sigma} \boxplus \nu$ almost surely and in expectation (cf [1, 43] and [51, 26] for pioneering works).

Concerning the sample covariance matrix model $M_{N}^{S}$, as already noticed in the introduction, its limiting spectral measure only depends on $c$ and $\nu$. Note 
that when the entries of $B_{N}$ are Gaussian (that is, if $\mu$ is Gaussian) we can assume that $A_{N}$ is diagonal by the invariance under unitary conjugation of the distribution of $X_{N}^{S}$. Then, since according to Corollary 4.3 .8 in [36], $X_{N}^{S}$ and $A_{N}$ are asymptotically free, we can conclude that the limiting spectral distribution of $M_{N}^{S}$ is actually the free multiplicative convolution of the limiting spectral measure of $X_{N}^{S}$, that is, $\mu_{\mathrm{MP}, c}$, with $\nu$, denoted by $\mu_{\mathrm{MP}, c} \otimes \nu$.

Thus, free additive and multiplicative convolutions provide a good understanding of the limiting global behaviour of the spectrum of the above deformed models. Moreover, [15, 16, 24, show us that free probability can also allow to locate isolated eigenvalues of deformed matricial models. In particular, in 24], the authors point out that the subordination function relative to the free additive convolution provides a good understanding of the outliers of deformed Wigner matrices. We will see in this paper that the subordination function relative to the free (additive or multiplicative) convolution plays again an important part in the asymptotic behaviour of the eigenvectors relative to the outliers. We introduce in the following section some results concerning free convolution that will be fundamental later on.

\section{Free convolution}

Free convolutions appear as natural analogues of the classical convolutions in the context of free probability theory. Denote by $\mathcal{M}$ the set of probability measures supported on the real line and by $\mathcal{M}^{+}$the ones supported on $[0 ;+\infty[$. For $\mu$ and $\nu$ in $\mathcal{M}$ one defines the free additive convolution $\mu \boxplus \nu$ of $\mu$ and $\nu$ as the distribution of $X+Y$ where $X$ and $Y$ are free self adjoint random variables with distribution $\mu$ and $\nu$. For $\mu$ and $\nu$ in $\mathcal{M}^{+}$, the free multiplicative convolution $\mu \otimes \nu$ of $\mu$ and $\nu$ is the distribution of $X^{\frac{1}{2}} Y X^{\frac{1}{2}}$ where $X$ and $Y$ are free positive random variables with distribution $\mu$ and $\nu$. We refer the reader to [53] for an introduction to free probability theory and to [49, 50] and 17. for free convolutions. In this section, we recall the analytic approach developped in [49, 50, to calculate the free convolutions of measures, we present the important subordination property, and describe more deeply subordination functions relative to free additive convolution by a semi-circular distribution and free multiplicative convolution by a Marchenko-Pastur distribution. We also recall characterizations of the complement of the support of these convolutions.

\subsection{Additive Free convolution}

Let $\tau$ be a probability measure on $\mathbb{R}$. Its Stieltjes transform $g_{\tau}$ is analytic on the complex upper half-plane $\mathbb{C}^{+}$. There exists a domain

$$
D_{\alpha, \beta}=\{u+i v \in \mathbb{C},|u|<\alpha v, v>\beta\}
$$


on which $g_{\tau}$ is univalent. Let $K_{\tau}$ be its inverse function, defined on $g_{\tau}\left(D_{\alpha, \beta}\right)$, and

$$
R_{\tau}(z)=K_{\tau}(z)-\frac{1}{z}
$$

Given two probability measures $\tau$ and $\nu$, there exists a unique probability measure $\lambda$ such that

$$
R_{\lambda}=R_{\tau}+R_{\nu}
$$

on a domain where these functions are defined. The probability measure $\lambda$ is called the additive free convolution of $\tau$ and $\nu$ and denoted by $\tau \boxplus \nu$.

\subsubsection{Subordination property}

The free additive convolution of probability measures has an important property, called subordination, which can be stated as follows.

Proposition 3.1. let $\tau$ and $\nu$ be two probability measures on $\mathbb{R}$; there exists a unique analytic map $F^{(a)}: \mathbb{C}^{+} \rightarrow \mathbb{C}^{+}$such that

$$
\begin{gathered}
\forall z \in \mathbb{C}^{+}, \quad g_{\tau \boxplus \nu}(z)=g_{\nu}\left(F^{(a)}(z)\right), \\
F^{(a)}(\bar{z})=\overline{F^{(a)}(z)}, \Im F^{(a)}(z) \geq \Im z, \lim _{y \rightarrow+\infty} \frac{F^{(a)}(i y)}{i y}=1 .
\end{gathered}
$$

This phenomenon was first observed by D. Voiculescu under a genericity assumption in [52, and then proved in generality in [19] Theorem 3.1. Later, a new proof of this result was given in 14, using a fixed point theorem for analytic self-maps of the upper half-plane.

\subsubsection{Free convolution by a semicircular distribution}

In 18, P. Biane provides a deep study of the free additive convolution by a semicircular distribution. We first recall here some of his results that will be useful in our approach. Let $\nu$ be a probability measure on $\mathbb{R}$. When $\tau$ in (3.1)

is the semi-circular distribution $\mu_{\sigma}$, let us denote by $F_{\sigma, \nu}^{(a)}$ the subordination function. In 18, P. Biane introduces the set

$$
\Omega_{\sigma, \nu}:=\left\{u+i v \in \mathbb{C}^{+}, v>v_{\sigma, \nu}(u)\right\},
$$

where the function $v_{\sigma, \nu}: \mathbb{R} \rightarrow \mathbb{R}^{+}$is defined by

$$
v_{\sigma, \nu}(u)=\inf \left\{v \geq 0, \int_{\mathbb{R}} \frac{d \nu(x)}{(u-x)^{2}+v^{2}} \leq \frac{1}{\sigma^{2}}\right\} .
$$

The boundary of $\Omega_{\sigma, \nu}$ is the graph of the continuous function $v_{\sigma, \nu}$. P. Biane proves the following 
Proposition 3.2. [18] The map

$$
H_{\sigma, \nu}: z \longmapsto z+\sigma^{2} g_{\nu}(z)
$$

is a homeomorphism from $\overline{\Omega_{\sigma, \nu}}$ to $\mathbb{C}^{+} \cup \mathbb{R}$ which is conformal from $\Omega_{\sigma, \nu}$ onto $\mathbb{C}^{+} . F_{\sigma, \nu}^{(a)}:\left\{\begin{array}{l}\mathbb{C}^{+} \cup \mathbb{R} \rightarrow \overline{\Omega_{\sigma, \nu}} \\ z \rightarrow z-\sigma^{2} g_{\mu_{\sigma} \boxplus \nu}(z)\end{array} \quad\right.$ is the inverse function of $H_{\sigma, \nu}$.

Considering $H_{\sigma, \nu}$ as an analytic map defined in the whole upper half-plane $\mathbb{C}^{+}$, it can be easily seen that

$$
\Omega_{\sigma, \nu}=\left(H_{\sigma, \nu}\right)^{-1}\left(\mathbb{C}^{+}\right) .
$$

In the following, we will denote $H_{\sigma, \nu}$ by $H$ to simplify the writing.

Remark 3.1. Note that according to Proposition 3.2,

$$
F_{\sigma, \nu}^{(a)}(\mathbb{R})=\partial \Omega_{\sigma, \nu}=\left\{u+i v_{\sigma, \nu}(u), u \in \mathbb{R}\right\}
$$

so that we have the following equivalence

$$
u \in F_{\sigma, \nu}^{(a)}(\mathbb{R}) \cap \mathbb{R} \Longleftrightarrow v_{\sigma, \nu}(u)=0 .
$$

The following characterization of the elements of the complement of the support of $\nu$ which are in the image of $\mathbb{R}$ by $F_{\sigma, \nu}^{(a)}$ readily follows:

$$
u \in F_{\sigma, \nu}^{(a)}(\mathbb{R}) \cap \mathbb{R} \backslash \operatorname{supp}(\nu) \Longleftrightarrow u \in^{c} \operatorname{supp}(\nu), H^{\prime}(u) \geq 0 .
$$

In [18], P. Biane obtains a description of the support of $\mu_{\sigma} \boxplus \nu$ from which, when $\nu$ is a compactly supported probability measure, the authors deduce in 24] a characterization of the complement of the support of $\mu_{\sigma} \boxplus \nu$ involving the support of $\nu$ and $H$.

Proposition 3.3.

$$
x \in^{c} \operatorname{supp}\left(\mu_{\sigma} \boxplus \nu\right) \Leftrightarrow \exists u \in \mathcal{O}^{(a)} \text { such that } x=H(u)
$$

where $\mathcal{O}^{(a)}$ is the open set

$$
\begin{aligned}
\mathcal{O}^{(a)} & :=\left\{u \in^{c} \operatorname{supp}(\nu), H^{\prime}(u)>0\right\} \\
& =\left\{u \in^{c} \operatorname{supp}(\nu), \sigma^{2} \int \frac{1}{(u-t)^{2}} d \nu(t)<1\right\} .
\end{aligned}
$$

Remark 3.2. $\mathcal{O}^{(a)} \subset \partial \Omega_{\sigma, \nu}$

This readily follows from Remark 3.1

Remark 3.3. Note that if $u_{1}<u_{2}$ are in $\left\{u \in^{c} \operatorname{supp}(\nu), H^{\prime}(u) \geq 0\right\}$, one has $H\left(u_{1}\right) \leq H\left(u_{2}\right)$. Indeed, by Cauchy-Schwarz inequality, we have

$$
\begin{aligned}
H\left(u_{2}\right)-H\left(u_{1}\right) & =\left(u_{2}-u_{1}\right)\left[1-\sigma^{2} \int_{\mathbb{R}} \frac{d \nu(x)}{\left(u_{1}-x\right)\left(u_{2}-x\right)}\right] \\
& \geq\left(u_{2}-u_{1}\right)\left[1-\sigma^{2} \sqrt{\left(-g_{\nu}^{\prime}\left(u_{1}\right)\right)\left(-g_{\nu}^{\prime}\left(u_{2}\right)\right)}\right] \geq 0 .
\end{aligned}
$$




\subsection{Multiplicative free convolution}

Let $\tau \neq \delta_{0}$ be a probability measure on $[0 ;+\infty[$. Define the analytic function

$$
\Psi_{\tau}(z)=\int \frac{t z}{1-t z} d \tau(t)=\frac{1}{z} g_{\tau}\left(\frac{1}{z}\right)-1,
$$

for complex values of $z$ such that $\frac{1}{z}$ is not in the support of $\tau$. $\Psi_{\tau}$ determines uniquely the measure $\tau$ and it is univalent in the left half-plane $\{z \in \mathbb{C}, \Re z<0\}$. Then one may determine an analytic function $S_{\tau}$ such that

$$
\Psi_{\tau}\left[\frac{z}{z+1} S_{\tau}(z)\right]=z
$$

in some domain (which will contain at least some interval to the left of zero) and then $S_{\mu 凶 \nu}=S_{\mu} S_{\nu}$. (see [50]).

\subsubsection{Subordination property}

Free multiplicative convolution also presents a subordination phenomenon first proved in [19] (see also [14]).

Proposition 3.4. Let $\tau \neq \delta_{0}$ and $\nu \neq \delta_{0}$ be two probability measures on $\left[0 ;+\infty\left[\right.\right.$. There exists a unique analytic map $F_{\tau, \nu}^{(m)}$ defined on $\mathbb{C} \backslash[0 ;+\infty[$ such that

$$
\forall z \in \mathbb{C} \backslash\left[0 ;+\infty\left[, \Psi_{\nu \otimes_{\tau}}(z)=\Psi_{\nu}\left(F_{\tau, \nu}^{(m)}(z)\right)\right.\right.
$$

and

$$
\forall z \in \mathbb{C}^{+}, F_{\tau, \nu}^{(m)}(z) \in \mathbb{C}^{+}, F_{\tau, \nu}^{(m)}(\bar{z})=\overline{F_{\tau, \nu}^{(m)}(z)}, \arg \left(F_{\tau, \nu}^{(m)}(z)\right) \geq \arg (z) .
$$

\subsubsection{Multiplicative free convolution with a Marchenko-Pastur dis- tribution}

Let us determinate the subordination function relative to the free multiplication by a Marchenko-Pastur distribution. We can deduce from (1.3) that for any $z \in \mathbb{C}^{+}$,

$$
g_{\mu_{\mathrm{MP}, c} \otimes_{\nu}}(z)=\int \frac{1}{z-t\left(1-c+c z g_{\mu_{\mathrm{MP}, c} \otimes \nu}(z)\right)} d \nu(t)
$$

and then that $\forall z \in \mathbb{C} \backslash[0 ;+\infty[$,

$$
\Psi_{\mu_{\mathrm{MP}, c} \otimes_{\nu}}(z)=\Psi_{\nu}\left(z-c z+c g_{\mu_{\mathrm{MP}, c} \otimes_{\nu}}\left(\frac{1}{z}\right)\right) .
$$

Note that

$$
z-c z+c g_{\mu_{\mathrm{MP}, c} \otimes \nu}\left(\frac{1}{z}\right)=g_{\tau_{c, \nu}}\left(\frac{1}{z}\right)
$$

where $\tau_{c, \nu}$ is the limiting spectral distribution of $\frac{1}{p} B_{N}^{*} A_{N} B_{N}$. 
It is clear that $\forall z \in \mathbb{C}^{+}, g_{\tau_{c, \nu}}\left(\frac{1}{z}\right) \in \mathbb{C}^{+}, g_{\tau_{c, \nu}}\left(\frac{1}{\bar{z}}\right)=\overline{g_{\tau_{c, \nu}}\left(\frac{1}{z}\right)}$. Moreover, since, using (3.8), we have

$$
g_{\tau_{c, \nu}}\left(\frac{1}{z}\right)=z\left[(1-c)+c \int \frac{1}{1-t g_{\tau_{c, \nu}\left(\frac{1}{z}\right)}} d \nu(t)\right]
$$

it is easy to see that $\arg \left(g_{\tau_{c, \nu}}\left(\frac{1}{z}\right)\right) \geq \arg (z)$.

Therefore, denoting by $F_{c, \nu}^{(m)}$ the subordination function in (3.7) when $\tau=\mu_{\mathrm{MP}, c}$, we have that

$$
F_{c, \nu}^{(m)}(z)=z-c z+c g_{\mu_{\mathrm{MP}, c} \otimes \nu}\left(\frac{1}{z}\right)=g_{\tau_{c, \nu}}\left(\frac{1}{z}\right) .
$$

Now, we are going to present the characterization of the complement of the support of $\tau_{c, \nu}$ provided by Choi and Silverstein in [25]. Note that the supports of $\mu_{\mathrm{MP}, c} \otimes \nu$ and $\tau_{c, \nu}$ obviously coincide on $] 0 ;+\infty[$.

According to [7] p 113, for each $z \in \mathbb{C}^{+}, g_{\tau_{c, \nu}}(z)$ is the unique solution $Z$ in $\mathbb{C}^{-}$ of the equation

$$
Z=\frac{1}{z-c \int \frac{t}{1-t Z} d \nu(t)}
$$

so that

$$
z=\mathcal{Z}_{c, \nu}\left(g_{\tau_{c, \nu}}(z)\right)
$$

where

$$
\mathcal{Z}_{c, \nu}(x)=\frac{1}{x}+c \int \frac{t}{1-t x} d \nu(t) .
$$

In the following, we will denote $\mathcal{Z}_{c, \nu}$ by $\mathcal{Z}$ to simplify the writing.

Proposition 3.5. 25] If $u \in \in^{c} \operatorname{supp}\left(\tau_{c, \nu}\right)$, then $s=g_{\tau_{c, \nu}}(u)$ satisfies

1. $s \in \mathbb{R} \backslash\{0\}$,

2. $\frac{1}{s} \in^{c} \operatorname{supp}(\nu)$,

3. $\mathcal{Z}^{\prime}(s)<0$.

Conversely if s satisfies (1), (2) and (3), then $u=\mathcal{Z}(s) \in^{c} \operatorname{supp}\left(\tau_{c, \nu}\right)$.

In particular, letting $z$ converge towards any element $u$ of ${ }^{c} \operatorname{supp}\left(\tau_{c, \nu}\right)$ in (3.11) leads to

$$
u=\mathcal{Z}\left(g_{\tau_{c, \nu}}(u)\right)
$$

In [25], the authors proved also that $\lim _{z \rightarrow u} \quad g_{\tau_{c, \nu}}(z)=g_{\tau_{c, \nu}}(u)$ exists for any $u$ in $\mathbb{R} \backslash\{0\}$.

$$
z \in \mathbb{C}^{+}
$$

We include here for the convenience of the reader some basic facts that will be used later on. 
Remark 3.4. For any $u \in \mathbb{R} \backslash\{0\}$ such that $g_{\tau_{c, \nu}}(u) \in \mathbb{R} \backslash\{0\}$ and $\frac{1}{g_{\tau_{c, \nu}}(u)} \in^{c}$ $\operatorname{supp}(\nu)$, we have $\mathcal{Z}\left[g_{\tau_{c, \nu}}(u)\right]=u$.

This readily follows by letting $z$ goes to $u$ in (3.11).

Let us introduce the open set

$$
\begin{aligned}
\mathcal{O}^{(m)} & :=\left\{u \in^{c} \operatorname{supp}(\nu) \backslash\{0\}, \mathcal{Z}^{\prime}\left(\frac{1}{u}\right)<0\right\} \\
& =\left\{u \in^{c} \operatorname{supp}(\nu) \backslash\{0\}, c \int \frac{t^{2}}{(u-t)^{2}} d \nu(t)<1\right\} .
\end{aligned}
$$

Remark 3.5. For any $u$ in $\mathcal{O}^{(m)}, g_{\tau_{c, \nu}}\left[\mathcal{Z}\left(\frac{1}{u}\right)\right]=\frac{1}{u}$.

Let us prove Remark 3.5. According to Proposition 3.5. for any $u \in \mathcal{O}^{(m)}$, $\mathcal{Z}\left(\frac{1}{u}\right) \in^{c} \operatorname{supp}\left(\tau_{c, \nu}\right)$ and then according to the same Proposition 3.5, $\frac{1}{g_{\tau_{c, \nu}}\left[\mathcal{Z}\left(\frac{1}{u}\right)\right]}$ also belongs to $\mathcal{O}^{(m)}$. Now, for any $a \neq b$ in $\mathcal{O}^{(m)}$,

$$
\mathcal{Z}\left(\frac{1}{b}\right)-\mathcal{Z}\left(\frac{1}{a}\right)=(b-a)\left[1-c \int \frac{t^{2}}{(a-t)(b-t)} d \nu(t)\right] ;
$$

by Cauchy-Schwartz inequality,

$$
\begin{aligned}
\left|c \int \frac{t^{2}}{(a-t)(b-t)} d \nu(t)\right| & \left.\leq\left\{c \int \frac{t^{2}}{(a-t)^{2}} d \nu(t)\right\}^{\frac{1}{2}}\left\{c \int \frac{t^{2}}{(b-t)^{2}} d \nu(t)\right\}^{\frac{1}{2}} 3.16\right) \\
& <1 .
\end{aligned}
$$

Hence we can conclude that

$$
\text { for any } a \neq b \text { in } \mathcal{O}^{(m)}, \mathcal{Z}\left(\frac{1}{b}\right) \neq \mathcal{Z}\left(\frac{1}{a}\right) \text {. }
$$

Since using (3.13) we have $\mathcal{Z}\left[g_{\tau_{c, \nu}}\left[\mathcal{Z}\left(\frac{1}{u}\right)\right]\right]=\mathcal{Z}\left(\frac{1}{u}\right)$, we can then deduce that $g_{\tau_{c, \nu}}\left[\mathcal{Z}\left(\frac{1}{u}\right)\right]=\frac{1}{u}$.

Remark 3.6. Using (3.15) and (3.16), we have for any $a \leq b$ in the set $\left\{u \neq 0, u \in \in^{c} \operatorname{supp}(\nu), \mathcal{Z}^{\prime}\left(\frac{1}{u}\right) \leq 0\right\}$ that $\mathcal{Z}\left(\frac{1}{a}\right) \leq \mathcal{Z}\left(\frac{1}{b}\right)$.

Remark 3.7. For any $\left.u \in \mathcal{O}^{(m)} \cap\right] 0 ;+\infty\left[\right.$, we have $\mathcal{Z}\left(\frac{1}{u}\right)>0$.

Indeed, assume that $\mathcal{Z}\left(\frac{1}{u}\right) \leq 0$. According to Proposition $3.5 \mathcal{Z}\left(\frac{1}{u}\right) \in^{c} \operatorname{supp}\left(\tau_{c, \nu}\right)$; $\mathcal{Z}\left(\frac{1}{u}\right) \leq 0$ implies that $\mathcal{Z}\left(\frac{1}{u}\right)$ is on the left hand side of $\operatorname{supp}\left(\tau_{c, \nu}\right)$ and therefore that $g_{\tau_{c, \nu}}\left(\mathcal{Z}\left(\frac{1}{u}\right)\right) \leq 0$. This leads to a contradiction with Remark 3.5 saying that $g_{\tau_{c, \nu}}\left(\mathcal{Z}\left(\frac{1}{u}\right)\right)=\frac{1}{u}>0$.

Remark 3.8. For any $u \neq 0$ in ${ }^{c} \operatorname{supp}(\nu)$ such that $\mathcal{Z}^{\prime}\left(\frac{1}{u}\right)>0$, we have $\frac{1}{u} \notin g_{\tau_{c, \nu}}(\mathbb{R} \backslash\{0\})$. 
Indeed, let us assume that there exists $v \in \mathbb{R} \backslash\{0\}$ such that $\frac{1}{u}=g_{\tau_{c, \nu}}(v)$. According to Proposition 3.5, $v$ belongs to $\operatorname{supp}\left(\tau_{c, \nu}\right)$. Using Remark 3.4, since $g_{\tau_{c, \nu}}(v) \in \mathbb{R} \backslash\{0\}$ and $\frac{1}{g_{\tau_{c, \nu}}(v)} \in^{c} \operatorname{supp}(\nu)$, we have $\mathcal{Z}\left[g_{\tau_{c, \nu}}(v)\right]=v$. It follows that for any $y>0$,

$$
\begin{aligned}
1 & =\frac{\mathcal{Z}\left[g_{\tau_{c, \nu}}(v+i y)\right]-\mathcal{Z}\left[g_{\tau_{c, \nu}}(v)\right]}{i y} \\
& =\frac{\mathcal{Z}\left[g_{\tau_{c, \nu}}(v+i y)\right]-\mathcal{Z}\left[g_{\tau_{c, \nu}}(v)\right]}{g_{\tau_{c, \nu}}(v+i y)-g_{\tau_{c, \nu}}(v)} \times \frac{g_{\tau_{c, \nu}}(v+i y)-g_{\tau_{c, \nu}}(v)}{i y} .
\end{aligned}
$$

Since $g_{\tau_{c, \nu}}(v+i y)$ converges towards $g_{\tau_{c, \nu}}(v)=\frac{1}{u}$ and $\mathcal{Z}$ is holomorphic in a neighborhood of $g_{\tau_{c, \nu}}(v)$, letting $y$ tends to zero the first factor on the right hand side of (3.18) converges towards $\mathcal{Z}^{\prime}\left(g_{\tau_{c, \nu}}(v)\right)=\mathcal{Z}^{\prime}\left(\frac{1}{u}\right)>0$. This implies that $\Re\left[\frac{g_{\tau_{c, \nu}}(v+i y)-g_{\tau_{c, \nu}}(v)}{i y}\right]$ converges towards $\frac{1}{\mathcal{Z}^{\prime}\left(\frac{1}{u}\right)}>0$ when $y$ tends to zero. Now, for any $y>0$, we have

$$
\begin{aligned}
\Re\left[\frac{g_{\tau_{c, \nu}}(v+i y)-g_{\tau_{c, \nu}}(v)}{i y}\right] & =\frac{\Im\left[g_{\tau_{c, \nu}}(v+i y)-g_{\tau_{c, \nu}}(v)\right]}{y} \\
& =\frac{\Im g_{\tau_{c, \nu}}(v+i y)}{y}<0
\end{aligned}
$$

which leads to a contradiction.

\section{Main results}

As noticed in the previous section, we have the following characterization of the complement of the support of the limiting spectral distribution of $M_{N}^{W}$.

$$
x \in \in^{c} \operatorname{supp}\left(\mu_{\sigma} \boxplus \nu\right) \Leftrightarrow \exists u \in \mathcal{O}^{(a)} \text { such that } x=H(u) .
$$

Moreover, we can deduce, using Proposition 3.2 and Remark 3.2 that : $x \mapsto F_{\sigma, \nu}^{(a)}(x)$ is a bijection from ${ }^{c} \operatorname{supp}\left(\mu_{\sigma} \boxplus \nu\right)$ onto $\mathcal{O}^{(a)}$ with inverse $H$.

Since the supports of $\mu_{\mathrm{MP}, c} \otimes \nu$ and $\tau_{c, \nu}$ coincide on $] 0 ;+\infty[$, we can also deduce from the previous section the following characterization of the restriction to $\mathbb{R} \backslash\{0\}$ of the complement of the support of the limiting spectral distribution of $M_{N}^{S}$ :

$$
x \neq 0, x \in^{c} \operatorname{supp}\left(\mu_{\mathrm{MP}, c} \otimes \nu\right) \Leftrightarrow \exists u \in \mathcal{O}^{(m)}, \mathcal{Z}\left(\frac{1}{u}\right) \neq 0 \text {, such that } x=\mathcal{Z}\left(\frac{1}{u}\right) .
$$

Moreover, we can deduce from Remark 3.4 and Remark 3.5 that:

$x \mapsto F_{c, \nu}^{(m)}\left(\frac{1}{x}\right)\left(=g_{\tau_{c, \nu}}(x)\right)$ is a bijection from ${ }^{c} \operatorname{supp}\left(\mu_{\mathrm{MP}, c} \otimes \nu\right) \backslash\{0\}$ onto the set $\left\{\frac{1}{u}, u \in \mathcal{O}^{(m)}, \mathcal{Z}\left(\frac{1}{u}\right) \neq 0\right\}$ with inverse $\mathcal{Z}$. 
Note that the limiting mass at zero was studied in [25]:

$$
\mu_{\mathrm{MP}, c} \otimes \nu(0)=\left\{\begin{array}{l}
\nu(0) \text { if } c(1-\nu(0)) \leq 1, \\
1-\frac{1}{c} \text { if } c(1-\nu(0))>1 .
\end{array}\right.
$$

Actually, according to 24] (resp. 47, 9]), the spikes $\theta_{j}$ in $\Theta=\left\{\theta_{1} ; \ldots ; \theta_{J}\right\}$ of the perturbation matrix $A_{N}$ that will generate eigenvalues of $M_{N}^{W}$ (resp. $M_{N}^{S}$ ) which deviate from the bulk are exactly those belonging to $\mathcal{O}^{(a)}$ (resp. $\mathcal{O}^{(m)}$ ) and the corresponding limiting points outside the support of $\mu_{\sigma} \boxplus \nu$ (resp. $\tau_{c, \nu}$ ) will be given by $H\left(\theta_{j}\right)$ (resp. $\mathcal{Z}\left(\frac{1}{\theta_{j}}\right)$ ). Note that the results in [47, 9] are not formulated in that way since the authors do not deal with subordination function but as already mentioned we choose to express all the results using these functions $H$ and $\mathcal{Z}$ related to the subordination functions for further generalizations. Hence adopting the notations of the first column of the following array standing for both the corresponding elements of the second column (deformed Wigner matrix case) and the third column (sample covariance matrix case), we can present a common formulation of these results.

\begin{tabular}{|c|c|c|}
\hline$M_{N}$ & $M_{N}^{W}$ & $M_{N}^{S}$ \\
\hline$\mu_{\mathrm{LSD}}$ & $\mu_{\sigma} \boxplus \nu$ & $\mu_{\mathrm{MP}, c} \otimes \nu$ \\
\hline $\mathcal{O}$ & $\mathcal{O}^{(a)}$ & $\mathcal{O}^{(m)}$ \\
\hline$\Theta_{o}$ & $\Theta \cap \mathcal{O}^{(a)}=\left\{\theta_{i} \in \Theta, H^{\prime}\left(\theta_{j}\right)>0\right\}$ & $\Theta \cap \mathcal{O}^{(m)}=\left\{\theta_{i} \in \Theta, \mathcal{Z}^{\prime}\left(\frac{1}{\theta_{j}}\right)<0\right\}$ \\
\hline$\rho_{\theta_{j}}$ & $H\left(\theta_{j}\right)$ & $\mathcal{Z}\left(\frac{1}{\theta_{j}}\right)$ \\
\hline
\end{tabular}

Theorem 4.1. [47, 9, 24] Let $\theta_{j}$ be in $\Theta_{o}$ and denote by $n_{j-1}+1, \ldots, n_{j-1}+k_{j}$ the descending ranks of $\theta_{j}$ among the eigenvalues of $A_{N}$. Then the $k_{j}$ eigenvalues $\left(\lambda_{n_{j-1}+i}\left(M_{N}\right), 1 \leq i \leq k_{j}\right)$ converge almost surely outside the support of $\mu_{L S D}$ towards $\rho_{\theta_{j}}$. Moreover, these eigenvalues asymptotically separate from the rest of the spectrum since (with the conventions that $\lambda_{0}\left(M_{N}\right)=+\infty$ and $\left.\lambda_{N+1}\left(M_{N}\right)=-\infty\right)$ there exists $0<\delta_{0}$ such that almost surely for all large $N$,

$$
\lambda_{n_{j-1}}\left(M_{N}\right)>\rho_{\theta_{j}}+\delta_{0} \text { and } \lambda_{n_{j-1}+k_{j}+1}\left(M_{N}\right)<\rho_{\theta_{j}}-\delta_{0} .
$$

The aim of this paper is to study how the corresponding eigenvectors of the deformed model project onto those of the perturbation. Here is the main result of the paper still adopting the notations of the first column of the array (4.3) in order to present a unified approach.

Theorem 4.2. Let $\theta_{j}$ be in $\Theta_{o}$ and denote by $n_{j-1}+1, \ldots, n_{j-1}+k_{j}$ the descending ranks of $\theta_{j}$ among the eigenvalues of $A_{N}$. Let $\xi(j)$ be a normalized eigenvector of $M_{N}$ relative to one of the eigenvalues $\left(\lambda_{n_{j-1}+q}\left(M_{N}\right), 1 \leq q \leq k_{j}\right)$. Then, when $N$ goes to infinity,

(i) $\left\|P_{K e r\left(\theta_{j} I_{N}-A_{N}\right)} \xi(j)\right\|_{2}^{2} \stackrel{a . s}{\rightarrow} \tau\left(\theta_{j}\right)$ 
where

$$
\tau\left(\theta_{j}\right)=\left\{\begin{array}{l}
H^{\prime}\left(\theta_{j}\right) \text { if } M_{N}=M_{N}^{W}, \\
-\frac{\mathcal{Z}^{\prime}\left(\frac{1}{\theta_{j}}\right)}{\theta_{j} \mathcal{Z}\left(\frac{1}{\theta_{j}}\right)} \text { if } M_{N}=M_{N}^{S} .
\end{array}\right.
$$

Note that we have explicitly

$$
\begin{gathered}
H^{\prime}\left(\theta_{j}\right)=1-\sigma^{2} \int \frac{1}{\left(\theta_{j}-x\right)^{2}} d \nu(x), \\
-\frac{\mathcal{Z}^{\prime}\left(\frac{1}{\theta_{j}}\right)}{\theta_{j} \mathcal{Z}\left(\frac{1}{\theta_{j}}\right)}=\frac{1-c \int \frac{x^{2}}{\left(\theta_{j}-x\right)^{2}} d \nu(x)}{1+c \int \frac{x}{\left(\theta_{j}-x\right)} d \nu(x)} .
\end{gathered}
$$

(ii) for any $\theta_{l}$ in $\Theta \backslash\left\{\theta_{j}\right\}$,

$$
\left\|P_{K e r\left(\theta_{l} I_{N}-A_{N}\right)} \xi(j)\right\|_{2} \stackrel{a . s}{\rightarrow} 0 .
$$

Example: Let us consider the perturbation matrix

$$
A_{N}=\operatorname{diag}(2, \frac{3}{2}, 0, \underbrace{-1, \ldots,-1}_{\frac{N}{2}}, \underbrace{1, \ldots, 1}_{\frac{N}{2}-3}),
$$

whose limiting spectral distribution is $\nu=\frac{1}{2} \delta_{1}+\frac{1}{2} \delta_{-1}$. Thus, the set of the spikes of $A_{N}$ is $\Theta=\left\{2 ; \frac{3}{2} ; 0\right\}$. Let us consider the corresponding deformed Wigner model assuming moreover that $\sigma^{2}=\frac{1}{2}$. Then, $H(u)=u+\frac{1}{4} \frac{1}{(u-1)}+\frac{1}{4} \frac{1}{(u+1)}$. One can check that the support of $\mu_{\frac{1}{\sqrt{2}}} \boxplus \nu$ has two connected components which are symmetric with respect to zero. Since $H^{\prime}(2)=\frac{13}{18}>0$ and 2 is the largest eigenvalue of $A_{N}$, according to Theorem 4.1, when $N$ goes to infinity, the largest eigenvalue of the deformed Wigner model $M_{N}^{W}$ converges almost surely towards $H(2)=\frac{7}{3}$ (on the right hand side of the support of $\mu_{\frac{1}{\sqrt{2}}} \boxplus \nu$ ). Note that, since $H^{\prime}\left(\frac{3}{2}\right)<0$, the second largest eigenvalue of $M_{N}^{W}$ sticks to the bulk. Moreover, since $H^{\prime}(0)=\frac{1}{2}>0$ and the descending rank of 0 among the eigenvalues of $A_{N}$ is $\frac{N}{2}$, according to Theorem 4.1] when $N$ goes to infinity, $\lambda_{\frac{N}{2}}\left(M_{N}^{W}\right)$ converges almost surely towards $H(0)=0$ which is between the two connected components of the support of $\mu_{\frac{1}{\sqrt{2}}} \boxplus \nu$. Now, denote by $\left\{e_{1}, \ldots, e_{N}\right\}$ the canonical basis of $\mathbb{C}^{N}$. Since $e_{1}$ is an eigenvector relative to $2, e_{2}$ is an eigenvector relative to $\frac{3}{2}$ and $e_{3}$ is an eigenvector relative to 0 , according to Theorem 4.2, if $\xi$ denotes a normalized eigenvector associated to the largest eigenvalue of $M_{N}^{W}$, ${ }^{t} \xi=\left(\xi^{(1)}, \ldots, \xi^{(N)}\right)$, then $\left|\xi^{(1)}\right| \stackrel{a . s}{\rightarrow} \sqrt{H^{\prime}(2)}=\frac{\sqrt{13}}{3 \sqrt{2}}$ and, for $i=2,3,\left|\xi^{(i)}\right| \stackrel{a . s}{\rightarrow} 0$ when $N$ goes to infinity. Similarly, if $\xi$ denotes a normalized eigenvector associated to $\lambda_{\frac{N}{2}}\left(M_{N}^{W}\right),{ }^{t} \xi=\left(\xi^{(1)}, \ldots, \xi^{(N)}\right)$, then $\left|\xi^{(3)}\right| \stackrel{a . s}{\rightarrow} \sqrt{H^{\prime}(0)}=\frac{1}{\sqrt{2}}$ and, for $i=1,2,\left|\xi^{(i)}\right| \stackrel{a . s}{\rightarrow} 0$ when $N$ goes to infinity. 
Actually, in order to establish Theorem 4.2, we will first prove Proposition 4.1 below since when $k_{j} \neq 1$, the method used in this paper does not allow us to tackle directly the orthogonal projection of each eigenvector separately to prove (i).

Proposition 4.1. Let $\theta_{j}$ be in $\Theta_{o}$ and denote by $n_{j-1}+1, \ldots, n_{j-1}+k_{j}$ the descending ranks of $\theta_{j}$ among the eigenvalues of $A_{N}$. Denote by $\xi_{1}(j), \ldots, \xi_{k_{j}}(j)$ an orthonormal system of eigenvectors associated to $\left(\lambda_{n_{j-1}+n}\left(M_{N}\right), 1 \leq n \leq\right.$ $\left.k_{j}\right)$. Then, for any $\theta_{l}$ in $\Theta$, when $N$ goes to infinity,

$$
\sum_{n=1}^{k_{j}}\left\|P_{K e r\left(\theta_{l} I_{N}-A_{N}\right)} \xi_{n}(j)\right\|_{2}^{2} \stackrel{a . s}{\rightarrow} \delta_{l, j} k_{j} \tau\left(\theta_{j}\right)
$$

where $\tau\left(\theta_{j}\right)$ is defined by 4.5).

In Section 5 , we will explain how we can deduce (i) of Theorem 4.2 from Proposition 4.1] using a perturbation trick and ideas of [28].

\section{Reduction of the proof of (i) of Theorem 4.2 to the case of a spike with multiplicity one}

Note first that, dealing with a spike $\theta_{j}$ in $\Theta_{o}$ with multiplicity one, the statements of Theorem 4.2 and Proposition 4.1 are equivalent. Thus, in this section, we show how to deduce (i) of Theorem 4.2 dealing with a spike $\theta_{j}$ with multiplicity $k_{j} \neq 1$ from the hypothesis that (i) is true dealing with a spike with multiplicity one. We will need the following lemmas.

Lemma 5.1. Let $\mathcal{V}$ be a vector subspace of $\mathbb{C}^{N}$ with an orthonormal basis $V_{1}, \ldots, V_{k}$. Let $\alpha$ be in $\left[0 ;+\infty\left[\right.\right.$. For any $m=1, \ldots, k$, let $\alpha_{m}$ be in $[0 ;+\infty[$. Let $E$ be a vector subspace of $\mathbb{C}^{N}$ with an orthonormal basis $\xi_{1}, \ldots, \xi_{k}$. Then, there exists a sequence $a_{N} \geq 0$, depending on the $\left|\left\langle V_{i}, \xi_{n}\right\rangle\right|, \alpha_{m}, i, n, m \in\{1, \ldots, k\}^{3}$, such that, for any vector $u$ in the unit sphere of $\mathbb{C}^{N}$,

$$
\left|\left\|P_{\mathcal{V}} u\right\|_{2}^{2}-\alpha\right| \leq(2 k+\alpha)\left\|P_{E^{\perp}} u\right\|_{2}+\max _{m=1}^{k}\left|\alpha_{m}-\alpha\right|+a_{N},
$$

and, if, for any $m, n$ in $\{1, \ldots, k\}^{2}$,

$$
\left|\left\langle V_{m}, \xi_{n}\right\rangle\right|^{2} \rightarrow \delta_{m, n} \alpha_{m} \quad \text { when } N \text { goes to infinity, }
$$

then $a_{N}$ converges to zero when $N$ goes to infinity.

Proof: Throughout the proof, we will often use the following obvious inequalities without mentioning them:

for any vectors $u_{1}$ and $u_{2}$ in the unit sphere of $\mathbb{C}^{N},\left|\left\langle u_{1} u_{2}\right\rangle\right| \leq\left\|u_{1}\right\|_{2}\left\|u_{2}\right\|_{2}=1$, $\left|\left\langle P_{E} u_{1}, u_{2}\right\rangle\right| \leq\left\|P_{E} u_{1}\right\|_{2}\left\|u_{2}\right\|_{2} \leq\left\|u_{1}\right\|_{2}\left\|u_{2}\right\|_{2}=1$. 
We have for each $m$ in $\{1, \ldots, k\}$, for any vector $u$ in the unit sphere of $\mathbb{C}^{N}$,

$$
\begin{aligned}
\left|\left\langle u, V_{m}\right\rangle\right|^{2}-\left|\left\langle P_{E} u, V_{m}\right\rangle\right|^{2} \mid \\
=\quad\left|\left(\left|\left\langle u, V_{m}\right\rangle\right|-\left|\left\langle P_{E} u, V_{m}\right\rangle\right|\right)\right| \\
\quad \times\left(\left|\left\langle u, V_{m}\right\rangle\right|+\left|\left\langle P_{E} u, V_{m}\right\rangle\right|\right) \\
\leq \quad 2\left|\left(\left|\left\langle u, V_{m}\right\rangle\right|-\left|\left\langle P_{E} u, V_{m}\right\rangle\right|\right)\right| .
\end{aligned}
$$

From

$$
\left\langle u, V_{m}\right\rangle=\left\langle P_{E} u, V_{m}\right\rangle+\left\langle P_{E^{\perp}} u, V_{m}\right\rangle,
$$

it readily follows that

$$
||\left\langle u, V_{m}\right\rangle|-|\left\langle P_{E} u, V_{m}\right\rangle|| \leq\left|\left\langle P_{E^{\perp}} u, V_{m}\right\rangle\right| .
$$

(5.2) and (5.3) readily yield

$$
\begin{aligned}
\left.||\left\langle u, V_{m}\right\rangle\right|^{2}-\left|\left\langle P_{E} u, V_{m}\right\rangle\right|^{2} \mid & \leq 2\left|\left\langle P_{E^{\perp}} u, V_{m}\right\rangle\right| \\
& \leq 2\left\|P_{E^{\perp}} u\right\|_{2}\left\|V_{m}\right\|_{2} \\
& \leq 2\left\|P_{E^{\perp}} u\right\|_{2} .
\end{aligned}
$$

Now, we have that

$$
\begin{aligned}
\left\langle P_{E} u, V_{m}\right\rangle & =\left\langle u, \xi_{m}\right\rangle\left\langle\xi_{m}, V_{m}\right\rangle \\
+ & \sum_{\substack{n=1 \\
n \neq m}}^{k}\left\langle u, \xi_{n}\right\rangle\left\langle\xi_{n}, V_{m}\right\rangle \\
& =\left\langle u, \xi_{m}\right\rangle\left\langle\xi_{m}, V_{m}\right\rangle+\Delta_{m}(u)
\end{aligned}
$$

where

$$
\left|\Delta_{m}(u)\right| \leq \sum_{\substack{n=1 \\ n \neq m}}^{k}\left|\left\langle\xi_{n}, V_{m}\right\rangle\right|
$$

Then,

$$
\left|\left\langle P_{E} u, V_{m}\right\rangle\right|^{2}=\left|\left\langle u, \xi_{m}\right\rangle\right|^{2}\left|\left\langle\xi_{m}, V_{m}\right\rangle\right|^{2}+\nabla_{m}(u)
$$

with

$$
\left|\nabla_{m}(u)\right| \leq 2\left|\Delta_{m}(u)\right|+\left|\Delta_{m}(u)\right|^{2} .
$$

Thus,

$$
\begin{aligned}
\left|\left\langle P_{E} u, V_{m}\right\rangle\right|^{2}= & \alpha\left|\left\langle u, \xi_{m}\right\rangle\right|^{2} \\
& +\left(\alpha_{m}-\alpha\right)\left|\left\langle u, \xi_{m}\right\rangle\right|^{2} \\
& +\left(\left|\left\langle\xi_{m}, V_{m}\right\rangle\right|^{2}-\alpha_{m}\right)\left|\left\langle u, \xi_{m}\right\rangle\right|^{2} \\
& +\nabla_{m}(u) .
\end{aligned}
$$


Now, using that

$$
1=\|u\|_{2}^{2}=\sum_{m=1}^{k}\left|\left\langle u, \xi_{m}\right\rangle\right|^{2}+\left\|P_{E^{\perp}} u\right\|_{2}^{2},
$$

we have

$$
\begin{aligned}
\sum_{m=1}^{k}\left|\left\langle u, V_{m}\right\rangle\right|^{2}-\alpha= & \sum_{m=1}^{k}\left[\left|\left\langle u, V_{m}\right\rangle\right|^{2}-\alpha\left|\left\langle u, \xi_{m}\right\rangle\right|^{2}\right] \\
& -\alpha\left\|P_{E^{\perp}} u\right\|_{2}^{2}
\end{aligned}
$$

and then

$$
\begin{aligned}
\sum_{m=1}^{k}\left|\left\langle u, V_{m}\right\rangle\right|^{2}-\alpha= & \sum_{m=1}^{k}\left[\left|\left\langle u, V_{m}\right\rangle\right|^{2}-\left|\left\langle P_{E} u, V_{m}\right\rangle\right|^{2}\right] \\
& +\sum_{m=1}^{k}\left[\left|\left\langle P_{E} u, V_{m}\right\rangle\right|^{2}-\alpha\left|\left\langle u, \xi_{m}\right\rangle\right|^{2}\right] \\
& -\alpha\left\|P_{E^{\perp}} u\right\|_{2}^{2} .
\end{aligned}
$$

Using (5.9), (5.4), (5.8), (5.5) and (5.6), we immediately get that

$$
\begin{aligned}
\left.\left|\sum_{m=1}^{k}\right|\left\langle u, V_{m}\right\rangle\right|^{2}-\alpha \mid \leq & 2 k\left\|P_{E^{\perp}} u\right\|_{2} \\
& +\alpha\left\|P_{E^{\perp}} u\right\|_{2}^{2} \\
& +\max _{m=1}^{k}\left|\alpha_{m}-\alpha\right| \\
& +a_{N},
\end{aligned}
$$

with

$$
\begin{aligned}
a_{N}=\sum_{m=1}^{k} & \left.||\left\langle\xi_{m}, V_{m}\right\rangle\right|^{2}-\alpha_{m} \mid \\
+2 & \sum_{\substack{m, n=1 \\
n \neq m}}^{k}\left|\left\langle\xi_{n}, V_{m}\right\rangle\right|+\sum_{m=1}^{k}\left(\sum_{\substack{n=1 \\
n \neq m}}^{k}\left|\left\langle\xi_{n}, V_{m}\right\rangle\right|\right)^{2} .
\end{aligned}
$$

Now, if (5.1) is satisfied, that is, for each $m, n$ in $\{1, \ldots, k\}^{2}$, when $N$ goes to infinity,

$$
\left|\left\langle V_{m}, \xi_{n}\right\rangle\right|^{2} \rightarrow \delta_{m, n} \alpha_{m}
$$

it is clear that $a_{N}$ converges towards zero when $N$ goes to infinity. Lemma 5.1 follows. 
Lemma 5.2. Let $M_{N}$ be an Hermitian $N \times N$ matrix. Assume that there is a sequence $(n(N))_{N \geq 0}$ in $\mathbb{N}$ and a fixed positive integer number $k$, such that for any $l=1, \ldots, k, \lambda_{n(N)+l}\left(M_{N}\right)$ converges towards $\rho \in \mathbb{R}$ when $N$ goes to infinity and there exists $\delta_{0}>0$ such that, for all large $N$,

$$
\lambda_{n(N)}\left(M_{N}\right)>\rho+\delta_{0} \text { and } \lambda_{n(N)+k+1}\left(M_{N}\right)<\rho-\delta_{0}
$$

(with the conventions that $\lambda_{0}\left(M_{N}\right)=+\infty$ and $\lambda_{N+1}\left(M_{N}\right)=-\infty$ ). For any $0<\epsilon<\epsilon_{0}$, let $M_{N}(\epsilon)$ be an Hermitian $N \times N$ matrix. Assume that there exists $f_{\epsilon} \geq 0$, independent of $N$, decreasing to zero when $\epsilon$ decreases to zero, such that for all large $N$, for any $0<\epsilon<\epsilon_{0}$,

$$
\left\|M_{N}(\epsilon)-M\right\| \leq f_{\epsilon} .
$$

Let $0<\tilde{\epsilon}_{0}<\epsilon_{0}$ be such that $f_{\tilde{\epsilon}_{0}}<\frac{\delta_{0}}{4}$. Then for all large $N$, for any $0<\epsilon<\tilde{\epsilon}_{0}$, for any $l=1, \ldots, k$,

$$
\begin{gathered}
\left|\lambda_{n(N)+l}\left(M_{N}\right)(\epsilon)-\rho\right| \leq \frac{\delta_{0}}{2}, \\
\lambda_{n(N)}\left(M_{N}(\epsilon)\right)>\rho+\frac{\delta_{0}}{2} \text { and } \lambda_{n(N)+k+1}\left(M_{N}(\epsilon)\right)<\rho-\frac{\delta_{0}}{2},
\end{gathered}
$$

and for any normalized eigenvector $\xi$ of $M_{N}$ relative to the eigenvalue $\lambda_{n(N)+l}\left(M_{N}\right)$ for some $l$ in $\{1, \ldots, k\}$,

$$
\left\|P_{E(\epsilon) \perp} \xi\right\|_{2} \leq \frac{2}{\delta_{0}}\left\{f_{\epsilon}+\left|\lambda_{n(N)+l}\left(M_{N}\right)-\rho\right|\right\},
$$

where $E(\epsilon)$ denotes the vector subspace generated by the eigenvectors relative to the eigenvalues $\lambda_{n(N)+q}\left(M_{N}(\epsilon)\right), q=1, \ldots, k$.

Proof: According to Weyl inequalities (see Lemma 8.4 in the Appendix) and (5.12), for all large $N$, for all $0<\epsilon<\epsilon_{0}$,

$$
\begin{gathered}
\lambda_{n(N)+l}\left(M_{N}(\epsilon)\right) \leq \lambda_{n(N)+l}\left(M_{N}\right)+f_{\epsilon}, \text { for } l=1, \ldots, k+1, \\
\lambda_{n(N)+l}\left(M_{N}(\epsilon)\right) \geq \lambda_{n(N)+l}\left(M_{N}\right)-f_{\epsilon}, \text { for } l=0, \ldots, k .
\end{gathered}
$$

By assumptions of the lemma, for all large $N$, for any $l=1, \ldots, k$,

$$
\left|\lambda_{n(N)+l}\left(M_{N}\right)-\rho\right| \leq \frac{\delta_{0}}{4}
$$

and for all large $\mathrm{N}$,

$$
\lambda_{n(N)}\left(M_{N}\right)>\rho+\delta_{0} \text { and } \lambda_{n(N)+k+1}\left(M_{N}\right)<\rho-\delta_{0} .
$$

Hence, choosing $0<\tilde{\epsilon}_{0}<\epsilon_{0}$ such that $f_{\tilde{\epsilon}_{0}}<\frac{\delta_{0}}{4}$, (5.14) and (5.13) readily follow.

For all large $N$ and any $0<\epsilon<\tilde{\epsilon}_{0}$, let $\xi_{1}(\epsilon), \ldots, \xi_{k}(\epsilon)$ be an orthonormal basis of $E(\epsilon)$ such that there exists an $N \times N$ unitary matrix $V(\epsilon)$ whose $k$ first 
columns are $\xi_{1}(\epsilon), \ldots, \xi_{k}(\epsilon)$ and a $(N-k) \times(N-k)$ diagonal matrix $\Lambda_{2}(\epsilon)$ such that

$$
M_{N}(\epsilon)=V(\epsilon)\left(\begin{array}{cc}
\Lambda_{1}(\epsilon) & (0) \\
(0) & \Lambda_{2}(\epsilon)
\end{array}\right) V(\epsilon)^{*}
$$

where

$$
\Lambda_{1}(\epsilon)=\operatorname{diag}\left(\lambda_{n(N)+1}\left(M_{N}(\epsilon)\right), \ldots, \lambda_{n(N)+k}\left(M_{N}(\epsilon)\right)\right) .
$$

Let $\xi$ be a normalized eigenvector of $M_{N}$ relative to the eigenvalue $\lambda_{n(N)+l}\left(M_{N}\right)$ for some $l$ in $\{1, \ldots, k\}$. Let us set

$$
R(\epsilon):=\left(M_{N}(\epsilon)-\rho I_{N}\right) \xi .
$$

We have

$$
R(\epsilon)=V(\epsilon)\left(\begin{array}{cc}
\Lambda_{1}(\epsilon)-\rho I_{k} & (0) \\
(0) & \Lambda_{2}(\epsilon)-\rho I_{N-k}
\end{array}\right) V(\epsilon)^{*} \xi .
$$

Define the vector $v_{1}(\epsilon)$ in $\mathbb{C}^{k}$ and the vector $v_{2}(\epsilon)$ in $\mathbb{C}^{N-k}$ by setting

$$
V(\epsilon)^{*} \xi=\left(\begin{array}{c}
v_{1}(\epsilon) \\
v_{2}(\epsilon)
\end{array}\right)
$$

Note that

$$
\left\|v_{2}(\epsilon)\right\|_{2}=\left\|P_{E(\epsilon)^{\perp}} \xi\right\|_{2} .
$$

According to (5.14), for all large $N$, for any $0<\epsilon<\tilde{\epsilon}_{0}$, for all $i \notin\{n(N)+$ $1, \ldots, n(N)+k\},\left|\lambda_{i}\left(M_{N}(\epsilon)\right)-\rho\right|>\frac{\delta_{0}}{2}>0$ and therefore $\rho$ is not an eigenvalue of $\Lambda_{2}(\epsilon)$. Then

$$
v_{2}(\epsilon)=\left(\Lambda_{2}(\epsilon)-\rho I_{N-k}\right)^{-1}\left[V^{*}(\epsilon) R(\epsilon)\right]_{(N-k) \times 1}
$$

where $\left[V^{*}(\epsilon) R(\epsilon)\right]_{(N-k) \times 1}$ denotes the vector obtained from $V^{*}(\epsilon) R(\epsilon)$ after removing the first $k$ components. Hence

$$
\left\|v_{2}(\epsilon)\right\|_{2} \leq \frac{2}{\delta_{0}}\|R(\epsilon)\|_{2} .
$$

Now, we have

$$
\begin{aligned}
\|R(\epsilon)\|_{2} & =\left\|\left(M_{N}(\epsilon)-M_{N}+M_{N}-\rho I_{N}\right) \xi\right\|_{2} \\
& \leq\left\|M_{N}(\epsilon)-M_{N}\right\|+\left|\lambda_{n(N)+l}\left(M_{N}\right)-\rho\right|
\end{aligned}
$$

Lemma 5.2 readily follows from (5.15), (5.16), (5.17) and (5.12).

Let us define the continuous function $\tau$ on $\mathcal{O}^{(a)}$ respectively $\left.\mathcal{O}^{(m)} \cap\right] 0 ;+\infty[$ by setting

$$
\tau(x)=\left\{\begin{array}{l}
H^{\prime}(x) \text { if } M_{N}=M_{N}^{W}, \\
-\frac{\mathcal{Z}^{\prime}\left(\frac{1}{x}\right)}{x \mathcal{Z}\left(\frac{1}{x}\right)} \text { if } M_{N}=M_{N}^{S} .
\end{array}\right.
$$

Assume that $\theta_{j}$ in $\Theta_{o}$ is such that $k_{j} \neq 1$. Let us denote by $V_{1}(j), \ldots, V_{k_{j}}(j)$, an orthonormal system of eigenvectors of $A_{N}$ associated with $\theta_{j}$. There exists 
an $N \times N$ unitary matrix $U$ whose $k_{j}$ first columns are $V_{1}(j), \ldots, V_{k_{j}}(j)$ and a $\left(N-k_{j}\right) \times\left(N-k_{j}\right)$ Hermitian matrix $D$ such that

$$
A_{N}=U\left(\begin{array}{cc}
\theta_{j} I_{k_{j}} & (0) \\
(0) & D
\end{array}\right) U^{*} .
$$

Let us fix $\epsilon_{0}$ such that $0<\epsilon_{0}<\frac{1}{k_{j}} \min _{s=1}^{J} \operatorname{dist}\left(\theta_{s}\right.$, supp $\left.\nu \cup_{i \neq s} \theta_{i}\right)$ and $\left[\theta_{j} ; \theta_{j}+\right.$ $\left.k_{j} \epsilon_{0}\right] \subset \mathcal{O}$. For any $0<\epsilon<\epsilon_{0}$, let us consider

$$
M_{N}(\epsilon)=\left\{\begin{array}{l}
X_{N}+A_{N}(\epsilon) \text { if } X_{N}=X_{N}^{W} \\
A_{N}(\epsilon)^{\frac{1}{2}} X_{N} A_{N}(\epsilon)^{\frac{1}{2}} \text { if } X_{N}=X_{N}^{S}
\end{array}\right.
$$

where

$$
A_{N}(\epsilon)=U\left(\begin{array}{cc}
\operatorname{diag}\left(\theta_{j}+k_{j} \epsilon, \ldots, \theta_{j}+2 \epsilon, \theta_{j}+\epsilon\right) & (0) \\
(0) & D
\end{array}\right) U^{*} .
$$

Of course for any $0<\epsilon<\epsilon_{0}$, the limiting spectral distribution of $A_{N}(\epsilon)$, when $N$ goes to infinity, is the same as the limiting spectral distribution of $A_{N}$. Moreover, for all large $N$, the descending ranks $n_{j-1}+1, \ldots, n_{j-1}+k_{j}$ of $\theta_{j}$ among the eigenvalues of $A_{N}$ are the ranks of $\theta_{j}+k_{j} \epsilon, \ldots, \theta_{j}+2 \epsilon, \theta_{j}+\epsilon$ among the eigenvalues of $A_{N}(\epsilon)$. For each $m$ in $\left\{1, \ldots, k_{j}\right\}, V_{m}(j)$ is an eigenvector of $A_{N}(\epsilon)$ associated with the eigenvalue $\theta_{j}+\left(k_{j}-m+1\right) \epsilon$ which is of multiplicity one. Note that, since $A_{N}$ satisfies Assumption A, there exists a constant $C^{\prime}$ such that for any $0 \leq \epsilon<\epsilon_{0}, \sup _{N}\left\|A_{N}(\epsilon)\right\| \leq C^{\prime}$. It is easy to see that

$$
\left\|M_{N}(\epsilon)-M_{N}\right\| \leq\left\{\begin{array}{l}
\epsilon k_{j} \text { if } X_{N}=X_{N}^{W} \\
\left(C^{\prime}\right)^{\frac{1}{2}} \frac{k_{j}}{\sqrt{\theta_{j}}}\left\|X_{N}\right\| \epsilon \text { if } X_{N}=X_{N}^{S} .
\end{array}\right.
$$

According to Theorem 5.11 in [7], $\left\|X_{N}^{S}\right\|=c\left(1+\frac{1}{\sqrt{c}}\right)^{2}+o_{a . s, N}(1)$. Thus in both cases, there exists some constant $C_{1}$ such that a.s for all large $\mathrm{N}$, for any $0<\epsilon<\epsilon_{0}$,

$$
\left\|M_{N}(\epsilon)-M_{N}\right\| \leq C_{1} \epsilon .
$$

Let $\xi$ be a normalized eigenvector of $M_{N}$ relative to $\lambda_{n_{j-1}+q}\left(M_{N}\right)$ for some $q$ in $\left\{1, \ldots, k_{j}\right\}$. Let $0<\tilde{\epsilon}_{0}<\epsilon_{0}$ be chosen such that $C_{1} \tilde{\epsilon}_{0}<\frac{\delta_{0}}{4}$ where $\delta_{0}$ is defined in Theorem 4.1] Using Theorem 4.1 and (5.18), according to Lemma 5.2 almost surely for all large $N$, for any $0<\epsilon<\tilde{\epsilon}_{0}$, the set $\left\{\lambda_{n_{j-1}+n}\left(M_{N}(\epsilon)\right), n \in\right.$ $\left.\left\{1, \ldots, k_{j}\right\}\right\}$ is distinct from the set $\left\{\lambda_{i}\left(M_{N}(\epsilon)\right), i \notin\left\{n_{j-1}+1, \ldots, n_{j-1}+k_{j}\right\}\right\}$ and

$$
\left\|P_{E(\epsilon)^{\perp}} \xi\right\|_{2} \leq \frac{2}{\delta_{0}}\left\{C_{1} \epsilon+\left|\lambda_{n_{j-1}+q}\left(M_{N}\right)-\rho_{\theta_{j}}\right|\right\},
$$

where $E(\epsilon)$ denotes the vector subspace generated by the eigenvectors relative to the eigenvalues $\lambda_{n_{j-1}+n}\left(M_{N}(\epsilon)\right), n=1, \ldots, k_{j}$. Define

$$
\iota(\epsilon)=\frac{2\left(2 k_{j}+\tau\left(\theta_{j}\right)\right)}{\delta_{0}} C_{1} \epsilon+\max _{m=1}^{k_{j}}\left|\tau\left(\theta_{j}+m \epsilon\right)-\tau\left(\theta_{j}\right)\right| .
$$


For any $\zeta>0$, choose and fix $0<\epsilon=\epsilon_{1}<\tilde{\epsilon}_{0}$ such that

$$
0<\iota\left(\epsilon_{1}\right)<\frac{\zeta}{2}
$$

(using the continuity of the function $\tau$ at the point $\theta_{j}$ ).

Now, each $\theta_{j}+l \epsilon_{1}, l \in\left\{1, \ldots, k_{j}\right\}$, is a spike of $A_{N}\left(\epsilon_{1}\right)$ with multiplicity one. According to Theorem 4.1 for $n \in\left\{1, \ldots, k_{j}\right\}, \lambda_{n_{j-1}+n}\left(M_{N}\left(\epsilon_{1}\right)\right)$ asymptotically separates from the rest of the spectrum and converges almost surely towards $H\left(\theta_{j}+\left(k_{j}-n+1\right) \epsilon_{1}\right)$; moreover, if $\xi_{n}\left(\epsilon_{1}, j\right)$ denotes a normalized eigenvector associated to $\lambda_{n_{j-1}+n}\left(M_{N}\left(\epsilon_{1}\right)\right)$, Proposition 4.1 implies that

$$
\left|\left\langle V_{m}(j), \xi_{n}\left(\epsilon_{1}, j\right)\right\rangle\right|^{2} \stackrel{a . s}{\rightarrow} \delta_{m, n} \tau\left(\theta_{j}+\left(k_{j}-m+1\right) \epsilon_{1}\right) .
$$

According to Lemma 5.1, there exists a random variable $a_{N}\left(\epsilon_{1}\right) \geq 0$, converging almost surely to zero when $N$ goes to infinity, such that, almost surely, for all large $N$,

$$
\begin{aligned}
\left|\left\|P_{\operatorname{Ker}\left(\theta_{j} I_{N}-A_{N}\right)} \xi\right\|_{2}^{2}-\tau\left(\theta_{j}\right)\right| \leq & \left(2 k_{j}+\tau\left(\theta_{j}\right)\right)\left\|P_{E\left(\epsilon_{1}\right)^{\perp}} \xi\right\|_{2}+a_{N}\left(\epsilon_{1}\right) \\
& +\max _{m=1}^{k_{j}}\left|\tau\left(\theta_{j}+m \epsilon_{1}\right)-\tau\left(\theta_{j}\right)\right| .
\end{aligned}
$$

The last inequality, (5.19) and (5.20) readily yield that, almost surely, for all large $N$,

$$
\begin{aligned}
\left|\left\|P_{\operatorname{Ker}\left(\theta_{j} I_{N}-A_{N}\right)} \xi\right\|_{2}^{2}-\tau\left(\theta_{j}\right)\right| \leq & \frac{2}{\delta_{0}}\left(2 k_{j}+\tau\left(\theta_{j}\right)\right)\left|\lambda_{n_{j-1}+q}\left(M_{N}\right)-\rho_{\theta_{j}}\right| \\
& +a_{N}\left(\epsilon_{1}\right)+\iota\left(\epsilon_{1}\right) .
\end{aligned}
$$

Therefore, (5.21), the almost surely convergence of $\lambda_{n_{j-1}+q}\left(M_{N}\right)$ towards $\rho_{\theta_{j}}$ and of $a_{N}\left(\epsilon_{1}\right)$ towards zero imply that, almost surely, for all large $N$,

$$
\left|\left\|P_{\operatorname{Ker}\left(\theta_{j} I_{N}-A_{N}\right)} \xi\right\|_{2}^{2}-\tau\left(\theta_{j}\right)\right| \leq \zeta
$$

and the proof is complete.

\section{Proof of Proposition 4.1}

Since the proof of Proposition 4.1 is exactly the same for the deformed Wigner model and the sample covariance matrix, in order to present a unified approach of this proof, we adopt in this section the notations of the first column of the array (4.3) standing for both the corresponding elements of the second column (deformed Wigner matrix case) and the third column (sample covariance matrix case). We postpone in a later subsection the technical results that need a specific study for each model in order to not lose the thread of this common proof. 


\subsection{Restriction to the asymptotic behavior of some expec- tation $\mathbb{E} \operatorname{Tr}\left[h\left(M_{N}\right) f\left(A_{N}\right)\right]$}

The aim of this first step of the proof of Proposition 4.1 is to reduce the study of the asymptotic behaviour of $\sum_{n=1}^{k_{j}}\left\|P_{\operatorname{Ker}\left(\theta_{l} I_{N}-A_{N}\right)} \xi_{n}(j)\right\|_{2}^{2}$ to the one of the expectation $\mathbb{E} \operatorname{Tr}\left[h\left(M_{N}\right) f\left(A_{N}\right)\right]$ for some functions $f$ and $h$ respectively concentrated on a neighborhood of $\theta_{l}$ and $\rho_{\theta_{j}}$. We will use the convergence results on eigenvalues described in Theorem 4.1 above and concentration inequalities presented in the Appendix.

P. Biane already suggested in [20] to evaluate the moduli of the Hermitian inner products of the eigenvectors on test functions. Indeed, for any smooth function $h$ and $f$ on $\mathbb{R}$, denoted by $u_{1}, \ldots, u_{N}$ (resp. $\left.w_{1}, \ldots, w_{N}\right)$, the eigenvectors associated with $\lambda_{1}\left(A_{N}\right), \ldots, \lambda_{N}\left(A_{N}\right)$ (resp. $\left.\lambda_{1}\left(M_{N}\right), \ldots, \lambda_{N}\left(M_{N}\right)\right)$, one can easily check that

$$
\operatorname{Tr}\left[h\left(M_{N}\right) f\left(A_{N}\right)\right]=\sum_{k, i} h\left(\lambda_{k}\left(M_{N}\right)\right) f\left(\lambda_{i}\left(A_{N}\right)\right)\left|\left\langle u_{i}, w_{k}\right\rangle\right|^{2} .
$$

Thus, since $\theta_{l}$ on one hand and the $\lambda_{n_{j-1}+n}\left(M_{N}\right), n=1, \ldots, k_{j}$, on the other hand, asymptotically separate from the rest of the spectrum of respectively $A_{N}$ and $M_{N}$, a fit choice of $h$ and $f$ will allow the study of the restrictive sum $\sum_{n=1}^{k_{j}}\left\|P_{\operatorname{Ker}\left(\theta_{l} I_{N}-A_{N}\right)} \xi_{n}(j)\right\|_{2}^{2}$.

Let us fix

$$
0<\eta<\frac{1}{2} \min _{s=1}^{J} \operatorname{dist}\left(\theta_{s}, \operatorname{supp} \nu \cup_{i \neq s} \theta_{i}\right)
$$

and for any $l=1, \ldots, J$, choose $f_{\eta, l}$ in $\mathcal{C}^{\infty}(\mathbb{R}, \mathbb{R})$ with support in $\left[\theta_{l}-\eta, \theta_{l}+\eta\right]$ such that $f_{\eta, l}\left(\theta_{l}\right)=1$ and $0 \leq f_{\eta, l} \leq 1$.

For any $\theta_{i} \in \mathcal{O}^{(a)}$, according to Remark 3.2 , $\theta_{i}$ belongs to $\overline{\Omega_{\sigma, \nu}}$ and according to Proposition 3.2. $F_{\sigma, \nu}^{(a)}\left(\rho_{\theta_{i}}\right)=\theta_{i}$. For any $\left.\theta_{i} \in \mathcal{O}^{(m)} \cap\right] 0 ;+\infty[$, according to Remark 3.7, $\rho_{\theta_{i}}=\mathcal{Z}\left(\frac{1}{\theta_{i}}\right)>0$ and according to Remark 3.5] $g_{\tau_{c, \nu}}\left(\rho_{\theta_{i}}\right)=\frac{1}{\theta_{i}}$ so that $\frac{1}{F_{c, \nu}^{(m)}\left(\frac{1}{\rho_{\theta_{i}}}\right)}=\theta_{i}$.

According to Theorem 4.1 there exists $\delta_{0}>0$ such that almost surely for all large $\mathrm{N}$, for all $\theta_{j}$ in $\Theta_{o}$,

$$
\lambda_{n_{j-1}}\left(M_{N}\right)>\rho_{\theta_{j}}+\delta_{0} \text { and } \lambda_{n_{j-1}+k_{j}+1}\left(M_{N}\right)<\rho_{\theta_{j}}-\delta_{0} .
$$

Let us fix

$$
0<\delta<\frac{1}{3} \min \left\{\delta_{0}, \operatorname{dist}\left(\rho_{\theta_{s}}, \mathcal{S}\right),\left|\rho_{\theta_{s}}-\rho_{\theta_{t}}\right|, s \neq t,\left(\theta_{s}, \theta_{t}\right) \in \Theta_{o}^{2}\right\},
$$

where

$$
\mathcal{S}=\left\{\begin{array}{l}
\operatorname{supp}(\mu \boxplus \nu) \text { if } M_{N}=M_{N}^{W} \\
\operatorname{supp}\left(\tau_{c, \nu}\right) \cup\{0\} \text { if } M_{N}=M_{N}^{S}
\end{array} .\right.
$$


For any $j$ such that $\theta_{j} \in \Theta_{o}$, choose $h_{\delta, j}$ in $\mathcal{C}^{\infty}(\mathbb{R}, \mathbb{R})$ with support in $\left[\rho_{\theta_{j}}-\right.$ $\left.\delta, \rho_{\theta_{j}}+\delta\right]$ such that $h_{\delta, j} \equiv 1$ on $\left[\rho_{\theta_{j}}-\frac{\delta}{2}, \rho_{\theta_{j}}+\frac{\delta}{2}\right]$ and $0 \leq h_{\delta, j} \leq 1$.

Lemma 6.1. When $N$ goes to infinity

$$
\operatorname{Tr}\left[h_{\delta, j}\left(M_{N}\right) f_{\eta, l}\left(A_{N}\right)\right]-\sum_{n=1}^{k_{j}}\left\|P_{K e r\left(\theta_{l} I_{N}-A_{N}\right)} \xi_{n}(j)\right\|_{2}^{2} \stackrel{a . s}{\rightarrow} 0 .
$$

Proof: According to Theorem 4.1 there exists some set $\Omega$ of probability one such that on $\Omega$, for all large $N, \forall i=1, \ldots, k_{j}$,

$$
\begin{gathered}
\left|\lambda_{n_{j-1}+i}\left(M_{N}\right)-\rho_{\theta_{j}}\right|<\frac{\delta}{2} \\
\lambda_{n_{j-1}}\left(M_{N}\right) \geq \rho_{\theta_{j}}+\delta, \lambda_{n_{j-1}+k_{j}+1}\left(M_{N}\right) \leq \rho_{\theta_{j}}-\delta .
\end{gathered}
$$

Using also the assumption (2.1) on the $\beta_{i}(N)$ 's, we have that on $\Omega$, for all large $N$,

$$
\sum_{n=1}^{k_{j}}\left\|P_{\operatorname{Ker}\left(\theta_{l} I_{N}-A_{N}\right)} \xi_{n}(j)\right\|_{2}^{2}=\operatorname{Tr}\left[h_{\delta, j}\left(M_{N}\right) f_{\eta, l}\left(A_{N}\right)\right] .
$$

Hence, Lemma 6.1 follows.

Now, according to Lemma 8.1, Remark8.1 and Lemma 7.1, the random variables $F_{N}^{W}=\operatorname{Tr}\left[h_{\delta, j}\left(M_{N}^{W}\right) f_{\eta, l}\left(A_{N}\right)\right]$ and $F_{N}^{S}=\operatorname{Tr}\left[h_{\delta, j}\left(M_{N}^{S}\right) f_{\eta, l}\left(A_{N}\right)\right]$ satisfy respectively the following concentration inequalities

$$
\begin{aligned}
& \forall \epsilon>0, \mathbb{P}\left(\left|F_{N}^{W}-\mathbb{E}\left(F_{N}^{W}\right)\right|>\epsilon\right) \leq K_{1} \exp \left(-\frac{\epsilon \sqrt{N}}{K_{2} \sqrt{C_{P I} k_{l}}\left\|h_{\delta, j}\right\|_{L i p}}\right) . \\
& \forall \epsilon>0, \mathbb{P}\left(\left|F_{N}^{S}-\mathbb{E}\left(F_{N}^{S}\right)\right|>\epsilon\right) \leq K_{1} \exp \left(-\frac{\epsilon \sqrt{p}}{K_{2} \sqrt{C C_{P I} k_{l}}\left\|\tilde{h}_{\delta, j}\right\|_{L i p}}\right) .
\end{aligned}
$$

(The constants have been introduced in Lemma 8.1 and Lemma 7.1). By BorelCantelli Lemma, we can readily deduce the following lemma.

\section{Lemma 6.2.}

$$
\operatorname{Tr}\left[h_{\delta, j}\left(M_{N}\right) f_{\eta, l}\left(A_{N}\right)\right]-\mathbb{E}\left[\operatorname{Tr}\left[h_{\delta, j}\left(M_{N}\right) f_{\eta, l}\left(A_{N}\right)\right]\right] \stackrel{a . s}{\rightarrow} 0 .
$$

Lemma 6.1 and Lemma 6.2 allow us to conclude this first step of the proof by the following result.

Proposition 6.1. For any $\theta_{j}$ in $\Theta_{o}$ and any $\theta_{l}$ in $\Theta$, when $N$ goes to infinity

$$
\sum_{n=1}^{k_{j}}\left\|P_{K e r\left(\theta_{l} I_{N}-A_{N}\right)} \xi_{n}(j)\right\|_{2}^{2}-\mathbb{E}\left[\operatorname{Tr}\left[h_{\delta, j}\left(M_{N}\right) f_{\eta, l}\left(A_{N}\right)\right]\right] \stackrel{a . s}{\rightarrow} 0 .
$$




\subsection{Making use of estimations of the resolvent}

The basic idea of this second step of the proof of Proposition 4.1 is to approximate the function $h_{\delta, j}$ by its convolution by the Poisson Kernel in order to exhibit the resolvent of the deformed model and then use sharp estimations on this resolvent.

Lemma 6.3. For any continuous function $h$ with compact support and any bounded continuous function $\phi$,

$$
\mathbb{E}\left[\operatorname{Tr}\left[h\left(M_{N}\right) \phi\left(A_{N}\right)\right]\right]=-\lim _{y \rightarrow 0^{+}} \frac{1}{\pi} \Im \int \mathbb{E}\left(\operatorname{Tr}\left[G_{N}(t+i y) \phi\left(A_{N}\right)\right]\right) h(t) d t
$$

where $G_{N}(z)=\left(z I_{N}-M_{N}\right)^{-1}$.

Proof Let us denote by $P_{y}$ the Poisson kernel

$$
P_{y}(x)=\frac{y}{\pi\left(x^{2}+y^{2}\right)}, x \in \mathbb{R}, y>0 .
$$

We have

$$
\begin{aligned}
h(x) & =\lim _{y \rightarrow 0^{+}} h * P_{y}(x) \\
& =\lim _{y \rightarrow 0^{+}} \frac{1}{\pi} \int \frac{y h(t)}{(x-t)^{2}+y^{2}} d t \\
& =\lim _{y \rightarrow 0^{+}} \frac{1}{\pi} \int \Im \frac{h(t)}{x-i y-t} d t .
\end{aligned}
$$

Thus, for any fixed $N$,

$$
h\left(M_{N}\right)=-\lim _{y \rightarrow 0^{+}} \frac{1}{\pi} \int \Im G_{N}(t+i y) h(t) d t,
$$

and since $\left\|h * P_{y}\right\|_{\infty} \leq\|h\|_{\infty}$ we have $\left\|\int \Im G_{N}(t+i y) h(t) d t\right\| \leq\|h\|_{\infty}$. Then, the result readily follows by dominated convergence Theorem and Fubini's Theorem.

Let $U$ be a unitary matrix and

$$
D=\operatorname{diag}\left(\gamma_{1}, \ldots, \gamma_{N}\right)
$$

such that

$$
A_{N}=U^{*} D U
$$

Let $G$ stand for $G_{N}$ and $g$ stand for $g_{\mu_{\mathrm{LSD}}}$. Consider $\tilde{G}=U G U^{*}$. For any continuous function $\phi$,

$$
\operatorname{Tr}\left[G_{M_{N}}(z) \phi\left(A_{N}\right)\right]=\sum_{k=1}^{N} \phi\left(\gamma_{k}\right) \tilde{G}_{k k}(z) .
$$

The following result is fundamental in our approach. 
Proposition 6.2. There is a polynomial $P$ with nonnegative coefficients, a sequence $a_{N}$ of nonnegative real numbers converging to zero when $N$ goes to infinity and some nonnegative integer number $\alpha$, such that for any $k$ in $\{1, \ldots, N\}$, for all $z \in \mathbb{C} \backslash \mathbb{R}$,

$$
\mathbb{E}\left(\tilde{G}_{k k}(z)\right)=\tilde{\Phi}_{k}(z)+\Delta_{k, N}(z),
$$

with

$$
\left|\Delta_{k, N}(z)\right| \leq(1+|z|)^{\alpha} P\left(|\Im z|^{-1}\right) a_{N}
$$

where

$$
\tilde{\Phi}_{k}(z)= \begin{cases}\frac{1}{z-\sigma^{2} g(z)-\gamma_{k}}=\frac{1}{F_{\sigma, \nu}^{(a)}(z)-\gamma_{k}} \quad & \text { if } M_{N}=M_{N}^{W}, \\ \frac{1}{z-\gamma_{k}(1-c+c z g(z))}=\frac{1}{z\left(1-\gamma_{k} F_{c, \nu}^{(m)}\left(\frac{1}{z}\right)\right)} & \text { if } M_{N}=M_{N}^{S} .\end{cases}
$$

Note that $\tilde{\Phi}_{k}(z)$ is well defined for any $z \in \mathbb{C} \backslash \mathbb{R}$ since

$$
\begin{gathered}
\left|\Im\left[z-\sigma^{2} g(z)-\gamma_{k}\right]\right|=|\Im(z)|\left[1+\sigma^{2} \int \frac{1}{|z-x|^{2}} d \mu_{\sigma} \boxplus \nu(x)\right] \geq|\Im(z)|>0,(6.3) \\
\left|\Im\left[z-\gamma_{k}(1-c+c z g(z))\right]\right|=|\Im(z)|\left[1+c \gamma_{k} \int \frac{x}{|z-x|^{2}} d\left(\mu_{\mathrm{MP}, c} \otimes \nu\right)(x)\right] \geq|\Im(z)|>0 .
\end{gathered}
$$

According to (7.3) and Proposition 7.1 there exists a polynomial $P$ with nonnegative coefficients and a sequence $a_{N}$ of nonnegative real numbers converging to zero when $N$ goes to infinity such that, for any $k$ in $\{1, \ldots, N\}$, for any $z \in \mathbb{C} \backslash \mathbb{R}$

$$
\mathbb{E}\left(\tilde{G}_{k k}(z)\right)=\tilde{\Phi}_{N, k}(z)+R_{k, N}(z),
$$

with

$$
\left|R_{k, N}(z)\right| \leq(1+|z|)^{2} P\left(|\Im z|^{-1}\right) a_{N},
$$

where

$$
\tilde{\Phi}_{N, k}(z)= \begin{cases}\frac{1}{z-\sigma^{2} g_{N}^{W}(z)-\gamma_{k}}, & \text { if } M_{N}=M_{N}^{W}, \\ \frac{z-\gamma_{k}\left(1-\frac{N}{p}+\frac{N}{p} z g_{N}^{S}(z)\right)}{2} & \text { if } M_{N}=M_{N}^{S} .\end{cases}
$$

In order to deduce Proposition 6.2, we will need the following description of the convergence of $g_{N}(z)$ towards $g(z)$.

Proposition 6.3. There exists a polynomial $R$ with nonnegative coefficients, a sequence $a_{N}$ of positive numbers converging towards zero and some nonnegative integer number $\alpha$ such that, for all $z \in \mathbb{C} \backslash \mathbb{R}$,

$$
\left|g_{N}(z)-g(z)\right| \leq(1+|z|)^{\alpha} R\left(|\Im z|^{-1}\right) a_{N} .
$$

Proof: 1) The deformed Wigner model case.

Denote by $\tilde{g}_{N}$ the Stieltjes transform of $\mu_{\sigma} \boxplus \mu_{A_{N}}$. Since we have already proved in 24 that there exists a polynomial $S$ with nonnegative coefficients such that for all $z \in \mathbb{C} \backslash \mathbb{R}$,

$$
\left|\tilde{g}_{N}(z)-g_{N}(z)\right| \leq \frac{S\left(|\Im z|^{-1}\right)}{N}
$$


the result will readily follow if we prove that there exists a polynomial $T$ with nonnegative coefficients and a sequence $b_{N}$ of positive numbers converging towards zero such that, for all $z \in \mathbb{C}^{+}$,

$$
\left|\tilde{g}_{N}(z)-g(z)\right| \leq T\left(|\Im z|^{-1}\right) b_{N} .
$$

The proof of (6.8) follows the lines of Section 4 in [24. For a fixed $z \in \mathbb{C}^{+}$, according to Proposition 3.2, we have the subordination equations:

$$
\begin{gathered}
\tilde{g}_{N}(z)=g_{\mu_{A_{N}}}\left(F_{\sigma, \mu_{A_{N}}}^{(a)}(z)\right)=g_{\mu_{A_{N}}}\left(z-\sigma^{2} \tilde{g}_{N}(z)\right), \\
g(z)=g_{\nu}\left(F_{\sigma, \nu}^{(a)}(z)\right)=g_{\nu}\left(z-\sigma^{2} g(z)\right) .
\end{gathered}
$$

Moreover, using Lemma 7.7 and $\Im\left(z-\sigma^{2} g(z)\right) \geq \Im z$, we deduce from (6.10) that

$$
g(z)=g_{\mu_{A_{N}}}\left(z-\sigma^{2} g(z)\right)+\Delta_{N}(z)
$$

with

$$
\left|\Delta_{N}(z)\right| \leq v_{N}(1) P_{1}\left(|\Im z|^{-1}\right),
$$

where $P_{1}$ is a polynomial with nonnegative coefficients and $v_{N}(1)$ is a sequence of positive numbers converging towards zero.

Since $z-\sigma^{2} g(z) \in \mathbb{C}^{+}, z^{\prime} \in \mathbb{C}$ is well-defined by the formula :

$$
z^{\prime}:=H_{\sigma, \mu_{A_{N}}}\left(z-\sigma^{2} g(z)\right),
$$

where $H_{\sigma, \mu_{A_{N}}}$ is defined by (3.3) replacing $\nu$ by $\mu_{A_{N}}$. One has

$$
\begin{aligned}
\left|z^{\prime}-z\right| & =\left|-\sigma^{2}\left(g(z)-g_{\mu_{A_{N}}}\left(z-\sigma^{2} g(z)\right)\right)\right| \\
& \leq \sigma^{2} v_{N}(1) P_{1}\left(|\Im z|^{-1}\right) .
\end{aligned}
$$

- If

$$
\frac{|\Im z|}{2} \leq \sigma^{2} v_{N}(1) P_{1}\left(|\Im z|^{-1}\right)
$$

or equivalently

$$
\begin{aligned}
& 1 \leq 2 \sigma^{2}|\Im z|^{-1} P_{1}\left(|\Im z|^{-1}\right) v_{N}(1) \\
&\left|g(z)-\tilde{g}_{N}(z)\right| \leq \frac{2}{|\Im z|} \\
& \leq 4 \sigma^{2}|\Im z|^{-2} P_{1}\left(|\Im z|^{-1}\right) v_{N}(1) .
\end{aligned}
$$

- If

$$
\frac{|\Im z|}{2}>\sigma^{2} v_{N}(1) P_{1}\left(|\Im z|^{-1}\right)
$$

one has :

$$
\left|\Im z^{\prime}-\Im z\right| \leq\left|z^{\prime}-z\right| \leq \frac{|\Im z|}{2}
$$


which implies $\Im z^{\prime} \geq \frac{\Im z}{2}$ and therefore $z^{\prime} \in \mathbb{C}^{+}$. Hence, according to (3.4), it follows that $z-\sigma^{2} g(z) \in \Omega_{\sigma, \mu_{A_{N}}}$ (where $\Omega_{\sigma, \mu_{A_{N}}}$ is defined by (3.2) replacing $\nu$ by $\left.\mu_{A_{N}}\right)$ so that $F_{\sigma, \mu_{A_{N}}}^{(a)}\left(z^{\prime}\right)=z-\sigma^{2} g(z)$.

Thus, the approximative equation (6.11) may be rewritten

$$
g(z)=g_{\mu_{A_{N}}}\left(F_{\sigma, \mu_{A_{N}}}^{(a)}\left(z^{\prime}\right)\right)+\Delta_{N}(z)
$$

and then, using the subordination equation (6.9)

$$
g(z)=\tilde{g}_{N}\left(z^{\prime}\right)+\Delta_{N}(z) .
$$

Moreover,

$$
\begin{aligned}
\left|\tilde{g}_{N}\left(z^{\prime}\right)-\tilde{g}_{N}(z)\right| & =\left|\left(z-z^{\prime}\right) \int_{\mathbb{R}} \frac{d\left(\mu_{\sigma} \boxplus \mu_{A_{N}}\right)(x)}{\left(z^{\prime}-x\right)(z-x)}\right| \\
& \leq 2 \sigma^{2} v_{N}(1)|\Im z|^{-2} P_{1}\left(|\Im z|^{-1}\right) .
\end{aligned}
$$

Hence

$$
\begin{aligned}
\left|g(z)-\tilde{g}_{N}(z)\right| & \leq\left|g(z)-\tilde{g}_{N}\left(z^{\prime}\right)\right|+\left|\tilde{g}_{N}\left(z^{\prime}\right)-\tilde{g}_{N}(z)\right| \\
& \leq\left(2 \sigma^{2}|\Im z|^{-2}+1\right) v_{N}(1) P_{1}\left(|\Im z|^{-1}\right)
\end{aligned}
$$

Finally we get that for all $z \in \mathbb{C}^{+}$,

$$
\left|g(z)-\tilde{g}_{N}(z)\right| \leq\left(4 \sigma^{2}|\Im z|^{-2}+1\right) v_{N}(1) P_{1}\left(|\Im z|^{-1}\right)
$$

so that (6.6) is satisfied in the deformed Wigner model setting with $a_{N}=v_{N}(1)$ and $R(x)=\left(4 \sigma^{2} x^{2}+1\right) P_{1}(x), \alpha=0$.

2) The sample covariance matrix setting

Let $z$ be in $\mathbb{C}^{+}$. Note that it is obviously equivalent to prove such an estimation for $\left|\underline{g}_{N}(z)-g_{\tau_{c, \nu}}(z)\right|$ where

$$
\underline{g}_{N}(z)=\frac{1-\frac{N}{p}}{z}+\frac{N}{p} g_{N}(z)
$$

is the expected value of the Stieltjes transform of the spectral measure of $\underline{M}_{N}^{S}=$ $\frac{1}{p} B_{N}^{*} A_{N} B_{N}$. In the following any $P_{i}$ will denote a polynomial with nonnegative coefficients and $a_{N}(i)$ will denote a sequence of nonnegative numbers converging towards zero when $N$ goes to infinity. Letting the sum running over $k$ in (7.5) and dividing by $N$ we have

$$
g_{N}(z)=\int \frac{d \mu_{A_{N}}(t)}{z\left(1-\underline{g}_{N}(z)\right)}+\Delta_{N}(z)
$$

where

$$
\left|\Delta_{N}(z)\right| \leq(1+|z|)^{2} P_{1}\left(|\Im z|^{-1}\right) a_{N}(1)
$$


It readily follows that

$$
\underline{g}_{N}(z)=\frac{\underline{g}_{N}(z)}{z} \mathcal{Z}_{\frac{N}{p}, \mu_{A_{N}}}\left(\underline{g}_{N}(z)\right)+\frac{N}{p} \Delta_{N}(z)
$$

where $\mathcal{Z}_{\frac{N}{p}, \mu_{A_{N}}}$ is defined by (3.12) replacing $\nu$ by $\mu_{A_{N}}$ and $c$ by $\frac{N}{p}$. Then using Lemma 7.8 we deduce that

$$
\mathcal{Z}_{\frac{N}{p}, \mu_{A_{N}}}\left(\underline{g}_{N}(z)\right)=z+R_{N}(z)
$$

where for all large $N$

$$
\left|R_{N}(z)\right| \leq(1+|z|)^{5} P_{2}\left(|\Im z|^{-1}\right) a_{N}(1)
$$

On the other hand, using (7.22) and Lemma 7.8 we have

$$
\mathcal{Z}_{\frac{N}{p}, \mu_{A_{N}}}\left(\underline{g}_{N}(z)\right)=\mathcal{Z}\left(\underline{g}_{N}(z)\right)+Q_{N}(z)
$$

where

$$
\left|Q_{N}(z)\right| \leq(1+|z|)^{q} P_{3}\left(|\Im z|^{-1}\right) a_{N}(2)
$$

for some nonnegative integer number $q$. We readily deduce from (6.13) and (6.14) that

$$
\mathcal{Z}\left(\underline{g}_{N}(z)\right)=z+T_{N}(z)
$$

where

$$
\left|T_{N}(z)\right| \leq(1+|z|)^{\alpha} P_{4}\left(|\Im z|^{-1}\right) a_{N}(3)
$$

for some nonnegative integer number $\alpha$.

Set

$$
z^{\prime}=\mathcal{Z}\left(\underline{g}_{N}(z)\right)
$$

- If

$$
\frac{|\Im z|}{2} \leq(1+|z|)^{\alpha} P_{4}\left(|\Im z|^{-1}\right) a_{N}(3),
$$

or equivalently

$$
1 \leq 2|\Im z|^{-1}(1+|z|)^{\alpha} P_{4}\left(|\Im z|^{-1}\right) a_{N}(3),
$$

then

$$
\begin{aligned}
\left|g_{\tau_{c, \nu}}(z)-\underline{g}_{N}(z)\right| & \leq \frac{2}{|\Im z|} \\
& \leq 4|\Im z|^{-2}(1+|z|)^{\alpha} P_{4}\left(|\Im z|^{-1}\right) a_{N}(3)
\end{aligned}
$$

- If

$$
\frac{|\Im z|}{2}>(1+|z|)^{\alpha} P_{4}\left(|\Im z|^{-1}\right) a_{N}(3),
$$

one has :

$$
\left|\Im z^{\prime}-\Im z\right| \leq\left|z^{\prime}-z\right|<\frac{|\Im z|}{2}
$$


which implies $\Im z^{\prime} \geq \frac{\Im z}{2}$ and therefore $z^{\prime} \in \mathbb{C}^{+}$. Note that $\underline{g}_{N}(z)$ satisfied the equation

$$
Z=\frac{1}{z^{\prime}-c \int \frac{t}{1-t Z} d \nu(t)}
$$

and since $g_{\tau_{c, \nu}}\left(z^{\prime}\right)$ is the unique solution in $\mathbb{C}^{-}$of the latter equation, we can deduce that $g_{\tau_{c, \nu}}\left(z^{\prime}\right)=\underline{g}_{N}(z)$.

Hence

$$
\begin{aligned}
\left|\underline{g}_{N}(z)-g_{\tau_{c, \nu}}(z)\right| & =\left|g_{\tau_{c, \nu}}\left(z^{\prime}\right)-g_{\tau_{c, \nu}}(z)\right| \\
& \leq|\Im z|^{-1}\left|\Im z^{\prime}\right|^{-1}\left|z-z^{\prime}\right| \\
& \leq 2|\Im z|^{-2}(1+|z|)^{\alpha} P_{4}\left(|\Im z|^{-1}\right) a_{N}(3) .
\end{aligned}
$$

Finally we get that for all $z \in \mathbb{C}^{+}$,

$$
\left|\underline{g}_{N}(z)-g_{\tau_{c, \nu}}(z)\right| \leq 4|\Im z|^{-2}(1+|z|)^{\alpha} P_{4}\left(|\Im z|^{-1}\right) a_{N}(3)
$$

so that (6.6) is satisfied in the sample covariance matrix setting .

Now, Proposition 6.2 readily follows from (6.5) and Proposition 6.3 using (7.20), (6.4) and (7.4) and (6.3).

Thus, for any $l=1, \ldots, J$, (6.1) and Proposition 6.2 yield that for all large $N$, for all $z \in \mathbb{C} \backslash \mathbb{R}$,

$$
\mathbb{E}\left(\operatorname{Tr}\left[G_{M_{N}}(z) f_{\eta, l}\left(A_{N}\right)\right]\right)=\phi_{l}(z)+\Delta_{N}(z)
$$

where $\phi_{l}$ is the following analytic function on $\mathbb{C} \backslash \mathbb{R}$ :

$$
\phi_{l}(z)= \begin{cases}\frac{k_{l}}{z-\sigma^{2} g(z)-\theta_{l}}=\frac{k_{l}}{F_{\sigma, \nu}^{(a)}(z)-\theta_{l}} & \text { if } M_{N}=M_{N}^{W}, \\ \frac{k_{l}}{z-\theta_{l}(1-c+c z g(z))}=\frac{k_{l}}{z\left(1-\theta_{l} F_{c, \nu}^{(m)}\left(\frac{1}{z}\right)\right)} & \text { if } M_{N}=M_{N}^{S},\end{cases}
$$

and

$$
\left|\Delta_{N}(z)\right| \leq a_{N}(1+|z|)^{\alpha} P\left(\frac{1}{|\Im z|}\right)
$$

for some polynomial $P$ with nonnegative coefficients, some sequence $a_{N}$ of positive real numbers converging to zero when $N$ goes to infinity and some nonnegative integer number $\alpha$.

According to Lemma 8.5, we have

$$
\limsup _{y \rightarrow 0^{+}}\left(a_{N}\right)^{-1}\left|\int h_{\delta, j}(t) \Delta_{N}(t+i y) d t\right|<+\infty
$$

so that

$$
\lim _{N \rightarrow+\infty} \limsup _{y \rightarrow 0^{+}}\left|\int h_{\delta, j}(t) \Delta_{N}(t+i y) d t\right|=0 .
$$


Lemma 6.4. Let $\theta_{j}$ be in $\Theta_{o}$.

(1) Set $\rho_{\theta_{j}}=H\left(\theta_{j}\right)$. If $\theta_{l} \in \Theta \backslash\left\{\theta_{j}\right\}$, the map $(x, y) \mapsto F_{\sigma, \nu}^{(a)}(x+i y)-\theta_{l}$ does not vanish on $\left[\rho_{\theta_{j}}-2 \delta ; \rho_{\theta_{j}}+2 \delta\right] \times \mathbb{R}$.

The only vanishing point in $\left[\rho_{\theta_{j}}-2 \delta ; \rho_{\theta_{j}}+2 \delta\right] \times \mathbb{R}$ of the map $(x, y) \mapsto$ $F_{\sigma, \nu}^{(a)}(x+i y)-\theta_{j}$ is $\left(\rho_{\theta_{j}}, 0\right)$.

(2) Set $\rho_{\theta_{j}}=\mathcal{Z}\left(\frac{1}{\theta_{j}}\right)$. If $\theta_{l} \in \Theta \backslash\left\{\theta_{j}\right\}$, the map $(x, y) \mapsto(x+i y)\left(1-\theta_{l} g_{\tau_{c, \nu}}(x+\right.$ $i y)$ ) does not vanish on $\left[\rho_{\theta_{j}}-2 \delta ; \rho_{\theta_{j}}+2 \delta\right] \times \mathbb{R}$.

The only vanishing point in $\left[\rho_{\theta_{j}}-2 \delta ; \rho_{\theta_{j}}+2 \delta\right] \times \mathbb{R}$ of the map $(x, y) \mapsto$ $(x+i y)\left(1-\theta_{j} g_{\tau_{c, \nu}}(x+i y)\right)$ is $\left(\rho_{\theta_{j}}, 0\right)$.

Proof: Note that if $y \neq 0$, for any $x$, the imaginary part of $F_{\sigma, \nu}^{(a)}(x+i y)-\theta_{l}$ and $1-\theta_{l} g_{\tau_{c, \nu}}(x+i y)$ is nonnull so that we will focus on the case $y=0$. Proof of (1):

- Assume $\theta_{l} \notin \Theta_{o}$. First, if $H^{\prime}\left(\theta_{l}\right)<0$, according to (3.5), $\theta_{l}$ does not belong to $F_{\sigma, \nu}^{(a)}(\mathbb{R})$ so that the conclusion of Lemma 6.4 (1) is true.

Now assume that $H^{\prime}\left(\theta_{l}\right)=0$. According to (3.5), $\theta_{l} \in \partial \Omega_{\sigma, \nu}=F_{\sigma, \nu}^{(a)}(\mathbb{R})$, and, by Proposition 3.2 $F_{\sigma, \nu}^{(a)}(x)-\theta_{l}=0$ implies $x=H\left(\theta_{l}\right)$. For any $u \in \mathcal{O}^{(a)}$, we have $H\left(\theta_{l}\right) \neq H(u)$. Indeed, for any $u \in \mathcal{O}^{(a)}$, there exists $u_{1}, u_{2}$ such that $\left[u_{1} ; u_{2}\right] \subset \mathcal{O}^{(a)}$ and $u_{1}<u<u_{2}$. Since $H$ is globally nondecreasing on $\left\{v \in^{c} \operatorname{supp}(\nu), H^{\prime}(v) \geq 0\right\}$ (see Remark 3.3), we have if $\theta_{l}<u, H\left(\theta_{l}\right) \leq H\left(u_{1}\right)<H(u)$ and if $\theta_{l}>u, H(u)<H\left(u_{2}\right) \leq H\left(\theta_{l}\right)$. It follows, according to (4.1), that $H\left(\theta_{l}\right)$ belongs to $\operatorname{supp}(\mu \boxplus \sigma)$ and therefore cannot belong to $\left[\rho_{\theta_{j}}-2 \delta ; \rho_{\theta_{j}}+2 \delta\right]$ so that the conclusion of Lemma 6.4 (1) is true.

- Let us consider now, $\theta_{l} \in \Theta_{o}$. By Remark 3.2 and Proposition 3.2 . $F_{\sigma, \nu}^{(a)}(x)-\theta_{l}=0$ implies $x=H\left(\theta_{l}\right)=\rho_{\theta_{l}}$. If $l \neq j, \rho_{\theta_{l}}$ does not belong to $\left[\rho_{\theta_{j}}-2 \delta ; \rho_{\theta_{j}}+2 \delta\right]$ and the proof of Lemma [6.4(1) is complete.

Proof of (2):

First, note that $0 \notin\left[\rho_{\theta_{j}}-2 \delta ; \rho_{\theta_{j}}+2 \delta\right]$.

- Assume $\theta_{l} \notin \Theta_{o}$. First, if $\mathcal{Z}^{\prime}\left(\frac{1}{\theta_{l}}\right)>0$, according to Remark 3.8, $\frac{1}{\theta_{l}}$ does not belong to $g_{\tau_{c, \nu}}(\mathbb{R} \backslash\{0\})$ so that the conclusion of Lemma 6.4 (2) is true.

Now assume that $\mathcal{Z}^{\prime}\left(\frac{1}{\theta_{l}}\right)=0$. By Remark 3.4. $1-\theta_{l} g_{\tau_{c, \nu}}(x)=0$ with $x$ nonnull implies $x=\mathcal{Z}\left(\frac{1}{\theta_{l}}\right)$. In particular, if $\mathcal{Z}\left(\frac{1}{\theta_{l}}\right)=0$, the conclusion of Lemma 6.4 (2) is true since $0 \notin\left[\rho_{\theta_{j}}-2 \delta ; \rho_{\theta_{j}}+2 \delta\right]$. Hence, in the following, we will deal with $\theta_{l}$ such that $\mathcal{Z}\left(\frac{1}{\theta_{l}}\right) \neq 0$. For any $u \in \mathcal{O}^{(m)}$, we have $\mathcal{Z}\left(\frac{1}{\theta_{l}}\right) \neq \mathcal{Z}\left(\frac{1}{u}\right)$. Indeed, for any $u \in \mathcal{O}^{(m)}$, there exists $u_{1}, u_{2}$ such that $\left[u_{1} ; u_{2}\right] \subset \mathcal{O}^{(m)}$ and $u_{1}<u<u_{2}$. Since $x \mapsto \mathcal{Z}\left(\frac{1}{x}\right)$ is globally nondecreasing on $\left\{v \neq 0, v \in^{c} \operatorname{supp}(\nu), \mathcal{Z}^{\prime}\left(\frac{1}{v}\right) \leq 0\right\}$ (see Remark 3.6), we 
have if $\theta_{l}<u, \mathcal{Z}\left(\frac{1}{\theta_{l}}\right) \leq \mathcal{Z}\left(\frac{1}{u_{1}}\right)<\mathcal{Z}\left(\frac{1}{u}\right)$, and if $\theta_{l}>u, \mathcal{Z}\left(\frac{1}{u}\right)<\mathcal{Z}\left(\frac{1}{u_{2}}\right) \leq$ $\mathcal{Z}\left(\frac{1}{\theta_{l}}\right)$. It follows, according to (4.2), that $\mathcal{Z}\left(\frac{1}{\theta_{l}}\right)$ belongs to $\operatorname{supp}\left(\tau_{c, \nu}\right)$ and therefore cannot belong to $\left[\rho_{\theta_{j}}-2 \delta ; \rho_{\theta_{j}}+2 \delta\right]$ so that the conclusion of Lemma 6.4 (2) is true.

- Now, let us consider $\theta_{l} \in \Theta_{o}$. By Remark 3.4 $1-\theta_{l} g_{\tau_{c, \nu}}(x)=0$ with $x$ nonnull implies $x=\mathcal{Z}\left(\frac{1}{\theta_{l}}\right)=\rho_{\theta_{l}}$. If $l \neq j, \rho_{\theta_{l}}$ does not belong to $\left[\rho_{\theta_{j}}-2 \delta ; \rho_{\theta_{j}}+2 \delta\right]$ and the proof of Lemma 6.4 (2) is complete.

Let $\theta_{j}$ be in $\Theta_{o}$. According to Lemma 6.4 $\phi_{l}$ is an analytic function on $] \rho_{\theta_{j}}-$ $2 \delta ; \rho_{\theta_{j}}+2 \delta\left[\times \mathbb{R}\right.$ for $l \neq j$ and $\phi_{j}$ is an analytic function on $] \rho_{\theta_{j}}-2 \delta ; \rho_{\theta_{j}}+2 \delta[\times \mathbb{R} \backslash$ $\left\{\left(\rho_{\theta_{j}}, 0\right)\right\}$. Moreover, for any $l, \overline{\phi_{l}(z)}=\phi_{l}(\bar{z})$. We have

$$
\begin{aligned}
\frac{1}{\pi} \int \Im \phi_{l}(t+i y) h_{\delta, j}(t) d t= & \frac{1}{2 i \pi} \int_{\rho_{\theta_{j}}-\delta}^{\rho_{\theta_{j}}-\frac{\delta}{2}} h_{\delta, j}(t)\left[\phi_{l}(t+i y)-\phi_{l}(t-i y)\right] d t \\
& +\frac{1}{2 i \pi} \int_{\rho_{\theta_{j}}+\frac{\delta}{2}}^{\rho_{\theta_{j}}+\delta} h_{\delta, j}(t)\left[\phi_{l}(t+i y)-\phi_{l}(t-i y)\right] d t \\
& +\frac{1}{2 i \pi} \int_{\rho_{\theta_{j}}-\frac{\delta}{2}}^{\rho_{\theta_{j}}+\frac{\delta}{2}}\left[\phi_{l}(t+i y)-\phi_{l}(t-i y)\right] d t \\
= & \Delta_{1}+\Delta_{2}+\Delta_{3} .
\end{aligned}
$$

We immediately get that $\lim _{y \rightarrow 0^{+}} \Delta_{1}=0$ and $\lim _{y \rightarrow 0^{+}} \Delta_{2}=0$. Now,

$$
\begin{aligned}
\Delta_{3}= & \frac{1}{2 i \pi} \int_{\rho_{\theta_{j}-\frac{\delta}{2}}}^{\rho_{\theta_{j}}+\frac{\delta}{2}}\left[\phi_{l}(t+i y)-\phi_{l}(t-i y)\right] d t \\
= & \frac{1}{2 i \pi} \int_{\gamma_{j, y, \delta}} \phi_{l}(z) d z \\
& -\frac{1}{2 \pi} \int_{-y}^{y} \phi_{l}\left(\rho_{\theta_{j}}-\frac{\delta}{2}+i u\right) d u \\
& +\frac{1}{2 \pi} \int_{-y}^{y} \phi_{l}\left(\rho_{\theta_{j}}+\frac{\delta}{2}+i u\right) d u \\
= & \Delta_{3,1}+\Delta_{3,2}+\Delta_{3,3}
\end{aligned}
$$

where $\gamma_{j, y, \delta}$ is the clockwise oriented rectangular with corners $\rho_{\theta_{j}}-\frac{\delta}{2}-i y$, $\rho_{\theta_{j}}-\frac{\delta}{2}+i y, \rho_{\theta_{j}}+\frac{\delta}{2}+i y$ and $\rho_{\theta_{j}}+\frac{\delta}{2}-i y$. We immediately get that $\lim _{y \rightarrow 0^{+}} \Delta_{3,2}=$ 0 and $\lim _{y \rightarrow 0^{+}} \Delta_{3,3}=0$. Moreover, for all $y$,

$$
\Delta_{3,1}=\frac{1}{2 i \pi} \int_{\gamma_{j, y, \delta}} \phi_{l}(z) d z=-\operatorname{Res}\left(\phi_{l}, \rho_{\theta_{j}}\right)
$$

with

$$
\operatorname{Res}\left(\phi_{l}, \rho_{\theta_{j}}\right)=0 \text { if } l \neq j,
$$


and

$$
\operatorname{Res}\left(\phi_{j}, \rho_{\theta_{j}}\right)= \begin{cases}\frac{k_{j}}{F_{\sigma, \nu}^{(a)}\left(\rho_{\theta_{j}}\right)} & \text { if } M_{N}=M_{N}^{W} \\ =\frac{k_{j} \theta_{\theta_{j}}}{\theta_{j} F_{c, \nu}^{(m) \prime^{\prime}}\left(\frac{1}{\rho_{\theta_{j}}}\right)} & \text { if } M_{N}=M_{N}^{S}\end{cases}
$$

Thus

$$
\lim _{y \rightarrow 0^{+}} \frac{1}{\pi} \int h_{\delta, j}(t) \Im \phi_{l}(t+i y) d t=0 \text { if } l \neq j,
$$

and

$$
\lim _{y \rightarrow 0^{+}} \frac{1}{\pi} \int h_{\delta, j}(t) \Im \phi_{j}(t+i y) d t=\left\{\begin{array}{lr}
-k_{j} H^{\prime}\left(\theta_{j}\right) & \text { if } M_{N}=M_{N}^{W} \\
k_{j} \frac{\mathcal{Z}^{\prime}\left(\frac{1}{\theta_{j}}\right)}{\theta_{j}\left(\frac{1}{\theta_{j}}\right)} & \text { if } M_{N}=M_{N}^{S} .
\end{array}\right.
$$

Finally from (6.15), (6.16) (6.18) and (6.17) we deduce that

$$
\lim _{N \rightarrow+\infty} \lim _{y \rightarrow 0^{+}} \frac{1}{\pi} \Im \int \mathbb{E}\left(\operatorname{Tr}\left[G_{M_{N}}(t+i y) f_{\eta, l}\left(A_{N}\right)\right]\right) h_{\delta, j}(t) d t=0 \text { if } l \neq j,
$$

and

$$
\begin{aligned}
\lim _{N \rightarrow+\infty} \lim _{y \rightarrow 0^{+}} & \frac{1}{\pi} \Im \int \mathbb{E}\left(\operatorname{Tr}\left[G_{M_{N}}(t+i y) f_{\eta, j}\left(A_{N}\right)\right]\right) h_{\delta, j}(t) d t \\
= & \left\{\begin{array}{lc}
-k_{j} H^{\prime}\left(\theta_{j}\right) & \text { if } M_{N}=M_{N}^{W} \\
k_{j} \frac{\mathcal{Z}^{\prime}\left(\frac{1}{\theta_{j}}\right)}{\theta_{j}\left(\frac{1}{\theta_{j}}\right)} & \text { if } M_{N}=M_{N}^{S} .
\end{array}\right.
\end{aligned}
$$

Then, Proposition 4.1 follows by Proposition 6.1 and Lemma 6.3 .

\section{Technical results specific to each model}

\section{Lemma 7.1.}

(1) For any $N \times N$ Hermitian matrix $X$, $\left\{(X(i, i))_{1 \leq i \leq N}(\sqrt{2} \Re X(i, j))_{1 \leq i<j \leq N}(\sqrt{2} \Im X(i, j))_{1 \leq i<j \leq N}\right\}$

$$
\mapsto \operatorname{Tr}\left[h_{\delta, j}\left(X+A_{N}\right) f_{\eta, l}\left(A_{N}\right)\right]
$$

is Lipschitz with constant bounded by $\sqrt{k_{l}}\left\|h_{\delta, j}\right\|_{L i p}$.

(2) For any $N \times p$ matrix $B$,

$$
\left\{(\Re B(i, j), \Im B(i, j))_{1 \leq i \leq N, 1 \leq j \leq p}\right\} \mapsto \operatorname{Tr}\left[h_{\delta, j}\left(A_{N}^{\frac{1}{2}} B B^{*} A_{N}^{\frac{1}{2}}\right) f_{\eta, l}\left(A_{N}\right)\right]
$$

is Lipschitz with constant bounded by $\sqrt{k_{l}} \sqrt{2 C}\left\|\tilde{h}_{\delta, j}\right\|_{\text {Lip }}$ where $\tilde{h}_{\delta, j}(x)=$ $h_{\delta, j}\left(x^{2}\right)$ and $C=\sup _{N}\left\|A_{N}\right\|$. 
Proof Given two $N \times N$ Hermitian matrices $X$ and $X^{\prime}$, we have (using Lemma 8.2) that

$$
\begin{gathered}
\left|\operatorname{Tr}\left[h_{\delta, j}\left(X+A_{N}\right) f_{\eta, l}\left(A_{N}\right)\right]-\operatorname{Tr}\left[h_{\delta, j}\left(X^{\prime}+A_{N}\right) f_{\eta, l}\left(A_{N}\right)\right]\right| \\
\leq\left\|f_{\eta, l}\left(A_{N}\right)\right\|_{2}\left\|X-X^{\prime}\right\|_{2}\left\|h_{\delta, j}\right\|_{L i p}
\end{gathered}
$$

and (1) follows since $\left\|f_{\eta, l}\left(A_{N}\right)\right\|_{2}=\sqrt{k_{l}}$.

To prove (2) we will make use of a useful observation already made in 34. Let us introduce the $(N+p) \times(N+p)$ matrices

$$
\begin{gathered}
\mathcal{M}_{N+p}(B)=\left(\begin{array}{cc}
0_{p \times p} & B^{*} A_{N}^{\frac{1}{2}} \\
A_{N}^{\frac{1}{2}} B & 0_{N \times N}
\end{array}\right), \\
\mathcal{N}_{N+p}=\left(\begin{array}{cc}
0_{p \times p} & 0_{p \times N} \\
0_{N \times p} & f_{\eta, l}\left(A_{N}\right)
\end{array}\right) .
\end{gathered}
$$

It is easy to see that

$$
\operatorname{Tr}\left[h_{\delta, j}\left(A_{N}^{\frac{1}{2}} B B^{*} A_{N}^{\frac{1}{2}}\right) f_{\eta, l}\left(A_{N}\right)\right]=\operatorname{Tr}\left[\tilde{h}_{\delta, j}\left(\mathcal{M}_{N+p}(B)\right) \mathcal{N}_{N+p}\right],
$$

where $\tilde{h}_{\delta, j}(x)=h_{\delta, j}\left(x^{2}\right)$. Note that since $h_{\delta, j}$ is a $\mathcal{C}^{\infty}$ compactly supported function, $\tilde{h}_{\delta, j}$ is obviously a Lipschitz function. Hence

$$
\begin{aligned}
\left|\operatorname{Tr}\left[h_{\delta, j}\left(A_{N}^{\frac{1}{2}} B B^{*} A_{N}^{\frac{1}{2}}\right) f_{\eta, l}\left(A_{N}\right)\right]-\operatorname{Tr}\left[h_{\delta, j}\left(A_{N}^{\frac{1}{2}} B^{\prime}\left(B^{\prime}\right)^{*} A_{N}^{\frac{1}{2}}\right) f_{\eta, l}\left(A_{N}\right)\right]\right| \\
\quad=\left|\operatorname{Tr}\left[\tilde{h}_{\delta, j}\left(\mathcal{M}_{N+p}(B)\right) \mathcal{N}_{N+p}\right]-\operatorname{Tr}\left[\tilde{h}_{\delta, j}\left(\mathcal{M}_{N+p}\left(B^{\prime}\right)\right) \mathcal{N}_{N+p}\right]\right| \\
\leq\left\|\mathcal{N}_{N+p}\right\|_{2}\left\|\tilde{h}_{\delta, j}\left(\mathcal{M}_{N+p}(B)\right)-\tilde{h}_{\delta, j}\left(\mathcal{M}_{N+p}\left(B^{\prime}\right)\right)\right\|_{2} \\
\quad \leq \sqrt{k_{l}}\left\|\tilde{h}_{\delta, j}\right\|_{L i p}\left\|\mathcal{M}_{N+p}(B)-\mathcal{M}_{N+p}\left(B^{\prime}\right)\right\|_{2} .
\end{aligned}
$$

where we used Lemma 8.2 in the last line. Now,

$$
\begin{aligned}
\left\|\mathcal{M}_{N+p}(B)-\mathcal{M}_{N+p}\left(B^{\prime}\right)\right\|_{2}^{2} & =2 \operatorname{Tr}\left[\left(B-B^{\prime}\right)^{*} A_{N}\left(B-B^{\prime}\right)\right] \\
& \leq 2\left\|A_{N}\right\|\left\|B-B^{\prime}\right\|_{2}^{2} \\
& \leq 2 C\left\|B-B^{\prime}\right\|_{2}^{2} .
\end{aligned}
$$

(2) readily follows from (7.1) and (7.2).

We have proved in Lemma 3.3 24] that $\forall z \in \mathbb{C} \backslash \mathbb{R}$,

$$
\mathbb{E}\left(\tilde{G}_{k k}^{W}(z)\right)=\frac{1}{\left(z-\sigma^{2} g_{N}^{W}(z)-\gamma_{k}\right)}+R_{k, N}(z),
$$


with

$$
\left|R_{k, N}(z)\right| \leq \frac{P\left(|\Im z|^{-1}\right)}{N}
$$

for some polynomial $P$ with nonnegative coefficients. Note that

$$
\left|\Im\left(z-\sigma^{2} g_{N}^{W}(z)-\gamma_{k}\right)\right| \geq|\Im z| .
$$

We are going to establish the following similar result for the sample covariance matrix setting using many ideas from [7].

Proposition 7.1. There exists a polynomial $P$ with nonnegative coefficients and a sequence $a_{N}$ of nonnegative real numbers converging to zero when $N$ goes to infinity such that, for any $k$ in $\{1, \ldots, N\}$, any $z$ in $\mathbb{C} \backslash \mathbb{R}$,

$$
\mathbb{E}\left(\tilde{G}_{k k}^{S}(z)\right)=\frac{1}{z-\gamma_{k}\left(1-\frac{N}{p}+\frac{N}{p} z g_{N}^{S}(z)\right)}+R_{k, N}(z),
$$

with

$$
\left|R_{k, N}(z)\right| \leq(1+|z|)^{2} P\left(|\Im z|^{-1}\right) a_{N} .
$$

Proof Let $J$ be a $N \times N$ matrix and $u$ be a vector in $\mathbb{C}^{N}$ such that $J$ and $J+u u^{*}$ are invertible then

$$
\begin{aligned}
u^{*} J^{-1} u u^{*}\left(J+u u^{*}\right)^{-1} & =u^{*} J^{-1}\left(u u^{*}+J\right)\left(J+u u^{*}\right)^{-1}-u^{*}\left(J+u u^{*}\right)^{-1} \\
& =u^{*} J^{-1}-u^{*}\left(J+u u^{*}\right)^{-1}
\end{aligned}
$$

so that

$$
u^{*}\left(J+u u^{*}\right)^{-1}=\frac{u^{*} J^{-1}}{1+u^{*} J^{-1} u} .
$$

Hence if $u_{1}, \ldots, u_{p}$ are $p$ vectors and $X=\sum_{i=1}^{p} u_{i} u_{i}^{*}$, denoting by $G_{X}(z)$ the resolvent $(z I-X)^{-1}$, (7.6) yields that for any $i \in\{1, \ldots, p\}$, for any $z \in \mathbb{C} \backslash \mathbb{R}$,

$$
u_{i} G_{X}(z)=\frac{u_{i}^{*}\left(z I-\sum_{l \neq i} u_{l} u_{l}^{*}\right)^{-1}}{1-u_{i}^{*}\left(z I-\sum_{l \neq i} u_{l} u_{l}^{*}\right)^{-1} u_{i}} .
$$

Multiplying (17.7) by $u_{i}$ and summing in $i$ yields

$$
X G_{X}(z)=\sum_{i=1}^{p} \frac{u_{i} u_{i}^{*}\left(z I-\sum_{l \neq i} u_{l} u_{l}^{*}\right)^{-1}}{1-u_{i}^{*}\left(z I-\sum_{l \neq i} u_{l} u_{l}^{*}\right)^{-1} u_{i}} .
$$

From (7.8) and the resolvent identity

$$
-I+z G_{X}(z)=X G_{X}(z),
$$


we deduce that

$$
\left(G_{X}(z)\right)_{k k}=\frac{1}{z}+\sum_{i=1}^{p} \frac{\left[u_{i} u_{i}^{*}\left(z I-\sum_{l \neq i} u_{l} u_{l}^{*}\right)^{-1}\right]_{k k}}{z\left[1-u_{i}^{*}\left(z I-\sum_{l \neq i} u_{l} u_{l}^{*}\right)^{-1} u_{i}\right]} .
$$

Noticing that

$$
\tilde{G}^{S}(z)=G_{\frac{1}{p} D^{\frac{1}{2}} U X_{N}^{S} U^{*} D^{\frac{1}{2}}}
$$

and that

$$
\frac{1}{p} D^{\frac{1}{2}} U X_{N}^{S} U^{*} D^{\frac{1}{2}}=\sum_{i=1}^{p} u_{i} u_{i}^{*}
$$

where $u_{i}=\frac{1}{\sqrt{p}} D^{\frac{1}{2}} U x_{i}$ and $x_{i}$ is the $i$ th column of $B_{N}$, we deduce from (7.9) that

$$
\tilde{G}_{k k}^{S}(z)=\frac{1}{z}+\frac{1}{p} \sum_{i=1}^{p} \frac{\left[D^{\frac{1}{2}} U x_{i} x_{i}^{*} U^{*} D^{\frac{1}{2}}\left(z I-\frac{1}{p} \sum_{l \neq i} D^{\frac{1}{2}} U x_{l} x_{l}^{*} U^{*} D^{\frac{1}{2}}\right)^{-1}\right]_{k k}}{z\left\{\frac{1}{p} x_{i}^{*} U^{*} D^{\frac{1}{2}}\left(z I-\frac{1}{p} \sum_{l \neq i} D^{\frac{1}{2}} U x_{l} x_{l}^{*} U^{*} D^{\frac{1}{2}}\right)^{-1} D^{\frac{1}{2}} U x_{i}\right\}} .
$$

Set for $i=1, \ldots, p$,

$$
y_{i}=\frac{1}{\sqrt{p}} A_{N}^{\frac{1}{2}} x_{i}
$$

and

$$
M_{N}^{(i)}=\frac{1}{p} \sum_{l \neq i} A_{N}^{\frac{1}{2}} x_{l} x_{l}^{*} A_{N}^{\frac{1}{2}}=\sum_{l \neq i} y_{l} y_{l}^{*} .
$$

Note that the $y_{i}$ 's are i.i.d and that $y_{i}$ is independent of $M_{N}^{(i)}$. Note also that

$$
M_{N}^{S}=M_{N}^{(i)}+y_{i} y_{i}^{*}
$$

(7.11) can be rewritten as follows

$$
\tilde{G}_{k k}^{S}(z)=\frac{1}{z}+\frac{1}{p} \sum_{i=1}^{p} \frac{\left[D^{\frac{1}{2}} U x_{i} x_{i}^{*} A_{N}^{\frac{1}{2}}\left(z I-M_{N}^{(i)}\right)^{-1} U^{*}\right]_{k k}}{z\left\{1-y_{i}^{*}\left(z I-M_{N}^{(i)}\right)^{-1} y_{i}\right\}}
$$

Now applying (7.9) with $u_{i}=y_{i}$ and $X=M_{N}^{S}$, summing on $k$ and dividing by $N$ we have

$$
\begin{aligned}
\operatorname{tr}_{N}\left(G_{M_{N}^{S}}(z)\right) & =\frac{1}{z}+\frac{1}{N} \sum_{i=1}^{p} \frac{y_{i}^{*}\left(z I-M_{N}^{(i)}\right)^{-1} y_{i}}{z\left\{1-y_{i}^{*}\left(z I-M_{N}^{(i)}\right)^{-1} y_{i}\right\}} \\
& =\frac{1}{z}-\frac{p}{N} \frac{1}{z}+\frac{1}{N} \sum_{i=1}^{p} \frac{1}{z\left\{1-y_{i}^{*}\left(z I-M_{N}^{(i)}\right)^{-1} y_{i}\right\}}
\end{aligned}
$$


Let us define the $p \times p$ matrix

$$
\underline{M}_{N}^{S}=\frac{1}{p} B_{N}^{*} A_{N} B_{N} .
$$

Since

$$
\operatorname{tr}_{p}\left(G_{\underline{M}_{N}^{S}}(z)\right)=\frac{1-\frac{N}{p}}{z}+\frac{N}{p} \operatorname{tr}_{N}\left(G_{M_{N}^{S}}(z)\right),
$$

we deduce from (7.13) that

$$
\operatorname{tr}_{N}\left(G_{\underline{M}_{N}^{S}}(z)\right)=\frac{1}{p} \sum_{i=1}^{p} \frac{1}{z\left\{1-y_{i}^{*}\left(z I-M_{N}^{(i)}\right)^{-1} y_{i}\right\}} .
$$

Following the ideas of Section 6.4.1 of [7], we are going to establish the following preliminary lemma.

Lemma 7.2. There exists a constant $K>0$ and a sequence of nonnegative numbers $a_{N}$ converging to zero when $N$ goes to infinity such that for each $i=$ $1, \ldots, p, \forall z \in \mathbb{C} \backslash \mathbb{R}$

$$
\left\|\operatorname{tr}_{p}\left(G_{\underline{M}_{N}^{S}}(z)\right)-\frac{1}{z\left\{1-y_{i}^{*}\left(z I-M_{N}^{(i)}\right)^{-1} y_{i}\right\}}\right\|_{L^{2}} \leq K \frac{|z|}{|\Im z|^{3}} a_{N} .
$$

We have from (7.15)

$$
\begin{aligned}
\operatorname{tr}_{p}\left(G_{\underline{M}_{N}^{S}}(z)\right)-\frac{1}{z\left\{1-y_{i}^{*}\left(z I-M_{N}^{(i)}\right)^{-1} y_{i}\right\}} \\
\quad=\frac{1}{p} \sum_{l \neq i} \frac{y_{l}^{*}\left(z I-M_{N}^{(l)}\right)^{-1} y_{l}-y_{i}^{*}\left(z I-M_{N}^{(i)}\right)^{-1} y_{i}}{z\left\{1-y_{l}^{*}\left(z I-M_{N}^{(l)}\right)^{-1} y_{l}\right\}\left\{1-y_{i}^{*}\left(z I-M_{N}^{(i)}\right)^{-1} y_{i}\right\}} .
\end{aligned}
$$

Lemma 7.3. For any $N \times N$ positive semidefinite matrix $H$, any vector $v$ in $\mathbb{C}^{N}$ and any $z$ in $\mathbb{C} \backslash \mathbb{R}$,

$$
\frac{1}{\left|z\left\{1-v^{*}(z I-H)^{-1} v\right\}\right|} \leq \frac{1}{|\Im z|} .
$$

\section{Proof:}

$$
\begin{aligned}
\Im\left\{z v^{*}(z I-H)^{-1} v\right\} & =\frac{1}{2 i}\left\{z v^{*}(z I-H)^{-1} v-\bar{z} v^{*}(\bar{z} I-H)^{-1} v\right\} \\
& =\frac{1}{2 i} v^{*}\left\{\left(I-\frac{1}{z} H\right)^{-1}-\left(I-\frac{1}{\bar{z}} H\right)^{-1}\right\} v \\
& =-\frac{\Im z}{|z|^{2}} v^{*}\left(I-\frac{1}{z} H\right)^{-1} H\left(I-\frac{1}{\bar{z}} H\right)^{-1} v .
\end{aligned}
$$


Hence

$$
\begin{aligned}
\left|\Im\left[z\left\{1-v^{*}(z I-H)^{-1} v\right\}\right]\right| & =|\Im z|\left\{1+\frac{\mathbf{1}}{|z|^{2}} v^{*}\left(I-\frac{1}{z} H\right)^{-1} H\left(I-\frac{1}{\bar{z}} H\right)^{-1} v\right\} \\
& \geq|\Im z|
\end{aligned}
$$

and Lemma 7.3 follows. $\square$

According to Lemma 7.3. for any $l$ and $i$ in $\{1, \ldots, p\}$,

$$
\frac{1}{\left|z\left\{1-y_{l}^{*}\left(z I-M_{N}^{(l)}\right)^{-1} y_{l}\right\}\left\{1-y_{i}^{*}\left(z I-M_{N}^{(i)}\right)^{-1} y_{i}\right\}\right|} \leq \frac{|z|}{|\Im z|^{2}} .
$$

Hence

$$
\begin{aligned}
& \left\|\operatorname{tr}_{p}\left(G_{\underline{M}_{N}^{S}}(z)\right)-\frac{1}{z\left\{1-y_{i}^{*}\left(z I-M_{N}^{(i)}\right)^{-1} y_{i}\right\}}\right\|_{L^{2}} \\
& \quad \leq \frac{|z|}{|\Im z|^{2}} \frac{1}{p} \sum_{l \neq i}\left\|y_{l}^{*}\left(z I-M_{N}^{(l)}\right)^{-1} y_{l}-y_{i}^{*}\left(z I-M_{N}^{(i)}\right)^{-1} y_{i}\right\|_{L^{2}} .
\end{aligned}
$$

We have

$$
\begin{aligned}
y_{l}^{*}(z I- & \left.M_{N}^{(l)}\right)^{-1} y_{l}-y_{i}^{*}\left(z I-M_{N}^{(i)}\right)^{-1} y_{i} \\
= & y_{l}^{*}\left(z I-M_{N}^{(l)}\right)^{-1} y_{l}-\frac{1}{p} \operatorname{Tr}\left[\left(z I-M_{N}^{(l)}\right)^{-1} A_{N}\right] \\
& +\frac{1}{p} \operatorname{Tr}\left[\left(z I-M_{N}^{(l)}\right)^{-1} A_{N}\right]-\frac{1}{p} \operatorname{Tr}\left[\left(z I-M_{N}^{(i)}\right)^{-1} A_{N}\right] \\
& +\frac{1}{p} \operatorname{Tr}\left[\left(z I-M_{N}^{(i)}\right)^{-1} A_{N}\right]-y_{i}^{*}\left(z I-M_{N}^{(i)}\right)^{-1} y_{i} \\
= & \Delta_{l}+\Delta_{l, i}-\Delta_{i} .
\end{aligned}
$$

We have

$$
\begin{aligned}
\left|\Delta_{l, i}\right| \leq & \left|\frac{1}{p} \operatorname{Tr}\left[\left\{\left(z I-M_{N}^{(l)}\right)^{-1}-\left(z I-M_{N}^{S}\right)^{-1}\right\} A_{N}\right]\right| \\
& +\left|\frac{1}{p} \operatorname{Tr}\left[\left\{\left(z I-M_{N}^{S}\right)^{-1}-\left(z I-M_{N}^{(i)}\right)^{-1}\right\} A_{N}\right]\right| \\
\leq & \frac{2\left\|A_{N}\right\|}{|\Im z| p} \leq \frac{2 C}{|\Im z| p}
\end{aligned}
$$

where we used Lemma 6.9 [7]) in the last line.

Now we have for any $l=1, \ldots, p$,

$$
y_{l}^{*}\left(z I-M_{N}^{(l)}\right)^{-1} y_{l}=\frac{1}{p} x_{l}^{*} A_{N}^{\frac{1}{2}}\left(z I-M_{N}^{(l)}\right)^{-1} A_{N}^{\frac{1}{2}} x_{l}
$$


so that according to Proposition 8.1 in the Appendix, for any $l=1, \ldots, p$,

$$
\left\|\Delta_{l}\right\|_{L^{2}} \leq \sqrt{K} \sqrt{\frac{N}{p}} \frac{1}{\sqrt{p}} \frac{\left\|A_{N}\right\|}{|\Im z|} \leq \sqrt{\frac{N}{p}} \frac{1}{\sqrt{p}} \frac{C}{|\Im z|} .
$$

It follows that

$$
\left\|\operatorname{tr}_{p}\left(G_{\underline{M}_{N}^{S}}(z)\right)-\frac{1}{z\left\{1-y_{i}^{*}\left(z I-M_{N}^{(i)}\right)^{-1} y_{i}\right\}}\right\|_{L^{2}} \leq \frac{2 C|z|}{|\Im z|^{3}}\left(\frac{1}{p}+\sqrt{\frac{N}{p}} \frac{1}{\sqrt{p}}\right)
$$

and the proof of Lemma 7.2 is complete.

Lemma 7.4. There exists a constant $K>0$ such that, for any $k=1, \ldots, N$, for any $i=1, \ldots, p$ and any $z$ in $\mathbb{C} \backslash \mathbb{R}$,

$$
\left\|\left[D^{\frac{1}{2}} U x_{i} x_{i}^{*} A_{N}^{\frac{1}{2}}\left(z I-M_{N}^{(i)}\right)^{-1} U^{*}\right]_{k k}\right\|_{L^{2}} \leq \frac{K}{|\Im z|} .
$$

Proof: Note that

$$
\begin{aligned}
{\left[D^{\frac{1}{2}} U x_{i} x_{i}^{*} A_{N}^{\frac{1}{2}}\left(z I-M_{N}^{(i)}\right)^{-1} U^{*}\right]_{k k} } & =\operatorname{Tr} D^{\frac{1}{2}} U x_{i} x_{i}^{*} A_{N}^{\frac{1}{2}}\left(z I-M_{N}^{(i)}\right)^{-1} U^{*} E_{k k} \\
& =x_{i}^{*} A_{N}^{\frac{1}{2}}\left(z I-M_{N}^{(i)}\right)^{-1} U^{*} E_{k k} D^{\frac{1}{2}} U x_{i} .
\end{aligned}
$$

Thus, according to Proposition 8.1 in the Appendix,

$$
\begin{aligned}
& \left\|\left[D^{\frac{1}{2}} U x_{i} x_{i}^{*} A_{N}^{\frac{1}{2}}\left(z I-M_{N}^{(i)}\right)^{-1} U^{*}\right]_{k k}-\operatorname{Tr} A_{N}^{\frac{1}{2}}\left(z I-M_{N}^{(i)}\right)^{-1} U^{*} E_{k k} D^{\frac{1}{2}} U\right\|_{L^{2}} \\
& \leq K\left[\operatorname{Tr} A_{N}^{\frac{1}{2}}\left(z I-M_{N}^{(i)}\right)^{-1} U^{*} E_{k k} D E_{k k} U\left(\bar{z} I-M_{N}^{(i)}\right)^{-1} A_{N}^{\frac{1}{2}}\right]^{\frac{1}{2}} \leq \frac{C}{|\Im z|} .
\end{aligned}
$$

Since moreover

$$
\left|\operatorname{Tr} A_{N}^{\frac{1}{2}}\left(z I-M_{N}^{(i)}\right)^{-1} U^{*} E_{k k} D^{\frac{1}{2}} U\right| \leq \frac{C}{|\Im z|},
$$

we deduce that

$$
\left\|\left[D^{\frac{1}{2}} U x_{i} x_{i}^{*} A_{N}^{\frac{1}{2}}\left(z I-M_{N}^{(i)}\right)^{-1} U^{*}\right]_{k k}\right\|_{L^{2}} \leq \frac{2 C}{|\Im z|} .
$$

We will need this last lemma concerning the variance of $\operatorname{tr}_{p}\left(G_{\underline{M}_{N}^{S}}(z)\right)$.

Lemma 7.5. There exists some polynomial $P$ with nonnegative coefficients such that, $\forall z \in \mathbb{C} \backslash \mathbb{R}$,

$$
\left\|\operatorname{tr}_{p}\left(G_{\underline{M}_{N}^{S}}(z)\right)-\mathbb{E}\left(\operatorname{tr}_{p}\left(G_{\underline{M}_{N}^{S}}(z)\right)\right)\right\|_{L^{2}} \leq \frac{1}{p^{2}}(|z|+1)^{2} P\left(|\Im z|^{-1}\right) .
$$


Proof: Let us define $\Psi: \mathbb{R}^{2(p \times N)} \rightarrow \mathcal{M}_{N \times p}(\mathbb{C})$ by

$\Psi:\left\{x_{i j}, y_{i j}, i=1, \ldots, N, j=1, \ldots, p\right\} \rightarrow \sum_{i=1, \ldots, N} \sum_{j=1, \ldots, p}\left(x_{i j}+\sqrt{-1} y_{i j}\right) E_{i j}$.

Let $F$ be a smooth complex function on $M_{N \times p}(\mathbb{C})$ and define the complex function $f$ on $\mathbb{R}^{2(p \times N)}$ by setting $f=F \circ \Psi$. Then,

$$
\|\operatorname{grad} f(u)\|=\sup _{V \in M_{N \times p}(\mathbb{C}), T r V V^{*}=1}\left|\frac{d}{d t} F(\Psi(u)+t V)\right|_{t=0} \mid .
$$

We have $B_{N}=\Psi\left(\Re\left(\left(B_{N}\right)_{i j}\right), \Im\left(\left(B_{N}\right)_{i j}\right), 1 \leq i \leq N, 1 \leq j \leq p\right)$ where the distribution of $\left\{\sqrt{2} \Re\left(\left(B_{N}\right)_{i j}\right), \sqrt{2} \Im\left(\left(B_{N}\right)_{i j}\right), 1 \leq i \leq N, 1 \leq j \leq p\right\}$ satisfies a Poincaré inequality with constant $C_{P I}$.

Hence consider $F: B \rightarrow \operatorname{tr}_{N}\left(z I_{N}-A_{N}^{\frac{1}{2}} \frac{B B^{*}}{p} A_{N}^{\frac{1}{2}}\right)^{-1}$.

Let $V \in M_{N \times p}(\mathbb{C})$ such that $\operatorname{Tr} V V^{*}=1$.

$$
\begin{aligned}
\left.\frac{d}{d t} F(B+t V)\right|_{t=0}= & \frac{1}{N p} \operatorname{Tr}\left(G_{M_{N}^{S}}(z) A_{N}^{\frac{1}{2}} V B^{*} A_{N}^{\frac{1}{2}} G_{M_{N}^{S}}(z)\right) \\
& +\frac{1}{N p} \operatorname{Tr}\left(G_{M_{N}^{S}}(z) A_{N}^{\frac{1}{2}} B V^{*} A_{N}^{\frac{1}{2}} G_{M_{N}^{S}}(z)\right) .
\end{aligned}
$$

By Cauchy-Schwartz inequality, we have

$$
\begin{aligned}
& \left|\frac{1}{N p} \operatorname{Tr}\left(G_{M_{N}^{S}}(z) A_{N}^{\frac{1}{2}} V B_{N}^{*} A_{N}^{\frac{1}{2}} G_{M_{N}^{S}}(z)\right)\right| \\
& \quad \leq \frac{1}{N \sqrt{p}}\left[\operatorname{Tr} \frac{B_{N}^{*} A_{N}^{\frac{1}{2}}\left[G_{M_{N}^{S}}(z)\right]^{2} A_{N}\left[G_{M_{N}^{S}}(\bar{z})\right]^{2} A_{N}^{\frac{1}{2}} B_{N}}{p}\right]^{\frac{1}{2}}\left(\operatorname{Tr} V V^{*}\right)^{\frac{1}{2}} \\
& \quad=\frac{1}{\sqrt{N p}}\left[\operatorname{tr}_{N} M_{N}^{S}\left[G_{M_{N}^{S}}(z)\right]^{2} A_{N}\left[G_{M_{N}^{S}}(\bar{z})\right]^{2}\right]^{\frac{1}{2}} .
\end{aligned}
$$

Since by the resolvent identity

$$
M_{N}^{S} G_{M_{N}^{S}}(z)=-I_{N}+z G_{M_{N}^{S}},
$$

we have

$$
\begin{aligned}
& \operatorname{tr}_{N} M_{N}^{S}\left[G_{M_{N}^{S}}(z)\right]^{2} A_{N}\left[G_{M_{N}^{S}}(\bar{z})\right]^{2} \\
& \quad=-\operatorname{tr}_{N} G_{M_{N}^{S}}(z) A_{N}\left[G_{M_{N}^{S}}(\bar{z})\right]^{2}+z \operatorname{tr}_{N}\left[G_{M_{N}^{S}}(z)\right]^{2} A_{N}\left[G_{M_{N}^{S}}(\bar{z})\right]^{2},
\end{aligned}
$$

we can deduce that

$\operatorname{tr}_{N} M_{N}^{S}\left[G_{M_{N}^{S}}(z)\right]^{2} A_{N}\left[G_{M_{N}^{S}}(\bar{z})\right]^{2} \leq \frac{\left\|A_{N}\right\|}{|\Im z|^{3}}+\frac{|z|\left\|A_{N}\right\|}{|\Im z|^{4}} \leq C(|z|+1) P\left(|\Im z|^{-1}\right)$, 
where $P$ is a polynomial with non negative coefficients. Hence

$$
\left|\frac{1}{N p} \operatorname{Tr}\left(G_{M_{N}^{S}}(z) A_{N}^{\frac{1}{2}} V B_{N}^{*} A_{N}^{\frac{1}{2}} G_{M_{N}^{S}}(z)\right)\right| \leq \frac{1}{\sqrt{N p}} C(|z|+1) P\left(|\Im z|^{-1}\right) .
$$

Since a similar upper bound can be obtained in the same way for the second term on the right hand side of (7.16) we deduce that

$\mathbb{E}\left(\left(\sup _{V \in M_{N \times p}(\mathbb{C}), T r V V^{*}=1}\left|\frac{d}{d t} F\left(B_{N}+t V\right)\right|_{t=0} \mid\right)^{2}\right)^{\frac{1}{2}} \leq \frac{2 C}{\sqrt{N p}}(|z|+1) P\left(|\Im z|^{-1}\right)$.

Therefore, Poincaré inequality yields

$$
\left\|\operatorname{tr}_{N}\left(G_{M_{N}^{S}}(z)\right)-\mathbb{E}\left(\operatorname{tr}_{N}\left(G_{M_{N}^{S}}(z)\right)\right)\right\|_{L^{2}} \leq \frac{1}{N p}(|z|+1)^{2} Q\left(|\Im z|^{-1}\right)
$$

where $Q$ is a polynomial with non negative coefficients. Now, since

$\operatorname{tr}_{p}\left(G_{\underline{M}_{N}^{S}}(z)\right)-\mathbb{E}\left(\operatorname{tr}_{p}\left(G_{\underline{M}_{N}^{S}}(z)\right)\right)=\frac{N}{p}\left[\operatorname{tr}_{N}\left(G_{M_{N}^{S}}(z)\right)-\mathbb{E}\left(\operatorname{tr}_{N}\left(G_{M_{N}^{S}}(z)\right)\right)\right]$,

Lemma 7.5 follows.

Using (7.12), we have for any $z$ in $\mathbb{C} \backslash \mathbb{R}$,

$$
\begin{aligned}
\mathbb{E}\left(\tilde{G}_{k k}^{S}(z)\right)= & \frac{1}{z}+\frac{1}{p} \sum_{i=1}^{p} \mathbb{E}\left(\Phi_{i, k}\right) \mathbb{E}\left(\operatorname{tr}_{p} G_{\underline{M}_{N}^{S}}(z)\right) \\
& +\frac{1}{p} \sum_{i=1}^{p} \mathbb{E}\left[\Phi_{i, k}\left\{\operatorname{tr}_{p} G_{\underline{M}_{N}^{S}}(z)-\mathbb{E}\left(\operatorname{tr}_{p} G_{\underline{M}_{N}^{S}}(z)\right)\right\}\right] \\
& +\frac{1}{p} \sum_{i=1}^{p} \mathbb{E}\left[\Phi_{i, k}\left\{\frac{1}{z\left\{1-y_{i}^{*}\left(z I-M_{N}^{(i)}\right)^{-1} y_{i}\right\}}-\operatorname{tr}_{p} G_{\underline{M}_{N}^{S}}(z)\right\}\right]
\end{aligned}
$$

where

$$
\Phi_{i, k}=\left[D^{\frac{1}{2}} U x_{i} x_{i}^{*} A_{N}^{\frac{1}{2}}\left(z I-M_{N}^{(i)}\right)^{-1} U^{*}\right]_{k k} .
$$

By Cauchy-Schwartz inequality, using Lemmas 7.4 and 7.2, we easily have that there exists a constant $K$ and a sequence of nonnegative numbers $a_{N}$ converging towards zero when $N$ goes to infinity such that for any $k=1, \ldots, N$,

$$
\mid \frac{1}{p} \sum_{i=1}^{p} \mathbb{E}\left[\Phi_{i, k}\left\{\frac{1}{z\left\{1-y_{i}^{*}\left(z I-M_{N}^{(i)}\right)^{-1} y_{i}\right\}}-\operatorname{tr}_{p} G_{\underline{M}_{N}^{S}}(z)\right\} \mid \leq \frac{K|z|}{|\Im z|^{4}} a_{N} .\right.
$$


By Cauchy-Schwartz inequality, using Lemmas 7.4 and 7.5 we also have that there exists a polynomial $P$ with nonnegative coefficients such that

$$
\left|\frac{1}{p} \sum_{i=1}^{p} \mathbb{E}\left[\Phi_{i, k}\left\{\operatorname{tr}_{p} G_{\underline{M}_{N}^{S}}(z)-\mathbb{E}\left(\operatorname{tr}_{p} G_{\underline{M}_{N}^{S}}(z)\right)\right\}\right]\right| \leq \frac{1}{p^{2}}(|z|+1)^{2} P\left(|\Im z|^{-1}\right) .
$$

Thus

$$
\mathbb{E}\left(\tilde{G}_{k k}^{S}(z)\right)=\frac{1}{z}+\frac{1}{p} \sum_{i=1}^{p} \mathbb{E}\left(\Phi_{i, k}\right) \mathbb{E}\left(\operatorname{tr}_{p} G_{\underline{M}_{N}^{S}}(z)\right)+\Delta_{N}(k)
$$

where there exists a polynomial $Q$ with nonnegative coefficients and a sequence of nonnegative numbers $b_{N}$ converging towards zero when $N$ goes to infinity such that, for any $k=1, \ldots, N$,

$$
\left|\Delta_{N}(k)\right| \leq(|z|+1)^{2} Q\left(|\Im z|^{-1}\right) b_{N} .
$$

Now, one can easily see that

$$
\begin{aligned}
\mathbb{E}\left(\Phi_{i, k}\right) & =\gamma_{k} \mathbb{E}\left(\left[U\left(z I-M_{N}^{(i)}\right)^{-1} U^{*}\right]_{k k}\right) \\
& =\gamma_{k} \mathbb{E}\left(\left[\left(z I-\sum_{l \neq i} u_{l} u_{l}^{*}\right)^{-1}\right]_{k k}\right)
\end{aligned}
$$

where

$$
u_{i}=\frac{1}{\sqrt{p}} D^{\frac{1}{2}} U x_{i} .
$$

Lemma 7.6. There exists a polynomial $P$ with nonnegative coefficients such that for any $i=1, \ldots, p$, any $k=1, \ldots, N$, any $z \in \mathbb{C} \backslash \mathbb{R}$,

$$
\left|\mathbb{E}\left(\tilde{G}_{k k}^{S}(z)\right)-\mathbb{E}\left(\left[\left(z I-\sum_{l \neq i} u_{l} u_{l}^{*}\right)^{-1}\right]_{k k}\right)\right| \leq \frac{1}{p}(|z|+1) P\left(|\Im z|^{-1}\right) .
$$

Proof: Remember that according to (7.10),

$$
\mathbb{E}\left(\tilde{G}_{k k}^{S}(z)\right)=\mathbb{E}\left(\left[\left(z I-\sum_{l=1}^{p} u_{l} u_{l}^{*}\right)^{-1}\right]_{k k}\right) .
$$

By the formula (3.3.4) in [7, we have

$$
\left[\left(z I-\sum_{l=1}^{p} u_{l} u_{l}^{*}\right)^{-1}\right]_{k k}=\left[\left(z I-\sum_{l \neq i} u_{l} u_{l}^{*}\right)^{-1}\right]_{k k}+\frac{\psi_{i, k}}{1-u_{i}^{*}\left(z I-\sum_{l \neq i} u_{l} u_{l}^{*}\right)^{-1} u_{i}}
$$

where

$$
\psi_{i, k}=\left[\left(z I-\sum_{l \neq i} u_{l} u_{l}^{*}\right)^{-1} u_{i} u_{i}^{*}\left(z I-\sum_{l \neq i} u_{l} u_{l}^{*}\right)^{-1}\right]_{k k} .
$$


Noticing that

$$
\psi_{i, k}=\frac{1}{p} x_{i}^{*} U^{*} D^{\frac{1}{2}}\left(z I-\sum_{l \neq i} u_{l} u_{l}^{*}\right)^{-1} E_{k k}\left(z I-\sum_{l \neq i} u_{l} u_{l}^{*}\right)^{-1} D^{\frac{1}{2}} U x_{i},
$$

we have by Proposition 8.1 that

$$
\begin{aligned}
& \left\|\psi_{i, k}-\frac{1}{p} \operatorname{Tr} D\left(z I-\sum_{l \neq i} u_{l} u_{l}^{*}\right)^{-1} E_{k k}\left(z I-\sum_{l \neq i} u_{l} u_{l}^{*}\right)^{-1}\right\|_{L^{2}} \\
\leq & \frac{K}{p}\left\{\operatorname{Tr} D\left(z I-\sum_{l \neq i} u_{l} u_{l}^{*}\right)^{-1} E_{k k}\left(z I-\sum_{l \neq i} u_{l} u_{l}^{*}\right)^{-1}\left(\bar{z} I-\sum_{l \neq i} u_{l} u_{l}^{*}\right)^{-1} E_{k k}\left(\bar{z} I-\sum_{l \neq i} u_{l} u_{l}^{*}\right)^{-1} D\right\}^{\frac{1}{2}} \\
\leq & \frac{C K}{p|\Im z|^{2}} .
\end{aligned}
$$

Using also Lemma 7.3, we readily have that

$$
\begin{aligned}
& \left|\mathbb{E}\left(\tilde{G}_{k k}^{S}(z)\right)-\mathbb{E}\left(\left[\left(z I-\sum_{l \neq i} u_{l} u_{l}^{*}\right)^{-1}\right]_{k k}\right)\right| \\
& \leq \frac{|z|}{p|\Im z|}\left\{\frac{C K}{|\Im z|^{2}}+\mathbb{E}\left(\left|\operatorname{Tr} D\left(z I-\sum_{l \neq i} u_{l} u_{l}^{*}\right)^{-1} E_{k k}\left(z I-\sum_{l \neq i} u_{l} u_{l}^{*}\right)^{-1}\right|\right)\right\} .
\end{aligned}
$$

Since

$$
\left|\operatorname{Tr} D\left(z I-\sum_{l \neq i} u_{l} u_{l}^{*}\right)^{-1} E_{k k}\left(z I-\sum_{l \neq i} u_{l} u_{l}^{*}\right)^{-1}\right| \leq \frac{C}{|\Im z|^{2}},
$$

Lemma 7.6 readily follows.

Hence (7.18) and Lemma 7.6 yield

$$
\mathbb{E}\left(\Phi_{i, k}\right)=\gamma_{k} \mathbb{E}\left(\tilde{G}_{k k}^{S}(z)\right)+\tau_{i, k}
$$

with $\left|\tau_{i, k}\right| \leq \frac{1}{p}(|z|+1) P\left(|\Im z|^{-1}\right)$ and thus, using equation (7.17), there exists a polynomial $Q$ with nonnegative coefficients and a sequence of nonnegative numbers $a_{N}$ converging towards zero when $N$ goes to infinity such that for any $k=1, \ldots, N$,

$$
\mathbb{E}\left(\tilde{G}_{k k}^{S}(z)\right)=\frac{1}{z}+\gamma_{k} \mathbb{E}\left(\tilde{G}_{k k}^{S}(z)\right) \mathbb{E}\left(\operatorname{tr}_{p} G_{\underline{M}_{N}^{S}}(z)\right)+\xi_{k}
$$

with

$$
\left|\xi_{k}\right| \leq(|z|+1)^{2} Q\left(|\Im z|^{-1}\right) a_{N}
$$

Thus

$$
\left\{z-\gamma_{k} z \mathbb{E}\left(\operatorname{tr}_{p} G_{\underline{M}_{N}^{S}}(z)\right)\right\} \mathbb{E}\left(\tilde{G}_{k k}^{S}(z)\right)=1+z \xi_{k}
$$


Using the resolvent identity

$$
z G_{\underline{M}_{N}^{S}}(z)=I_{p}+\underline{M}_{N}^{S} G_{\underline{M}_{N}^{S}}(z)
$$

we can easily see that

$$
\Im\left[z \operatorname{tr}_{p} G_{\underline{M}_{N}^{S}}(z)\right]=-(\Im z) \operatorname{tr}_{p} G_{\underline{M}_{N}^{S}}(z)^{*} \underline{M}_{N}^{S} G_{\underline{M}_{N}^{S}}(z) .
$$

Hence

$$
\begin{aligned}
\left|\Im\left\{z-\gamma_{k} z \mathbb{E}\left(\operatorname{tr}_{p} G_{\underline{M}_{N}^{S}}(z)\right)\right\}\right| \\
\quad=|\Im z|\left\{1+\gamma_{k} \operatorname{tr}_{p} G_{\underline{M}_{N}^{S}}(z)^{*} \underline{M}_{N}^{S} G_{\underline{M}_{N}^{S}}(z)\right\} \geq|\Im z| .
\end{aligned}
$$

Thus (17.19) yields that

$$
\mathbb{E}\left(\tilde{G}_{k k}^{S}(z)\right)=\frac{1}{z-\gamma_{k} z \mathbb{E}\left(\operatorname{tr}_{p} G_{\underline{M}_{N}^{S}}(z)\right)}+\xi_{k}^{\prime}
$$

with

$$
\left|\xi_{k}^{\prime}\right| \leq \frac{|z|}{|\Im z|}(|z|+1)^{2} Q\left(|\Im z|^{-1}\right) a_{N} .
$$

Proposition 7.1 readily follows since (see(7.14)) we have

$$
\mathbb{E}\left(\operatorname{tr}_{p} G_{\underline{M}_{N}^{S}}(z)\right)=\frac{N}{p} \mathbb{E}\left(\operatorname{tr}_{N} G_{M_{N}^{S}}(z)\right)+\frac{1-\frac{N}{p}}{z} .
$$

To prove Proposition 6.3 in the previous section, we need the following description, when the matrix $A_{N}$ and the measure $\nu$ satisfied Assumption A in the Introduction, of the convergence of $g_{\mu_{A_{N}}}(z)$ towards $g_{\nu}(z)$ and of the convergence of $\mathcal{Z}_{\frac{N}{p}, \mu_{A_{N}}}(z)$ towards $\mathcal{Z}(z)$ (dealing in the last case with measures on $\left[0 ;+\infty[)\right.$ where $\mathcal{Z}_{\frac{N}{p}, \mu_{A_{N}}}(z)$ is defined by (3.12) replacing $\nu$ by $\mu_{A_{N}}$ and $c$ by $\frac{N}{p}$.

Lemma 7.7. Under Assumption A, there exists polynomials $P_{1}$ and $P_{2}$ with nonnegative coefficients and sequences $v_{N}(1)$ and $v_{N}(2)$ of positive numbers converging towards zero such that for all $z \in \mathbb{C} \backslash \mathbb{R}$,

$$
\begin{gathered}
\left|g_{\mu_{A_{N}}}(z)-g_{\nu}(z)\right| \leq P_{1}\left(|\Im z|^{-1}\right) v_{N}(1), \\
\left|\mathcal{Z}_{\frac{N}{p}, \mu_{A_{N}}}(z)-\mathcal{Z}(z)\right| \leq(|z|+1)^{2} P_{2}\left(|\Im z|^{-1}\right) v_{N}(2),
\end{gathered}
$$

Proof: Let us introduce

$$
\hat{\nu}_{N}=\frac{1}{N-r} \sum_{j=1}^{N-r} \delta_{\beta_{j}(N)} .
$$


Let us fix $\epsilon>0$. According to the assumption (2.1), for $N$ large all the $\beta_{j}(N)$ are in the set $\{x, d(x, \operatorname{supp} \nu)<\epsilon\}$. Moreover, $\{x, d(x, \operatorname{supp} \nu)<\epsilon\}$ may be covered by a finite number $n_{\epsilon}$ of disjoint intervals $I_{i}(\epsilon)$ with diameter smaller than $\epsilon$, of the form $\left.] a_{i}(\epsilon) ; b_{i}(\epsilon)\right]$ where $a_{i}(\epsilon)$ and $b_{i}(\epsilon)$ are two continuity points of the distribution function of $\nu$. Note that for any $i=1, \ldots, n_{\epsilon}$, when $N$ goes to infinity,

$$
\hat{\nu}_{N}\left(I_{i}(\epsilon)\right) \rightarrow \nu\left(I_{i}(\epsilon)\right) .
$$

Since $\left|\frac{1}{N} \sum_{i=1}^{J} \frac{1}{z-\theta_{i}}\right| \leq \frac{r}{N}|\Im z|^{-1}$, and $\left|\left[\frac{1}{N}-\frac{1}{N-r}\right] \sum_{j=1}^{N-r} \frac{1}{z-\beta_{j}(N)}\right| \leq \frac{r}{N}|\Im z|^{-1}$, we focus on the difference $g_{\hat{\nu}_{N}}(z)-g_{\nu}(z)$. Similarly, since $\left|\frac{1}{N} \sum_{i=1}^{J} \frac{\theta_{i}}{1-\theta_{i} z}\right| \leq$ $\frac{r}{N}|\Im z|^{-1}$, and $\left|\left[\frac{1}{N}-\frac{1}{N-r}\right] \sum_{j=1}^{N-r} \frac{\beta_{j}(N)}{1-\beta_{j}(N) z}\right| \leq \frac{r}{N}|\Im z|^{-1}$, we focus on the difference $\mathcal{Z}_{\frac{N}{p}, \hat{\nu}_{N}}(z)-\mathcal{Z}(z)$ where $\mathcal{Z}_{\frac{N}{p}, \hat{\nu}_{N}}$ is defined by (3.12) replacing $\nu$ by $\hat{\nu}_{N}$ and $c$ by $\frac{N}{p}$.

$$
\begin{aligned}
g_{\hat{\nu}_{N}}(z)-g_{\nu}(z) & =\sum_{i=1}^{n_{\epsilon}}\left\{\frac{1}{N-r} \sum_{\beta_{j}(N) \in I_{i}(\epsilon)} \frac{1}{z-\beta_{j}(N)}-\int_{I_{i}(\epsilon)} \frac{1}{z-x} d \nu(x)\right\} \\
& =\sum_{i, \nu\left(I_{i}(\epsilon)\right)=0} \frac{1}{N-r} \sum_{\beta_{j}(N) \in I_{i}(\epsilon)} \frac{1}{z-\beta_{j}(N)} \\
& +\sum_{i, \nu\left(I_{i}(\epsilon)\right)>0} \frac{1}{N-r} \sum_{\beta_{j}(N) \in I_{i}(\epsilon)} \frac{1}{\nu\left(I_{i}(\epsilon)\right)} \int_{I_{i}(\epsilon)}\left(\frac{1}{z-\beta_{j}(N)}-\frac{1}{z-x}\right) d \nu(x) \\
& +\sum_{i, \nu\left(I_{i}(\epsilon)\right)>0}\left(\frac{\hat{\nu}_{N}\left(I_{i}(\epsilon)\right)}{\nu\left(I_{i}(\epsilon)\right)}-1\right) \int_{I_{i}(\epsilon)} \frac{1}{z-x} d \nu(x) \\
& =\Delta_{1}+\Delta_{2}+\Delta_{3} .
\end{aligned}
$$

where

$$
\begin{gathered}
\left|\Delta_{1}\right| \leq \sum_{i, \nu\left(I_{i}(\epsilon)\right)=0} \hat{\nu}_{N}\left(I_{i}(\epsilon)\right)|\Im z|^{-1}, \\
\left|\Delta_{2}\right| \leq \epsilon \sum_{i, \nu\left(I_{i}(\epsilon)\right)>0} \hat{\nu}_{N}\left(I_{i}(\epsilon)\right)|\Im z|^{-2} \leq \epsilon|\Im z|^{-2}, \\
\left|\Delta_{3}\right| \leq \sum_{i, \nu\left(I_{i}(\epsilon)\right)>0}\left|\hat{\nu}_{N}\left(I_{i}(\epsilon)\right)-\nu\left(I_{i}(\epsilon)\right)\right||\Im z|^{-1} .
\end{gathered}
$$

Hence

$$
\left|g_{\hat{\nu}_{N}}(z)-g_{\nu}(z)\right| \leq\left(|\Im z|^{-2}+|\Im z|^{-1}\right)\left(\epsilon+\sum_{i=1}^{n_{\epsilon}}\left|\hat{\nu}_{N}\left(I_{i}(\epsilon)\right)-\nu\left(I_{i}(\epsilon)\right)\right|\right)
$$

and then

$$
\limsup _{N \rightarrow+\infty} \sup _{z \in \mathbb{C} \backslash \mathbb{R}}\left\{\left(|\Im z|^{-2}+|\Im z|^{-1}\right)^{-1}\left|g_{\hat{\nu}_{N}}(z)-g_{\nu}(z)\right|\right\} \leq \epsilon .
$$


Since this is true for any $\epsilon>0$, we get that

$$
\lim _{N \rightarrow+\infty} \sup _{z \in \mathbb{C} \backslash \mathbb{R}}\left\{\left(|\Im z|^{-2}+|\Im z|^{-1}\right)^{-1}\left|g_{\hat{\nu}_{N}}(z)-g_{\nu}(z)\right|\right\}=0
$$

which yields (7.21).

Now, since moreover $\left|\mathcal{Z}_{\frac{N}{p}, \hat{\nu}_{N}}(z)-\mathcal{Z}_{c, \hat{\nu}_{N}}(z)\right| \leq\left|\frac{N}{p}-c\right||\Im z|^{-1}$ we will study $\mathcal{Z}_{c, \hat{\nu}_{N}}(z)-\mathcal{Z}(z)$. Similarly,

$$
\begin{aligned}
\frac{1}{c}\left[\mathcal{Z}_{c, \hat{\nu}_{N}}(z)-\mathcal{Z}(z)\right] & =\sum_{i=1}^{n_{\epsilon}}\left\{\frac{1}{N-r} \sum_{\beta_{j}(N) \in I_{i}(\epsilon)} \frac{\beta_{j}(N)}{1-\beta_{j}(N) z}-\int_{I_{i}(\epsilon)} \frac{x}{1-x z} d \nu(x)\right\} \\
& =\sum_{i, \nu\left(I_{i}(\epsilon)\right)=0} \frac{1}{N-r} \sum_{\beta_{j}(N) \in I_{i}(\epsilon)} \frac{\beta_{j}(N)}{1-\beta_{j}(N) z} \\
& +\sum_{i, \nu\left(I_{i}(\epsilon)\right)>0} \frac{1}{N-r} \sum_{\beta_{j}(N) \in I_{i}(\epsilon)} \frac{1}{\nu\left(I_{i}(\epsilon)\right)} \int_{I_{i}(\epsilon)}\left(\frac{\beta_{j}(N)}{1-\beta_{j}(N) z}-\frac{x}{1-x z}\right) d \nu(x) \\
& +\sum_{i, \nu\left(I_{i}(\epsilon)\right)>0}\left(\frac{\hat{\nu}_{N}\left(I_{i}(\epsilon)\right)}{\nu\left(I_{i}(\epsilon)\right)}-1\right) \int_{I_{i}(\epsilon)} \frac{x}{1-x z} d \nu(x) \\
& =\Delta_{1}+\Delta_{2}+\Delta_{3} .
\end{aligned}
$$

where

$$
\left|\Delta_{1}\right| \leq \sum_{i, \nu\left(I_{i}(\epsilon)\right)=0} \hat{\nu}_{N}\left(I_{i}(\epsilon)\right)|\Im z|^{-1}
$$

$$
\begin{aligned}
&\left|\Delta_{2}\right| \leq \sum_{i, \nu\left(I_{i}(\epsilon)\right)>0}\left\{\frac{1}{N-r} \sum_{\beta_{j}(N) \in I_{i}(\epsilon)} \frac{1}{\nu\left(I_{i}(\epsilon)\right)} \int_{I_{i}(\epsilon)} \frac{\left|\beta_{j}(N)-x\right|}{|z|^{2}\left(\left|\frac{1}{z}-\beta_{j}(N)\right|\left|\frac{1}{z}-x\right|\right.} d \nu(x)\right\} \\
& \leq \epsilon \sum_{i, \nu\left(I_{i}(\epsilon)\right)>0} \hat{\nu}_{N}\left(I_{i}(\epsilon)\right)|z|^{-2}\left|\Im\left(\frac{1}{z}\right)\right|^{-2} \\
& \leq \epsilon|z|^{2}|\Im z|^{-2}, \\
&\left|\Delta_{3}\right| \leq \sum_{i, \nu\left(I_{i}(\epsilon)\right)>0}\left|\hat{\nu}_{N}\left(I_{i}(\epsilon)\right)-\nu\left(I_{i}(\epsilon)\right)\right||\Im z|^{-1} .
\end{aligned}
$$

Hence

$$
\left|\frac{1}{c}\left[\mathcal{Z}_{c, \hat{\nu}_{N}}(z)-\mathcal{Z}(z)\right]\right| \leq\left(|z|^{2}|\Im z|^{-2}+|\Im z|^{-1}\right)\left(\epsilon+\sum_{i=1}^{n_{\epsilon}}\left|\hat{\nu}_{N}\left(I_{i}(\epsilon)\right)-\nu\left(I_{i}(\epsilon)\right)\right|\right)
$$

and then

$$
\limsup _{N \rightarrow+\infty} \sup _{z \in \mathbb{C} \backslash \mathbb{R}}\left\{\left(|z|^{2}|\Im z|^{-2}+|\Im z|^{-1}\right)^{-1}\left|\frac{1}{c}\left[\mathcal{Z}_{c, \hat{\nu}_{N}}(z)-\mathcal{Z}(z)\right]\right|\right\} \leq \epsilon .
$$


Since this is true for any $\epsilon>0$, we get that

$$
\lim _{N \rightarrow+\infty} \sup _{z \in \mathbb{C} \backslash \mathbb{R}}\left\{\left(|z|^{2}|\Im z|^{-2}+|\Im z|^{-1}\right)^{-1}\left|\mathcal{Z}_{c, \hat{\nu}_{N}}(z)-\mathcal{Z}(z)\right|\right\}=0
$$

and (7.22) follows.

In the sample covariance matrix setting we will need the following upper bound of $\frac{1}{\Im\left(\underline{g}_{N}^{S}(z)\right)}$ where

$$
\underline{g}_{N}^{S}(z)=\mathbb{E}\left(\operatorname{tr}_{p} G_{\underline{M}_{N}^{S}}(z)\right)=\frac{N}{p} \mathbb{E}\left(\operatorname{tr}_{N} G_{M_{N}^{S}}(z)\right)+\frac{1-\frac{N}{p}}{z},
$$

with

$$
\underline{M}_{N}^{S}=\frac{1}{p} B_{N}^{*} A_{N} B_{N} .
$$

Lemma 7.8. There exists a constant $\underline{C}$ such that for any $z$ in $\mathbb{C} \backslash \mathbb{R}$,

$$
\left|\frac{1}{\Im\left(\underline{g}_{N}^{S}(z)\right)}\right| \leq \underline{C}(1+|z|)^{2}|\Im z|^{-1}
$$

Proof: Note that

$$
\left|\Im\left(\underline{g}_{N}^{S}(z)\right)\right|=|\Im z| \mathbb{E}\left[\int_{\mathbb{R}} \frac{d \mu_{\underline{M}_{N}}(x)}{|z-x|^{2}}\right] .
$$

Now, for any $x$ in the spectrum of $\underline{M}_{N}^{S}$,

$$
|z-x|^{2} \leq 2\left(|z|^{2}+\left\|\underline{M}_{N}^{S}\right\|^{2}\right) \leq 2\left(|z|^{2}+\left\|A_{N}\right\|^{2}\left\|\frac{1}{p} B_{N}^{*} B_{N}\right\|^{2}\right)
$$

so that, with $C=\sup _{N}\left\|A_{N}\right\|$,

$$
\mathbb{E}\left[\int_{\mathbb{R}} \frac{d \mu_{\underline{M}_{N}}(x)}{|z-x|^{2}}\right] \geq \mathbb{E}\left[\frac{1}{2\left(|z|^{2}+C^{2}\left\|\frac{1}{p} B_{N}^{*} B_{N}\right\|^{2}\right)}\right] .
$$

According to Theorem 5.11 in [7, $\left\|\frac{1}{p} B_{N}^{*} B_{N}\right\|=c\left(1+\frac{1}{\sqrt{c}}\right)^{2}+o_{a . s, N}(1)$ so that by the dominated convergence Theorem we can deduce that for all large $N$,

$$
\mathbb{E}\left[\int_{\mathbb{R}} \frac{d \mu_{\underline{M}_{N}}(x)}{|z-x|^{2}}\right] \geq \frac{1}{2\left(|z|^{2}+C_{1}\right)}
$$

where $C_{1}>\left[C c\left(1+\frac{1}{\sqrt{c}}\right)^{2}\right]^{2}$. Therefore

$$
\left|\frac{1}{\Im\left(\underline{g}_{N}^{S}(z)\right)}\right| \leq \frac{2\left(|z|^{2}+C_{1}\right)}{|\Im z|} .
$$

so that Lemma 7.8 readily follows. 


\section{Appendix}

\subsection{Poincaré inequality and concentration inequalities}

We first derive in this section concentration inequalities based on the Poincaré inequality. We refer the reader to the book [2]. A probability measure $\mu$ on $\mathbb{R}$ is said to satisfy the Poincaré inequality with constant $C_{P I}$ if for any $\mathcal{C}^{1}$ function $f: \mathbb{R} \rightarrow \mathbb{C}$ such that $f$ and $f^{\prime}$ are in $L^{2}(\mu)$,

$$
\mathbf{V}(f) \leq C_{P I} \int\left|f^{\prime}\right|^{2} d \mu,
$$

with $\mathbf{V}(f)=\int\left|f-\int f d \mu\right|^{2} d \mu$.

We refer the reader to 21] for a characterization of the measures on $\mathbb{R}$ which satisfy a Poincaré inequality.

Remark 8.1. If the law of a random variable $X$ satisfies the Poincaré inequality with constant $C_{P I}$ then, for any fixed $\alpha \neq 0$, the law of $\alpha X$ satisfies the Poincaré inequality with constant $\alpha^{2} C_{P I}$.

If a probability measure $\mu$ on $\mathbb{R}$ satisfies the Poincaré inequality with constant $C_{P I}$ then the product measure $\mu^{\otimes M}$ on $\mathbb{R}^{M}$ satisfies the Poincaré inequality with constant $C_{P I}$ in the sense that for any differentiable function $F$ such that $F$ and its gradient $\nabla F$ are in $L^{2}\left(\mu^{\otimes M}\right)$,

$$
\mathbf{V}(f) \leq C_{P I} \int\|\nabla F\|_{2}^{2} d \mu^{\otimes M}
$$

with $\mathbf{V}(f)=\int\left|f-\int f d \mu^{\otimes M}\right|^{2} d \mu^{\otimes M}$ (see Theorem 2.5 in [33]).

An important consequence of the Poincaré inequality is the following concentration result.

Lemma 8.1. Lemma 4.4.3 and Exercise 4.4 .5 in [1] or Chapter 3 in [41]. Let $\mathbb{P}$ be a probability measure on $\mathbb{R}^{\mathbb{M}}$ which satisfies a Poincaré inequality with constant $C_{P I}$. Then there exists $K_{1}>0$ and $K_{2}>0$ such that, for any Lipschitz function $F$ on $\mathbb{R}^{M}$ with Lipschitz constant $|F|_{\text {Lip }}$,

$$
\forall \epsilon>0, \mathbb{P}\left(\left|F-\mathbb{E}_{\mathbb{P}}(F)\right|>\epsilon\right) \leq K_{1} \exp \left(-\frac{\epsilon}{K_{2} \sqrt{C_{P I}}|F|_{\text {Lip }}}\right) .
$$

\subsection{Technical tools}

We need the following result on the extension of Lipschitz functions on $\mathbb{R}$ to the Hermitian matrices.

Lemma 8.2. (see [27]) Let $f$ be a real $C_{\mathcal{L}}$-Lipschitz function on $\mathbb{R}$. Then its extension on the $N \times N$ Hermitian matrices is $C_{\mathcal{L}}$-Lipschitz with respect to the norm $\|M\|_{2}=\left\{\operatorname{Tr}\left(M M^{*}\right)\right\}^{\frac{1}{2}}$. 
Proof: Let $A$ and $B$ be $N \times N$ Hermitian matrices. Let us consider their spectral decompositions

$$
A=\sum_{i} \lambda_{i}(A) P_{i}^{(A)}
$$

and

$$
B=\sum_{i} \lambda_{i}(B) P_{i}^{(B)}
$$

We have

$$
\begin{aligned}
\|f(B)-f(A)\|_{2}^{2}= & \operatorname{Tr}\left(\sum_{i} f\left(\lambda_{i}(A)\right) P_{i}^{(A)}-\sum_{i} f\left(\lambda_{i}(B)\right) P_{i}^{(B)}\right)^{2} \\
= & \operatorname{Tr}\left(\sum_{i} f\left(\lambda_{i}(A)\right)^{2} P_{i}^{(A)}+\sum_{j} f\left(\lambda_{j}(B)\right)^{2} P_{j}^{(B)}\right) \\
& -2 \sum_{i, j} f\left(\lambda_{i}(A)\right) f\left(\lambda_{j}(B)\right) \operatorname{Tr}\left(P_{i}^{(A)} P_{j}^{(B)}\right) \\
= & \operatorname{Tr}\left(\sum _ { i j } \left(f\left(\lambda_{i}(A)\right)^{2} P_{i}^{(A)} P_{j}^{(B)}+\sum_{i, j}\left(f\left(\lambda_{j}(B)\right)^{2} P_{i}^{(A)} P_{j}^{(B)}\right)\right.\right. \\
& -2 \sum_{i, j} f\left(\lambda_{i}(A)\right) f\left(\lambda_{j}(B)\right) \operatorname{Tr}\left(P_{i}^{(A)} P_{j}^{(B)}\right) \\
= & \sum_{i, j}\left(f\left(\lambda_{i}(A)\right)-f\left(\lambda_{j}(B)\right)^{2} \operatorname{Tr}\left(P_{i}^{(A)} P_{j}^{(B)}\right) .\right.
\end{aligned}
$$

Now, since $\operatorname{Tr}\left(P_{i}^{(A)} P_{j}^{(B)}\right) \geq 0$, we can deduce that

$$
\|f(B)-f(A)\|_{2}^{2} \leq \sum_{i, j} C_{\mathcal{L}}^{2}\left(\lambda_{i}(A)-\lambda_{j}(B)\right)^{2} \operatorname{Tr}\left(P_{i}^{(A)} P_{j}^{(B)}\right)=C_{\mathcal{L}}^{2}\|B-A\|_{2}^{2} . \square
$$

We recall here some useful properties of the resolvent (see [39, 22]).

Lemma 8.3. For a $N \times N$ Hermitian or symmetric matrix $M$, for any $z \in$ $\mathbb{C} \backslash \operatorname{Spect}(M)$, we denote by $G(z):=\left(z I_{N}-M\right)^{-1}$ the resolvent of $M$.

Let $z \in \mathbb{C} \backslash \mathbb{R}$,

(i) $\|G(z)\| \leq|\Im z|^{-1}$ where $\|$.$\| denotes the operator norm.$

(ii) $\left|G(z)_{i j}\right| \leq|\Im z|^{-1}$ for all $i, j=1, \ldots, N$.

(iii) Let $z \in \mathbb{C}$ such that $|z|>\|M\|$; we have

$$
\|G(z)\| \leq \frac{1}{|z|-\|M\|}
$$

We recall here the following classical result due to Weyl. 
Lemma 8.4. (cf. Theorem 4.3.7 of [37]) Let $B$ and $C$ be two $N \times N$ Hermitian matrices. For any pair of integers $j, k$ such that $1 \leq j, k \leq N$ and $j+k \leq N+1$, we have

$$
\lambda_{j+k-1}(B+C) \leq \lambda_{j}(B)+\lambda_{k}(C) .
$$

For any pair of integers $j, k$ such that $1 \leq j, k \leq N$ and $j+k \geq N+1$, we have

$$
\lambda_{j}(B)+\lambda_{k}(C) \leq \lambda_{j+k-N}(B+C) .
$$

The following result on quadratic forms is of basic use in the sample covariance matrix setting. Note that, a complex random variable $x$ will be said standardized if $\mathbb{E}(x)=0$ and $\mathbb{E}\left(|x|^{2}\right)=1$.

Proposition 8.1. (Lemma 2.7 [5]) Let $B=\left(b_{i j}\right)$ be a $N \times N$ matrix and $Y_{N}$ be a vector of size $N$ which contains i.i.d standardized entries with bounded fourth moment. Then there is a constant $K>0$ such that

$$
\mathbb{E}\left|Y_{N}^{*} B Y_{N}-\operatorname{Tr} B\right|^{2} \leq K \operatorname{Tr}\left(B B^{*}\right) .
$$

The following technical lemma is fundamental in this paper. We refer the reader to the Appendix of [22] where it is proved using the ideas of [35].

Lemma 8.5. Let $h$ be an analytic function on $\mathbb{C} \backslash \mathbb{R}$ which satisfies

$$
|h(z)| \leq(|z|+K)^{\alpha} P\left(|\Im z|^{-1}\right)
$$

and $\varphi$ be in $\mathcal{C}^{\infty}(\mathbb{R}, \mathbb{R})$ with compact support. Then,

$$
\limsup _{y \rightarrow 0^{+}}\left|\int_{\mathbb{R}} \varphi(x) h(x+i y) d x\right|<+\infty .
$$

Acknowledgments: I am grateful to Charles Bordenave for useful discussions. I would like to thank the anonymous referees for their careful reading and their pertinent comments which led to an overall improvement of the paper.

\section{References}

[1] G. Anderson, A. Guionnet, and O. Zeitouni. An Introduction to Random Matrices. Cambridge University Press, 2009.

[2] Ané C., Blachère S., Chafaї D., Fougères P., Gentil I., MalRieu F., Roberto C., Scheffer G.. Sur les inégalités de Sobolev logarithmiques (French) [Logarithmic Sobolev inequalities], Panoramas et Synthèses [Panoramas and Syntheses] 10, S.M.F Paris, 2000.

[3] Z. D. Bai. Methodologies in spectral analysis of large-dimensional random matrices, a review. Statist. Sinica, 9(3):611-677, 1999. With comments by G. J. Rodgers and Jack W. Silverstein; and a rejoinder by the author. 
[4] Z. D. Bai and J. W. Silverstein. On the empirical distribution of eigenvalues of a class of large dimensional random matrices. J. Multivariate Anal., 54:175-192, 1995.

[5] Z. D. Bai and J. W. Silverstein. No eigenvalues outside the support of the limiting spectral distribution of large-dimensional sample covariance matrices. Ann. Probab., 26(1):316-345, 1998.

[6] Z. D. Bai and J. W. Silverstein. Exact separation of eigenvalues of largedimensional sample covariance matrices. Ann. Probab., 27(3):1536-1555, 1999.

[7] Z. D. Bai and J. W. Silverstein. Spectral Analysis of of large-dimensional random matrices. Mathematics Monograph Series 2, Science Press Beijing 2006.

[8] Z. D. Bai, J. W. Silverstein and Y.Q. Yin. A note on the limit of the largest eigenvalue of a large-dimensional sample covariance matrix. J. Multivariate Anal., 26(2):166-168, 1988.

[9] Z. D. Bai and J. Yao. Limit theorems for sample eigenvalues in a generalized spiked population model. ArXiv e-prints, June 2008.

[10] Z. D. Bai and Y. Q. Yin. Necessary and sufficient conditions for almost sure convergence of the largest eigenvalue of a Wigner matrix. Ann. Probab., 16(4):1729-1741, 1988.

[11] J. Baik, G. Ben Arous, and S. Péché. Phase transition of the largest eigenvalue for nonnull complex sample covariance matrices. Ann. Probab., 33(5):1643-1697, 2005.

[12] J. Baik and J. W. Silverstein. Eigenvalues of large sample covariance matrices of spiked population models. J. Multivariate Anal., 97(6):1382-1408, 2006.

[13] S. T. Belinschi and H. Bercovici. Partially defined semigroups relative to multiplicative free convolution. Int. Math. Res. Not., (2):65-101, 2005.

[14] S. T. Belinschi and H. Bercovici. A new approach to subordination results in free probability. J. Anal. Math., 101:357-365, 2007.

[15] F. Benaych-Georges and R. N. Rao. The eigenvalues and eigenvectors of finite, low rank perturbations of large random matrices. Adv. in Math., 227(1):494-521, 2011.

[16] F. Benaych-Georges and R. N. Rao. The singular values and vectors of low rank perturbations of large rectangular random matrices. ArXiv e-prints: 1103.2221, 2011.

[17] H. Bercovici and D. Voiculescu. Free convolution of measures with unbounded support. Indiana Univ. Math. J., 42(3):733-773, 1993. 
[18] P. Biane. On the free convolution with a semi-circular distribution. Indiana Univ. Math. J., 46(3):705-718, 1997.

[19] P. Biane. Processes with free increments. Math. Z., 227(1):143-174, 1998.

[20] P. Biane. Free Probability for Probabilists. Quantum probability communications, Vol. XI (Grenoble, 1998), QP-PQ, XI, World Sci. Publishing, River Edge,NJ: 55-71, 2003.

[21] S. G. Bobkov and F. Götze. Exponential integrability and transportation cost related to logarithmic Sobolev inequalities. J. Funct. Anal., 163(1):128, 1999.

[22] M. Capitaine and C. Donati-Martin. Strong asymptotic freeness for Wigner and Wishart matrices. Indiana Univ. Math. J., 56(2):767-803, 2007.

[23] M. Capitaine, C. Donati-Martin, and D. Féral. The largest eigenvalues of finite rank deformation of large Wigner matrices: convergence and nonuniversality of the fluctuations. Ann. Probab., 37(1):1-47, 2009.

[24] M. Capitaine, C. Donati-Martin, D. Féral and M. Février. Free convolution with a semi-circular distribution and eigenvalues of spiked deformations of Wigner matrices. Electronic Journal of Probability, 16: 1750-1792, 2011.

[25] S. Choi and J.W. Silverstein. Analysis of the limiting spectral distribution of large dimensional random matrices. J. Multivariate. Anal., 54: 295-309, 1995.

[26] K. Dykema. On certain free product factors via an extended matrix model. J. Funct. Anal., 112(1):31-60, 1993.

[27] Y. Doumerc. Quelques aspects du spectre des grandes matrices aléatoires. Mémoire de D.E.A.

[28] S.C. Eisenstat and I.C.F. Ipsen. Relative perturbation results for eigenvalues and eigenvectors of diagonalisable matrices BIT, 38(3):502-509, 1998.

[29] D. Féral and S. Péché. The largest eigenvalue of rank one deformation of large Wigner matrices. Comm. Math. Phys., 272(1):185-228, 2007.

[30] Z. Füredi and J. Komlós. The eigenvalues of random symmetric matrices. Combinatorica, 1(3):233-241, 1981.

[31] S. Geman. A limit theorem for the norm of random matrices. Ann. Probab., 8 (2): 252261, 1980.

[32] U. Grenander and J. W. Silverstein. Spectral analysis of networks with random topologies. SIAM J. Appl. Math., 32:499-519, 1977.

[33] A. Guionnet and B. Zegarlinski. Lectures on Logarithmic Sobolev inequalities. In Séminaire de Probabilités, XXXVI, volume 1801 of Lecture Notes in Math.. Springer, Berlin, 2003. 
[34] A. Guionnet and O. Zeitouni. Concentration of the spectral measure for large matrices. Electronic Communications in Probability,5: 119-136, 2000.

[35] U. Haagerup and S. Thorbjørnsen. A new application of random matrices: $\operatorname{Ext}\left(C_{\mathrm{red}}^{*}\left(F_{2}\right)\right)$ is not a group. Ann. of Math. (2), 162(2):711-775, 2005.

[36] F. Hiai and D. Petz. The Semicircle Law, Free Random Variables and Entropy Mathematical Surveys and Monographs Volume 77, American Mathematical Society.

[37] R. A. Horn and C. R. Johnson. Matrix analysis. Cambridge University Press, Cambridge, 1990. Corrected reprint of the 1985 original.

[38] I. Johnstone. On the distribution of the largest eigenvalue in principal components analysis. Ann. Stat. , 29:295-327, 2001.

[39] A. M. Khorunzhy, B. A. Khoruzhenko, and L. A. Pastur. Asymptotic properties of large random matrices with independent entries. J. Math. Phys., 37(10):5033-5060, 1996.

[40] P.R. Krishnaiah and Y. Q. Yin. A limit theorem for the eigenvalues of product of two random matrices. J. Multivariate Anal., 13:489-507, 1983.

[41] M. Ledoux. The concentration of Measure Phenomenon. American Mathematical Society, Providence, RI, 2001.

[42] A. Martchenko and L. Pastur. Distribution of eigenvalues for some sets of random matrices. Math. USSR-Sb. 1: 457-486, 1967.

[43] J. Mingo and R. Speicher. Free probability and Random matrices. Personal Communication, 2010.

[44] D. Paul. Asymptotics of sample eigenstructure for a large dimensional spiked covariance model Statist. Sinica, 17 (4):1617-1642, 2007.

[45] S. Péché. Non-white Wishart ensembles. J. Multivariate Anal., 97(4):874894, 2006.

[46] S. Péché and O. Ledoit. Eigenvectors of some large sample covariance matrix ensembles. Probab. Theory Relat. Fields, online 2010.

[47] N. R. Rao and J. W. Silverstein. Fundamental limit of sample generalized eigenvalue based detection of signals in noise using relatively few signalbearing and noise-only samples. IEEE Journal of Selected Topics in Signal Processing, 4(3): 468-480, 2010.

[48] J. W. Silverstein. Strong convergence of the empirical distribution of eigenvalues of large dimensional random matrices. J. Multivariate Anal., 55(4):331-339, 1995. 
[49] D. Voiculescu. Addition of Certain Non commuting Random Variables $J$. Funct. Anal., 66:323-346, 1986.

[50] D. Voiculescu. Multiplication of Certain Non commuting Random Variables Journal of Operator Theory, 18:223-235, 1987.

[51] D. Voiculescu. Limit laws for random matrices and free products. Invent. Math., 104(1):201-220, 1991.

[52] D. Voiculescu. The analogues of entropy and of Fisher's information measure in free probability theory. I. Comm. Math. Phys., 155(1):71-92, 1993.

[53] D. V. Voiculescu, K. J. Dykema, and A. Nica. Free random variables, volume 1 of CRM Monograph Series. American Mathematical Society, Providence, RI, 1992. A noncommutative probability approach to free products with applications to random matrices, operator algebras and harmonic analysis on free groups.

[54] K.W. Wachter. The strong limits of random matrix spectra for sample matrices of independent elements. Ann. Probab. ,6:1-18, 1978.

[55] E. P. Wigner. Characteristic vectors of bordered matrices with infinite dimensions. Ann. of Math. (2), 62:548-564, 1955.

[56] E. P. Wigner. On the distribution of the roots of certain symmetric matrices. Ann. of Math. (2), 67:325-327, 1958.

[57] Y.Q. Yin. Limiting spectral distribution for a class of random matrices $J$. Multivariate Anal., 20: 50-68, 1986.

[58] Y.Q. Yin, Z.D. Bai and P.R. Krishnaiah. On the limit of the largest eigenvalue of the large-dimensional sample covariance matrix. Probab. Theory Relared Fields , 78 (4):509-521, 1988. 